Estudo dos retratos de fase dos campos de vetores polinomiais quadráticos com integral primeira racional de grau 2 
SERVIÇO DE PÓS-GRADUAÇÃO DO ICMC-USP

Dada de Depósito:

Assinatura:

\title{
Estudo dos retratos de fase dos campos de vetores polinomiais quadráticos com integral primeira racional de grau 2
}

\author{
Daniela Peruzzi \\ Orientadora: Profa. Dra. Regilene Delazari dos Santos Oliveira
}

Dissertação apresentada ao Instituto de Ciências Matemáticas e de Computação - ICMC-USP, como parte dos requisitos para obtenção do título de Mestre em Matemática.

USP - São Carlos

Maio/2009 
- minha màe, exempla de sabedoria

e vigor e a quem devo tudo a que sou.

- memória de minha quevida amiga OPlly, exempla de forca e determinacão. 



\section{Agradecimentos}

Agradeço a Deus que me permitiu essa e muitas outras conquistas, ao longo de toda a minha vida, e não somente nestes anos como universitária.

Agradeço à minha mãe, Maria, pelo amor incondicional, pela dedicação, pelo respeito, pela confiança que depositou em mim, pelo incentivo, pelos conselhos, por ter me segurado pelas mãos e me levantado de todas as quedas e por nunca ter me deixado desistir. Pelo exemplo de coragem, humildade, força e determinação. Mãe, você é meu maior exemplo de vida!

Agradeço ao meu pai, José, pelo amor, pela paciência, pelos ensinamentos, pela compreensão. Pelo exemplo de superação e de humildade.

Agradeço ao meu irmão, Danilo, pela amizade, pelo apoio e por ter sempre acreditado em mim. Você é o melhor irmão do mundo!

Agradeço a toda minha família (avós, tios, tias, primos e primas), que nos momentos de minha ausência dedicados ao estudo, sempre souberam entender que o futuro é feito a partir da constante dedicação no presente. Obrigada pelo apoio incondicional que sempre dispensaram em todas as minhas realizações.

Agradeço ao Bruno, pelo incentivo, pelo carinho, paciência e compreensão.

Agradeço a todos os meus amigos, em especial, à Polly, grande amiga e companheira de estudos durante o ensino médio, a graduação e iniciação científica. Por estar sempre ao meu lado, nos momentos difíceis e nos momentos de diversão. Pelas longas conversas, baladas, pelos segredos confidenciados, pelos conselhos. Por todas as conquistas que tivemos juntas. Por me ouvir e me incentivar. Por ter me ensinado a viver com intensidade e alegria cada momento de minha vida. Você estará para sempre viva em meu coração.

Agradeço também aos amigos de pós-graduação, em especial, aos companheiros da sala 4-007 com quem dividi grande parte de minhas horas de estudo. Pelo apoio, pelos 
momentos de aprendizagem e descontração.

Agradeço a professora Regilene, pela amizade, pela confiança, pela dedicação, por todos os ensinamentos ao longo de todos esses anos de orientação, não só de mestrado, mas também de iniciação científica, por me encorajar nas horas difíceis e por me apoiar em todas as minhas decisões. Obrigada por ter acreditado em meu potencial e por alimentar em mim um amor cada vez maior pela matemática.

Agradeço a todos os professores do ICMC que fizeram parte de minha vida acadêmica.

Agradeço a todos os funcionários do ICMC, pela atenção.

Agradeço à CAPES e ao CNPq pelo suporte financeiro.

Enfim, a todos que de alguma forma, contribuíram para essa conquista, meu mais sincero: muito obrigada! 
"A percepção do desconhecido é a mais fascinante das experiências. O homem que não tem os olhos abertos para o misterioso passará pela vida sem ver nada."

(Albert Einstein) 



\section{Resumo}

Um dos principais problemas na teoria qualitativa das equações diferenciais em dimensão dois é apresentar, para uma dada família de sistemas diferenciais, uma classificação topológica dos retratos de fase de todos os sistemas dessa família.

A proposta deste trabalho é estudar a técnica utilizada na classificação dos retratos de fase globais de sistemas diferenciais polinomiais da forma

$$
\frac{d x}{d t}=P(x, y) \quad \frac{d y}{d t}=Q(x, y)
$$

onde $P$ e $Q$ são polinômios nas variáveis $x$ e $y$ e o máximo entre os graus de $P$ e $Q$ é 2 .

Para esse fim optamos pelo estudo da referência de Cairó e Llibre [5]. Na presente referência os autores obtém a classificação de todos os retratos de fase globais dos sistemas diferenciais polinomiais que possuem uma integral primeira racional, $H$, de grau 2 .

Esse estudo foi dividido em duas etapas. Na primeira, caracterizamos a função $H$ através de seus coeficientes. Na segunda, encontramos todos os retratos de fase globais no disco de Poincaré. Para tais sistemas, existem exatamente 18 retratos de fase no disco de Poincaré, exceto pela reversão do sentido de todas as órbitas ou equivalência topológica. 



\section{Abstract}

One of the main problems in the qualitative theory of 2-dimensional differential equations is, for a concrete family of differential systems, to describe a topological classification of the phase portraits for all the systems in this family.

The purpose of this work is to study a technique used in the classification of global phase portraits of the planar polynomial differential systems or simply quadratic systems of the form

$$
\frac{d x}{d t}=P(x, y) \quad \frac{d y}{d t}=Q(x, y)
$$

where $P$ and $Q$ are real polynomials in $x$ and $y$ and the maximum degree of $P$ and $Q$ is 2 .

Our basic reference is the paper of Cairó and Llibre [5]. In that work the authors give the classification of all global phase portraits of the planar quadratic differential systems having a rational first integral $H$ of degree 2 .

Our work is divided in two parts. In the first part, we characterize the first integral $H$ through its coefficients. In the second one, we describe all global phase portraits in the Poincaré disk. For such systems, there are exactly 18 different phase portraits in the Poincaré disk, up to a reversal of sense of all orbits or topological equivalence. 



\section{Sumário}

1 Conceitos Básicos 3

1.1 Integral primeira e curvas invariantes . . . . . . . . . . . . . . . . . 3

1.2 Pontos singulares . . . . . . . . . . . . . . . . . . . . . 6

1.3 Blow up ............................... 11

1.3.1 Blow up polar . . . . . . . . . . . . . . . . . . . 11

1.3.2 Blow up direcional . . . . . . . . . . . . . . . . . . . 15

1.3.3 Relação entre blow up polar e blow up direcional . . . . . . . . . . . . 17

1.4 Compactificação de Poincaré . . . . . . . . . . . . . . . . . . 23

1.4.1 Induzindo um campo de vetores na esfera . . . . . . . . . . . . . . 23

2 Caracterização dos campos vetoriais $\quad 31$

2.1 Elipse . . . . . . . . . . . . . . . . . . . . . . . . 39

2.2 Elipse Complexa . . . . . . . . . . . . . . . . . . 44

2.3 Hipérbole . . . . . . . . . . . . . . . . . . . . . . . . 46

2.4 Duas retas complexas . . . . . . . . . . . . . . . . . . 58

2.5 Duas retas reais com intersecção . . . . . . . . . . . . . . . . . . . . 61

2.6 Parábola . . . . . . . . . . . . . . . . . . . . . . . . . . . . . . . . . . . 69

2.7 Duas retas paralelas . . . . . . . . . . . . . . . . . . . . . 81

2.8 Duas retas complexas paralelas . . . . . . . . . . . . . . . . 85

2.9 Uma reta dupla . . . . . . . . . . . . . . . . . . . . . . . . . . 88

2.10 Infinito degenerado . . . . . . . . . . . . . . . . . . . 88

2.11 Considerações Finais . . . . . . . . . . . . . . . . . . 97

$\begin{array}{lll}3 & \text { Sobre a técnica utilizada } & 99\end{array}$ 
Referências Bibliográficas

Índice Remissivo 


\section{Lista de Figuras}

1.1 Singularidade do tipo nó-instável. . . . . . . . . . . . . . . . . . . 8

1.2 Singularidade do tipo sela-nó. . . . . . . . . . . . . . . . . 8

1.3 Ponto singular do tipo cúspide. . . . . . . . . . . . . . . . . . . 10

1.4 Ponto singular do tipo sela-nó. . . . . . . . . . . . . . . . . . . 10

1.5 Ponto singular do tipo sela. . . . . . . . . . . . . . . . . . . . . . . 10

1.6 Ponto singular do tipo centro. . . . . . . . . . . . . . . . . . . . 10

1.7 Ponto singular do tipo sela eliptica. . . . . . . . . . . . . . . . . 10

1.8 Ponto singular do tipo foco. . . . . . . . . . . . . . . . . 10

1.9 Retratos de fase dos campos vetoriais homogêneos quadráticos no disco de Poincaré. . . . . . . . . . . . . . . . . . . . . 11

1.10 Exemplo de blow up polar . . . . . . . . . . . . . . . . . . . . . . . 14

1.11 Exemplo de blow up polar . . . . . . . . . . . . . . . . . . . . 15

1.12 Exemplo de blow up direcional . . . . . . . . . . . . . . . . . . . . . . . . . 17

1.13 Relação entre blow up na direção $x$ e blow up polar. . . . . . . . . . . . . . 18

1.14 Relação entre blow up na direção y e blow up polar. . . . . . . . . . . . . . . 19

1.15 Exemplo de blow ups direcionais sucessivos . . . . . . . . . . . . . . . 21

1.16 Exemplo de blow ups direcionais sucessivos . . . . . . . . . . . . . . . 23

1.17 Projeção Central . . . . . . . . . . . . . . . . . . . . . . 24

1.18 Retrato de fase global no disco de Poincaré. . . . . . . . . . . . . . . . . 29

2.1 Retratos de fase globais dos campos quadráticos com integral primeira racional de grau 2 no disco de Poincaré. . . . . . . . . . . . . . . . . . . . . 32

2.2 Pontos singulares infinitos para o caso $(E) \ldots \ldots \ldots$. . . . . . . . 40

2.3 Retratos de fase no disco de Poincaré para o caso $(E)$ com $q>0$. . . . . 42 
2.4 Retratos de fase no disco de Poincaré para o caso $(E)$ com $q<0$. . . . . 42

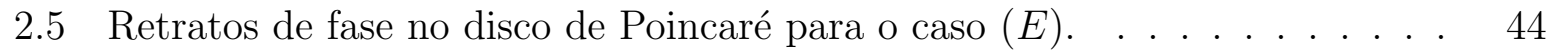

2.6 Blow up na direção $Z_{1}$. . . . . . . . . . . . . . . . . . . . . . . . . . . . . . 50

2.7 Caso $p>0 \ldots \ldots \ldots \ldots \ldots \ldots$

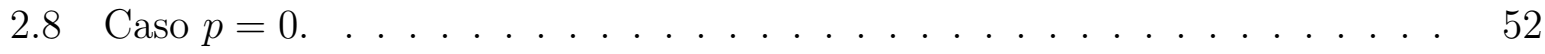

2.9 Pontos singulares infinitos para o caso $(H) \ldots \ldots \ldots \ldots$

2.10 Pontos singulares finitos para o caso $(H)$ com $q>0 \ldots \ldots$. . . . . . 55

2.11 Retrato de fase no disco de Poincaré para o caso $(H) \ldots \ldots$. . . . . . . 57

2.12 Retratos de fase no disco de Poincaré para o caso $(H)$. . . . . . . . . . . 57

2.13 Retratos de fase no disco de Poincaré para o caso $(H) \ldots \ldots$. . . . . . 58

2.14 Blow ups nas direções $Z_{1}$ e $Z_{2}$. . . . . . . . . . . . . . . . . . . . . . . . . 64

2.15 Blow up para o caso $b_{01}=0$ e $b_{00} \neq 0 \ldots \ldots \ldots \ldots$. . . . . . . . 65

2.16 Pontos singulares infinitos para o caso $(L V) \ldots \ldots$. . . . . . . . . . 66

2.17 Pontos singulares finitos para o caso $(L V)$ com $b_{00}>0$ e $b_{01}>0 \ldots \ldots 6$

2.18 Retrato de fase no disco de Poincaré para o caso $(L V)$. . . . . . . . . . 68

2.19 Retrato de fase no disco de Poincaré para o caso $(L V)$. . . . . . . . . . . 68

2.20 Retrato de fase no disco de Poincaré para o caso $(L V)$. . . . . . . . . . 69

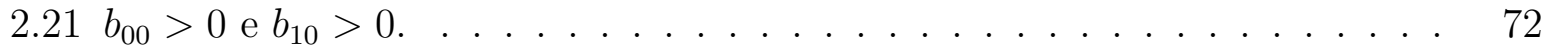

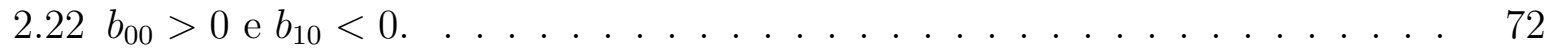

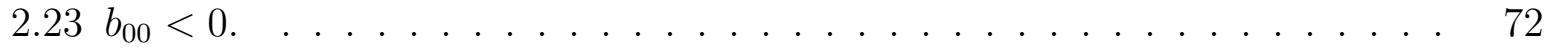

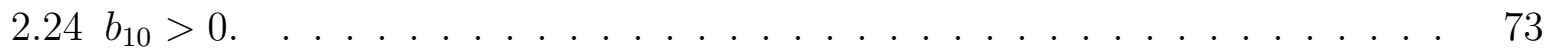

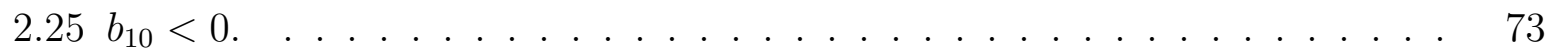

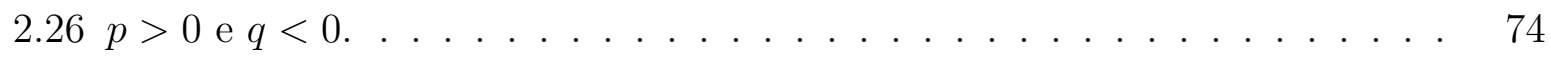

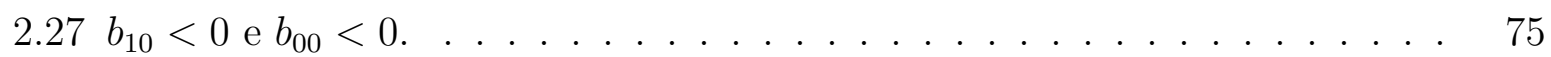

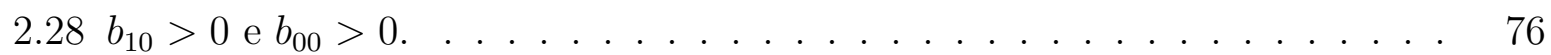

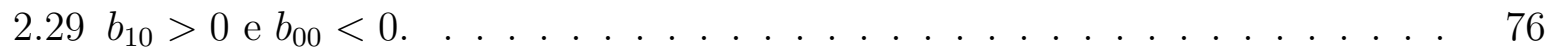

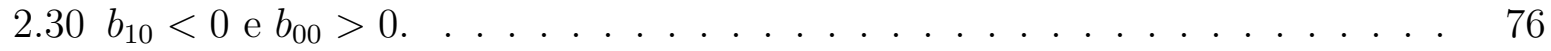

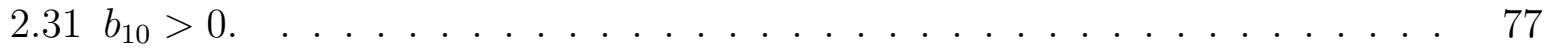

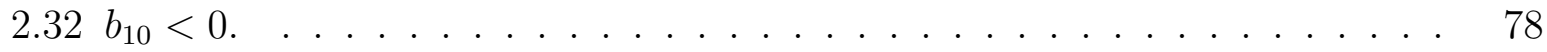

2.33 Pontos singulares infinitos para o caso $(P) \operatorname{com} p>0$ e $q>0$. . . . . 78

2.34 Pontos singulares infinitos para o caso $(P) \operatorname{com} p>0$ e $q<0$. . . . . . 79

2.35 Pontos singulares infinitos para o caso $(P) \operatorname{com} p=0$ e $q>0$. . . . . . 79

2.36 Retratos de fase no disco de Poincaré para o caso $(P)$. . . . . . . . . 81 
2.37 Retratos de fase no disco de Poincaré para o caso $(P)$. . . . . . . . . 81

2.38 Retratos de fase no disco de Poincaré para o caso $(P)$. . . . . . . . . . 81

2.39 Pontos singulares infinitos para o caso $(P L)$ com $b_{01}>0$. . . . . . . . 83

2.40 Pontos singulares finitos para o caso $(P L)$ com $b_{01}>0$. . . . . . . . . 84

2.41 Retratos de fase no disco de Poincaré para o caso $(P L)$ com $b_{01} \neq 0$. . . $\quad 85$

2.42 Retratos de fase no disco de Poincaré para o caso $(C L)$ com $b_{01} \neq 0 \ldots$. . 87

2.43 Retrato de fase no disco de Poincaré para o caso $(D I)$. . . . . . . . . . 90

2.44 Retrato de fase no disco de Poincaré para o caso $(D I) \ldots \ldots$. . . . . . . . 92

2.45 Retrato de fase no disco de Poincaré para o caso $(D I) \ldots \ldots$. . . . . . . . . 92

2.46 Retrato de fase no disco de Poincaré para o caso $(D I)$. . . . . . . . . . 93

2.47 Retrato de fase no disco de Poincaré para o caso $(D I)$. . . . . . . . . . . 94

2.48 Retratos de fase no disco de Poincaré para o caso $(D I)$. . . . . . . . . . . 94

2.49 Retrato de fase no disco de Poincaré para o caso $(D I) \ldots \ldots$. . . . . . . 96 



\section{Introdução}

Facilmente deparamo-nos com equações diferenciais modelando fenômenos físicos, biológicos, dentre outros. Tais equações são estudadas com o objetivo de responder como tais fenômenos evoluem em tempo futuro.

Do estudo quantitativo de uma equação, temos resposta sobre a existência de solução para um dado sistema. Apesar dos esforços de muitos matemáticos na busca de métodos que permitissem-nos resolver equações diferenciais ordinárias (EDO's) particulares, poucas delas têm soluções representadas em termos de funções elementares.

Esta é uma das razões para o aparecimento da teoria qualitativa das EDO's. Essa teoria consiste no estudo do comportamento das soluções de um sistema de EDO's sem o cálculo explícito de sua solução, utilizando apenas certas propriedades dos campos de vetores que o define.

Nessa dissertação estamos interessados nos sistemas diferenciais polinomiais planares da forma

$$
\frac{d x}{d t}=P(x, y) \quad \frac{d y}{d t}=Q(x, y)
$$

onde $P$ e $Q$ são polinômios nas variáveis $x$ e $y$ e o máximo grau de $P$ e $Q$ é $m$.

Um dos principais problemas na teoria qualitativa das equações diferenciais em dimensão dois é apresentar, para uma dada família de sistemas diferenciais, uma classificação topológica dos retratos de fase de todos os sistemas dessa família. Grosseiramente podemos dizer que dois sistemas diferenciais planos são topologicamente equivalentes se existe um homeomorfismo do plano no plano que leva curvas soluções de um sistema em curvas soluções do outro sistema, preservando a orientação em $t$. Portanto, desejamos particionar o espaço dos parâmetros da família de sistemas em subconjuntos, de modo que dois ele- 
mentos da família estejam no mesmo subconjunto se forem topologicamente equivalentes.

Muitos pesquisadores consideraram a classificação topológica de sistemas diferenciais planos em geral, e esses trabalhos geraram o ponto de partida para obtermos todas as classes de equivalência para famílias particulares de sistemas diferenciais planos.

A classificação topológica dos campos de vetores lineares planos é conhecida, porém a classificação topológica dos campos de vetores de grau $n$ no plano, para $n>1$ é um problema em aberto. O caso $n=2$ foi investigado intensivamente, e mais de mil artigos foram publicados sobre esses campos vetoriais, mas a classificação de todos os campos vetoriais quadráticos (de grau 2) ainda é desconhecida. Por este motivo, surge o interesse em estudar os sistemas polinomiais quadráticos planos que satisfaçam propriedades especiais, tais como aqueles que possuem uma integral primeira polinomial ou racional; para mais detalhes veja [5], [7], [13], [17].

Nesta dissertação optamos pelo estudo da referência [5] de Cairó e Llibre com o objetivo de aprender técnicas utilizadas na classificação dos retratos de fase globais dos sistemas diferenciais planos. Na presente referência os autores obtêm a classificação de todos os retratos de fase globais dos campos de vetores polinomiais quadráticos que possuem uma integral primeira racional de grau 2.

Essa dissertação está organizada da seguinte maneira:

No CAPítulo 1, introduzimos as notações, apresentamos definições básicas tais como integral primeira racional, campo de vetores polinomial quadrático, curvas algébricas invariantes e outros. Apresentamos também a classificação de pontos singulares, descrevemos a compactificação de Poincaré e a técnica conhecida como Blow up. O objetivo desse capítulo é fornecer as ferramentas necessárias para a leitura dos capítulos seguintes.

No Capítulo 2, apresentamos, de maneira detalhada, a técnica utilizada para a obtenção da classificação de todos os retratos de fase globais dos campos vetoriais quadráticos que possuem uma integral primeira racional de grau 2.

O CApítulo 3, é devotado a comentar alguns trabalhos que classificam o retrato de fase global de outras classes de sistemas polinomiais planos. Vale observar que em todos os trabalhos comentados nesse capítulo a técnica para obtenção dos retratos de fase global é a mesma apresentada no capítulo anterior. A diferença se encontra na maneira como se obtem as formas normais dos sistemas desejados. 


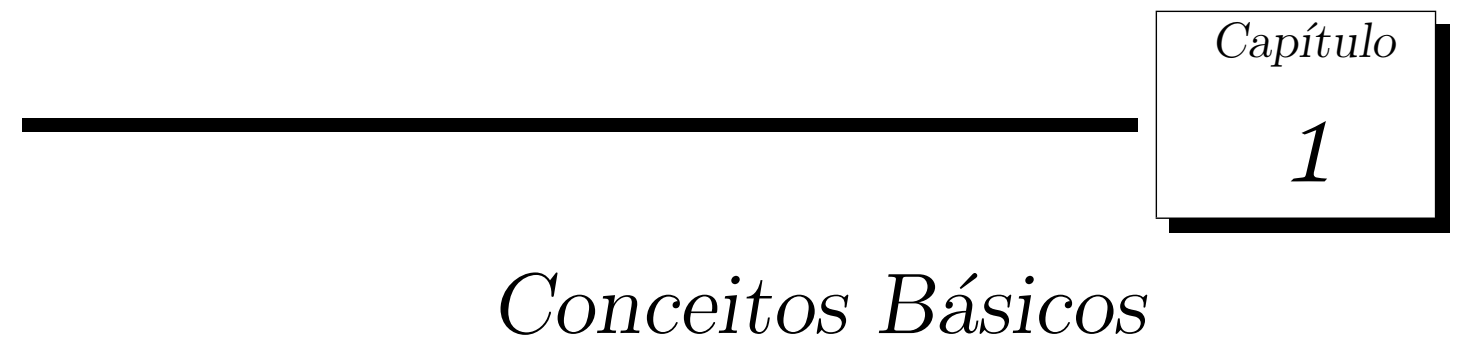

O objetivo deste capítulo é apresentar os conceitos e resultados indispensáveis para o entendimento dos assuntos abordados neste trabalho. Com intuito de não fugir de nosso objetivo omitimos as provas de alguns desses resultados e as substituímos por suas respectivas referências. As principais referências são [2], [11] e [20].

\subsection{Integral primeira e curvas invariantes}

Definição 1.1.1. Um campo de vetores em $U \subset \mathbb{R}^{2}$, aberto, é uma aplicação $X: U \rightarrow \mathbb{R}^{2}$.

Definição 1.1.2. Uma trajetória ou curva integral de um campo de vetores $X: U \rightarrow \mathbb{R}^{2}$, é uma curva diferenciável $\gamma: I \rightarrow U$ definida em um intervalo real não degenerado tal que:

$$
\gamma^{\prime}(t)=X(\gamma(t)) \forall t \in I
$$

Definição 1.1.3. Sejam $X: U \rightarrow \mathbb{R}^{2}$ um campo, $x_{0} \in U$ e $t_{0} \in \mathbb{R}$ dados. Dizemos que uma trajetória $\varphi: I \rightarrow U$ de $X$ é uma trajetória maximal de $X$ por $x_{0}$ em $t_{0}$ se $\varphi$ é uma trajetória de $X$ por $x_{0}$ em $t_{0}$ que estende qualquer outra, ou seja, se $\psi: J \rightarrow U$ é uma trajetória de $X$ por $x_{0}$ em $t_{0}$ então necessariamente $J \subseteq I$ e $\psi=\varphi \mid J$. Denotaremos por $I\left(x_{0}, t_{0}\right)$ o intervalo maximal de $X$ por $x_{0}$ em $t_{0}$.

Definição 1.1.4. A trajetória maximal de $X$ por $x$ é a trajetória maximal de $X$ por $x$ em 0 , para cada $x \in U$ e denotaremos por $\varphi_{x}: I(x) \rightarrow U$ onde $I(x)=I(x, 0)$.

Definição 1.1.5. Seja $\Omega=\{(t, x) \in \mathbb{R} \times U ; t \in I(x)\}$. A aplicação $\varphi: \Omega \rightarrow U$ definida por $\varphi(t, x)=\varphi_{x}(t)$ é denominada fluxo de $X$.

Definição 1.1.6. Um conjunto $M$ é invariante se para todo $x \in M, \varphi(x, t) \in M$ para todo $t$. 
Seja $\mathbb{R}[x, y]$ o anel dos polinômios nas variáveis $x$ e $y$ com coeficientes em $\mathbb{R}$. Considere o sistema de equações diferenciais polinomial em $\mathbb{R}^{2}$ definido por:

$$
\left\{\begin{array}{l}
\dot{x}=P(x, y) \\
\dot{y}=Q(x, y)
\end{array}\right.
$$

onde $P, Q \in \mathbb{R}[x, y]$ têm grau $m$ e $n$, respectivamente. Dizemos que $r=\max \{m, n\}$ é o grau do sistema (1.1).

Ao invés de falarmos do sistema (1.1), frequentemente falamos do campo vetorial polinomial associado a ele

$$
X=P(x, y) \frac{\partial}{\partial x}+Q(x, y) \frac{\partial}{\partial y} .
$$

Outra maneira de expressar o campo vetorial é escrevê-lo como $X=(P, Q)$. Neste trabalho, não faremos distinção entre o sistema (1.1) e o campo vetorial (1.2).

Um campo vetorial polinomial quadrático é um campo de vetores polinomial para o qual $r=2$.

Definição 1.1.7. Dada $f \in \mathbb{R}[x, y]$, dizemos que a curva $f(x, y)=0$ é uma curva algébrica invariante do sistema (1.1), se existe $k \in \mathbb{R}[x, y]$ tal que:

$$
P \frac{\partial f}{\partial x}+Q \frac{\partial f}{\partial y}=k f
$$

Exemplo 1.1.8. Considere o sistema diferencial

$$
\left\{\begin{array}{l}
\dot{x}=-x^{2} \\
\dot{y}=y^{2}
\end{array}\right.
$$

Então dadas $f_{1}(x, y)=x+y, f_{2}(x, y)=x$ e $f_{3}(x, y)=y$ segue que

$$
\begin{aligned}
& P \frac{\partial f_{1}}{\partial x}+Q \frac{\partial f_{1}}{\partial y}=(-x+y)(x+y)=k_{1} f_{1} \\
& P \frac{\partial f_{2}}{\partial x}+Q \frac{\partial f_{2}}{\partial y}=-x^{2}=k_{2} f_{2} \\
& P \frac{\partial f_{2}}{\partial x}+Q \frac{\partial f_{2}}{\partial y}=y^{2}=k_{3} f_{3}
\end{aligned}
$$


onde $k_{1}(x, y)=-x+y$ e $k_{2}(x, y)=-x$ e $k_{3}(x, y)=y$. Portanto, $f_{1}(x, y)=0, f_{2}(x, y)=0$ e $f_{3}(x, y)=0$ são retas invariantes do sistema (1.3).

Definição 1.1.9. Seja $U$ um subconjunto aberto e denso de $\mathbb{R}^{2}$, dizemos que uma função não constante $H: U \rightarrow \mathbb{R}$ é uma integral primeira do sistema (1.1) sobre $U$, se $H(x(t), y(t))$ é constante ao longo das soluções $(x(t), y(t))$ desse sistema, contidas em $U$, isto é, toda trajetória de $X$, interseccionada com $U$, está contida em uma curva de nível de $H$.

Observação 1.1.10. Sejam $c, k$ constantes quaisquer, com $c \neq 0$, e suponha que $H(x(t), y(t))=$ $c$, onde $(x(t), y(t))$ são as curvas soluções do sistema (1.1). Então:

i) $k H, k+H, \frac{1}{H}$ são integrais primeiras de (1.1).

Basta notar que

$$
\begin{aligned}
& (k H)(x(t), y(t))=k(H(x(t), y(t))=k c \\
& (k+H)(x(t), y(t))=k+H(x(t), y(t))=k+c \mathrm{e} \\
& \frac{1}{H}(x(t), y(t))=\frac{1}{H(x(t), y(t))}=\frac{1}{c} .
\end{aligned}
$$

ii) $H$ é integral primeira do sistema (1.1) se, e somente se, existe $R$ tal que $P=$ $-\frac{\partial H}{\partial y} R(x, y)$ e $Q=\frac{\partial H}{\partial x} R(x, y)$, onde o domínio de $R$ é igual ao domínio de $H$.

De fato, se $H$ é integral primeira de (1.1) então:

$$
\frac{\partial H}{\partial x}(x, y) \dot{x}+\frac{\partial H}{\partial y}(x, y) \dot{y}=0 \Leftrightarrow \frac{\partial H}{\partial x}(x, y) P(x, y)+\frac{\partial H}{\partial y}(x, y) Q(x, y)=0
$$

e, isto equivale a dizer que para cada $\left(x_{0}, y_{0}\right)$ no domínio de $H$ (aberto e denso em $\left.\mathbb{R}^{2}\right)$ o vetor $\left(P\left(x_{0}, y_{0}\right), Q\left(x_{0}, y_{0}\right)\right)$ é ortogonal ao vetor $\left(\frac{\partial H}{\partial x}\left(x_{0}, y_{0}\right), \frac{\partial H}{\partial y}\left(x_{0}, y_{0}\right)\right)$.

O vetor $\left(-\frac{\partial H}{\partial y}\left(x_{0}, y_{0}\right), \frac{\partial H}{\partial x}\left(x_{0}, y_{0}\right)\right)$ é ortogonal ao vetor $\left(\frac{\partial H}{\partial x}\left(x_{0}, y_{0}\right), \frac{\partial H}{\partial y}\left(x_{0}, y_{0}\right)\right)$. Como trabalhamos em $\mathbb{R}^{2}$, concluímos que qualquer outro vetor nessa condição será um múltiplo de $\left(-\frac{\partial H}{\partial y}\left(x_{0}, y_{0}\right), \frac{\partial H}{\partial x}\left(x_{0}, y_{0}\right)\right)$, a cada ponto $\left(x_{0}, y_{0}\right)$.

Portanto $P=-\frac{\partial H}{\partial y}(x, y) R(x, y)$ e $Q=\frac{\partial H}{\partial x}(x, y) R(x, y)$, a cada $(x, y)$ no domínio de $H$. 
iii) Uma curva $\alpha(t)=(x(t), y(t))$, tal que $H(x(t), y(t))=0$, é uma curva algébrica invariante do sistema (1.1).

É possível encontrar a integral primeira de um sistema diferencial polinomial usando suas curvas algébricas invariantes. O problema já foi considerado por Darboux [9] em 1978 e outros pesquisadores apresentam versões do Teorema de Darboux. Por exemplo, Jouanolou [16], em 1979, mostrou que, se o número de curvas algébricas invariantes de um sistema diferencial polinomial de grau $m$ é pelo menos $\left[\frac{m(m+1)}{2}\right]+2$, então, o sistema admite uma integral primeira racional.

Para o sistema (1.1) definido no plano, a existência de uma integral primeira determina completamente seu retrato de fase, isto é, a decomposição do plano na união das órbitas de (1.1).

O trabalho de Cairó e Llibre, [5], tem por objetivo classificar os retratos de fase global no disco de Poincaré dos campos vetoriais quadráticos com integral primeira $H$ racional de grau 2. Isto é, $H$ é da forma

$$
H=\frac{a_{00}+a_{10} x+a_{01} y+a_{20} x^{2}+a_{11} x y+a_{02} y^{2}}{b_{00}+b_{10} x+b_{01} y+b_{20} x^{2}+b_{11} x y+b_{02} y^{2}}
$$

onde $a_{20}^{2}+a_{11}^{2}+a_{02}^{2}+b_{20}^{2}+b_{11}^{2}+b_{02}^{2} \neq 0$ e o numerador e denominador são polinômios de grau maior que 1 , isto é, $H$ e $\frac{1}{H}$ não são polinômios.

Notamos que os campos vetoriais quadráticos com integral primeira racional de grau 2 têm todas as suas órbitas contidas em cônicas. Portanto, suas órbitas são curvas muito simples, mas isto não impede que seus retratos de fase possam apresentar uma rica dinâmica como descrevemos aqui.

Para dissertar sobre o tema proposto apresentamos abaixo a classificação local dos pontos singulares; os conceitos de blow up e compactificação de Poincaré bem como exemplos.

\subsection{Pontos singulares}

Um ponto $p \in \mathbb{R}^{2}$ é dito ponto singular do campo vetorial $X=(P, Q)$ se $X(p)=0$.

Seja $J X(p)$ a matriz Jacobiana do campo $X$, isto é,

$$
J X(p)=\left[\begin{array}{ll}
P_{x}(p) & P_{y}(p) \\
Q_{x}(p) & Q_{y}(p)
\end{array}\right]
$$


onde $P_{x}, Q_{x}$ e $P_{y}, Q_{y}$ denotam as derivadas parciais de $P$ e $Q$ com respeito às variáveis $x$ e $y$, respectivamente.

Defina $\Delta=\operatorname{det} J X(p)=P_{x}(p) Q_{y}(p)-P_{y}(p) Q_{x}(p)$ e $T=\operatorname{Tra} J X(p)=P_{x}(p)+Q_{y}(p)$.

Um ponto singular $p$ é dito não degenerado se $\Delta \neq 0$. Então, $p$ é um ponto singular isolado. Nesse caso, $p$ é uma sela se $\Delta<0$, um nó se $T^{2} \geq 4 \Delta>0$ (estável, se $T<0$, e instável, se $T>0$ ), um foco se $4 \Delta>T^{2}>0$ (estável se $T<0$, instável, se $T>0$ ), e um foco fraco ou um centro, se $T=0<\Delta$; para mais detalhes veja [2], p.183.

O ponto singular $p$ é chamado hiperbólico se os autovalores de $J X(p)$ têm parte real diferente de zero. Então, os pontos singulares hiperbólicos são os não degenerados, exceto o foco fraco e o centro.

Um ponto singular $p$ degenerado (i.é., $\Delta=0$ ) com $T \neq 0$ é chamado semi-hiperbólico, e $p$ é isolado no conjunto de todos os pontos singulares.

Se $X=(P, Q)$ é um campo de vetores polinomial de grau $n$ e $(0,0)$ é ponto singular de $X$, do tipo semi-hiperbólico, então existe uma mudança de coordenadas reduzindo $X$ ao campo $\bar{X}=(F(x, y), y+G(x, y))$, onde $F(x, y), G(x, y)$ são polinômios e suas expansões em séries envolvem termos de ordem maior ou igual a 2 .

Para caracterizar o retrato de fase dos pontos singulares semi-hiperbólicos numa vizinhança da origem, temos a seguinte proposição (para uma prova, veja Teorema 65 de [2]):

Proposição 1.2.1. Seja $(0,0)$ um ponto singular isolado do campo vetorial $(F(x, y), y+$ $G(x, y))$, onde $F$ e $G$ são funções analíticas numa vizinhança da origem, com suas expansões em séries de potências em torno de $(0,0)$ começando com termos no mínimo quadráticos nas variáveis $x$ e $y$. Seja $y=g(x)$ uma solução da equação $y+G(x, y)=0$ numa vizinhança de $(0,0)$. Assuma que o desenvolvimento da função $f(x)=F(x, g(x))$ é da forma $f(x)=\mu x^{m}+O\left(x^{m}\right)$, onde $m \geq 2, \mu \neq 0$ e $O\left(x^{m}\right)$ denota os termos de ordem maior que $x^{m}$. Quando $m$ é ímpar, então $(0,0)$ é um nó instável se $\mu>0$ (veja Figura 1.1) e uma sela se $\mu<0$ (veja Figura 1.5). No caso da sela, as separatrizes estáveis são tangentes ao eixo x. Se $m$ é par, então $(0,0)$ é uma sela-nó, i.e., o ponto singular é formado pela união de dois setores hiperbólicos com um setor parabólico. A separatriz estável é tangente ao eixo $x$ positivo (respectivamente negativo) em $(0,0)$, se $\mu<0$ (respectivamente $\mu>0)$. As duas separatrizes instáveis são tangentes ao eixo y em $(0,0)$, veja Figura 1.2. 


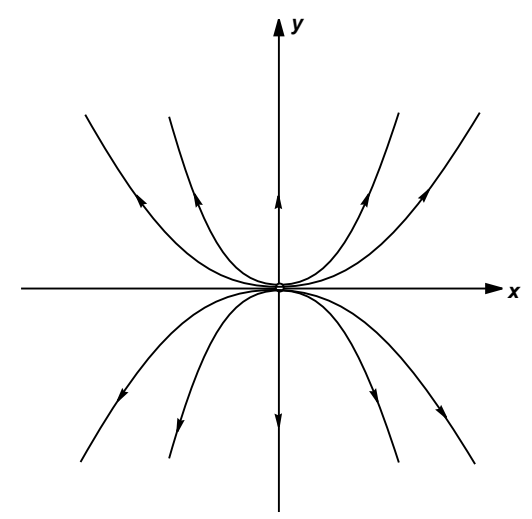

Figura 1.1: Singularidade do tipo nó-instável.

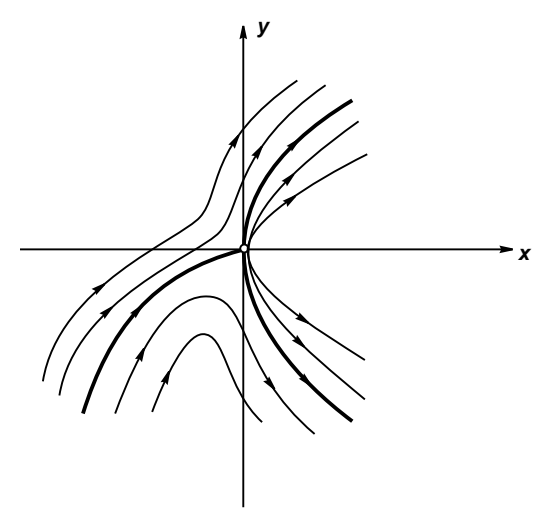

Figura 1.2: Singularidade do tipo sela-nó.

Os pontos singulares que são não-degenerados ou semi-hiperbólicos são chamados elementares.

Quando $\Delta=T=0$, mas $J X(p) \neq 0$ e $p$ é isolado no conjunto de todos os pontos singulares, dizemos que $p$ é nilpotente.

Seja $X=(P, Q)$ campo de vetores polinomial de grau $n$. Suponha que $(0,0)$ é ponto singular de $X$, do tipo nilpotente. Então sob tais condições existe uma mudança de coordenadas reduzindo $X$ ao campo $\bar{X}=(y+F(x, y), G(x, y))$, onde $F(x, y), G(x, y)$ são polinômios e suas expansões em séries envolvem termos de ordem maior ou igual a 2 .

A seguir, resumimos a classificação dos pontos singulares nilpotentes. Para uma prova, veja Teoremas 66 e 67 e a versão simplificada da Seção 22.3 de [2].

Proposição 1.2.2. Seja $(0,0)$ um ponto singular isolado do campo vetorial $(y+F(x, y), G(x, y))$, onde $F$ e $G$ são funções analíticas numa vizinhança da origem, com suas expansões em séries, começando com termos no mínimo quadráticos nas variáveis $x$ e $y$. Seja $y=f(x)$ uma solução da equação $y+F(x, y)=0$ numa vizinhança de $(0,0)$. Assuma que o desenvolvimento da função $G(x, f(x))$ é da forma $K x^{\kappa}+O\left(x^{\kappa}\right)$ e $\Phi(x) \equiv\left(\frac{\partial F}{\partial x}+\frac{\partial G}{\partial y}\right)(x, f(x))=L x^{\lambda}+O\left(x^{\lambda}\right)$, onde $\kappa \geq 2, K \neq 0$ e $O\left(x^{\kappa}\right), O\left(x^{\lambda}\right) d e-$ notam respectivamente os termos de ordem maior que $x^{\kappa}$ e $x^{\lambda}$. Então, valem as seguintes afirmações: 
(1) Se к é par

(1.a) $\kappa>2 \lambda+1$, então a origem é uma sela-nó. Além disso a sela-nó tem uma separatriz tangente ao semi-eixo $x<0$, e as outras duas separatrizes tangentes ao semi-eixo $x>0$ (veja Figura 1.4).

(1.b) $\kappa<2 \lambda+1$ ou $\Phi \equiv 0$, então a origem é uma cúspide, i.e., um ponto singular formado pela união de dois setores hiperbólicos. Além disso, a cúspide tem duas separatrizes tangentes ao semi-eixo $x$ positivo (veja Figura 1.3).

(2) Se $\kappa$ é impar e $K>0$, então a origem é uma sela. Além disso, a sela tem duas separatrizes tangentes ao semi-eixo $x<0$, e as outras duas tangentes ao semi-eixo $x>0$ (veja Figura 1.5).

(3) Se $\kappa$ é impar, $K<0$ e

(3.a) $\lambda$ par, $\kappa=2 \lambda+1$ e $L^{2}+4 K(\lambda+1) \geq 0$, ou $\lambda$ par e $\kappa>2 \lambda+1$, então a origem é um nó estável (instável) se $L<0$ ( $L>0)$, com as órbitas tangentes ao eixo $x$ em $(0,0)$ (veja Figura 1.1).

(3.b) $\lambda$ impar, $\kappa=2 \lambda+1$ e $L^{2}+4 K(\lambda+1) \geq 0$, ou $\lambda$ impar e $\kappa>2 \lambda+1$, então a origem é uma sela elíptica, i.e., um ponto singular formado pela união de um setor hiperbólico e um setor elíptico. Além disso, uma separatriz da sela-elíptica é tangente ao semi-eixo $x<0$ e a outra ao semi-eixo $x>0$ (veja Figura 1.7).

(3.c) $\kappa=2 \lambda+1$ e $L^{2}+4 K(\lambda+1)<0$, ou $\kappa<2 \lambda+1$, então a origem é um foco (veja Figura 1.8) ou um centro, e se $\Phi \equiv 0$ então a origem é um centro (veja Figura 1.6).

Finalmente, se $J X(p)=0$ e $p$ é isolado no conjunto de todos os pontos singulares, dizemos que $p$ é linearmente nulo. O estudo do seu retrato de fase local precisa de um tratamento especial, blow ups direcionais, veja Seção 1.3 para mais detalhes.

Mas, se o campo vetorial quadrático tem um ponto singular linearmente nulo finito, então, ele é equivalente ao campo vetorial quadrático homogêneo depois de uma translação do ponto singular linearmente nulo para a origem. Os retratos de fase globais dos campos vetoriais homogêneos quadráticos foram estudados por Ye Yan Quian em [22]. A Figura 1.9 apresenta a classificação de tais campos no disco de Poincaré. Para mais detalhes sobre o disco de Poincaré veja Seção 1.4. 


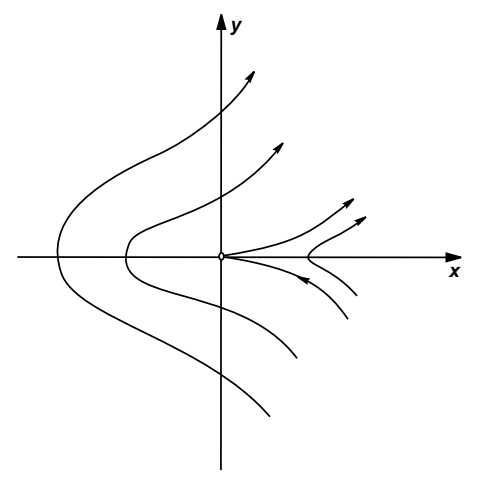

Figura 1.3: Ponto singular do tipo cúspide.

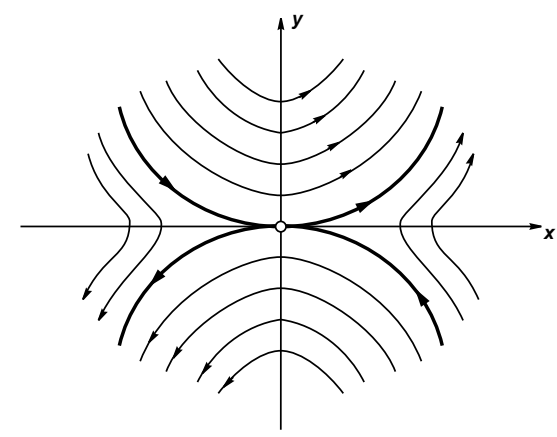

Figura 1.5: Ponto singular do tipo sela.

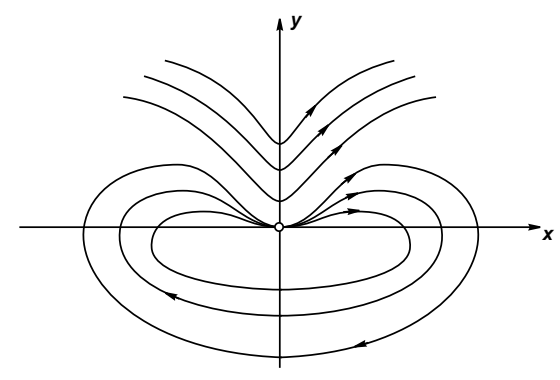

Figura 1.7: Ponto singular do tipo sela eliptica.

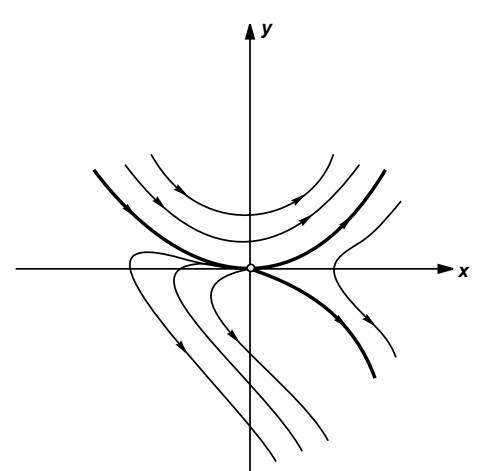

Figura 1.4: Ponto singular do tipo sela-nó.

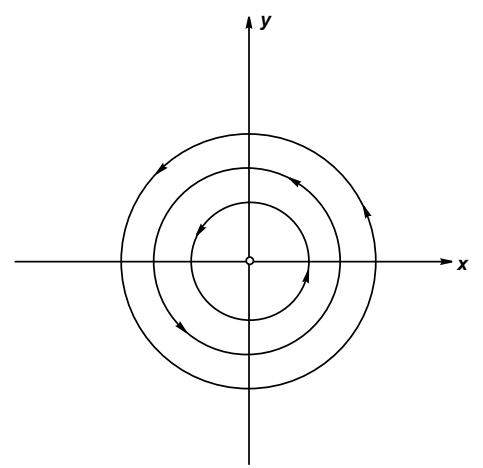

Figura 1.6: Ponto singular do tipo centro.

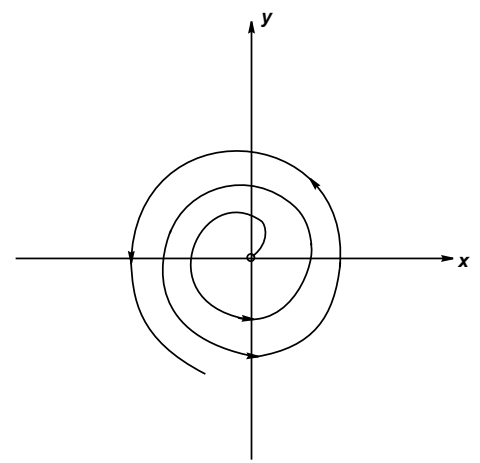

Figura 1.8: Ponto singular do tipo foco. 

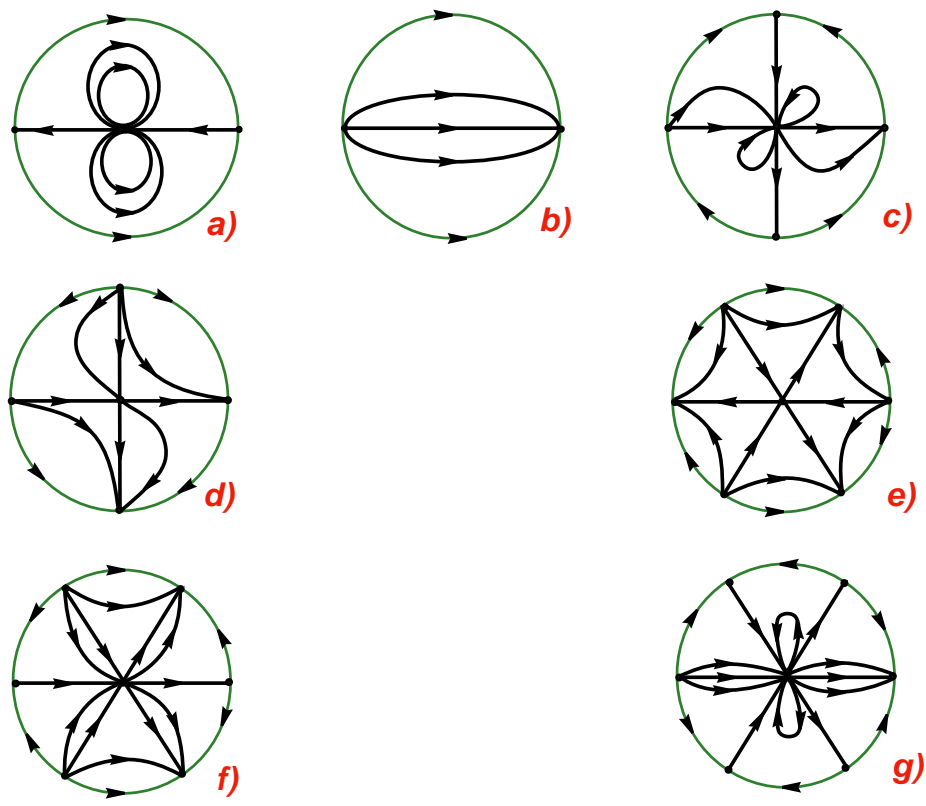

Figura 1.9: Retratos de fase dos campos vetoriais homogêneos quadráticos no disco de Poincaré.

\subsection{Blow up}

Podemos dizer que blow up é a técnica na qual uma mudança de coordenadas expande ou "explode" uma singularidade, em uma curva na qual temos, genericamente, finitas singularidades. Após a mudança de coordenadas, estudamos as singularidades isoladamente do novo campo $\bar{X}$ ao longo dessa curva e se uma singularidade é não hiperbólica, podemos aplicar um novo blow up na mesma, e assim, sucessivamente. Sob condições genéricas, após uma sequência finita de blow ups serão encontradas apenas singularidades elementares. Esse resultado é conhecido como Teorema da Decomposição. Finalmente, pelo processo inverso (blowing down), obtemos o retrato de fase local de $X$ numa vizinhança do ponto singular degenerado. Sobre blow up e Teorema da Decomposição veja detalhes em [11].

\subsubsection{Blow up polar}

Seja $X=(P(x, y), Q(x, y))$ um campo de vetores polinomial em $\mathbb{R}^{2}$.

Suponha que $X$ tem singularidade isolada em $(0,0)$.

Considere a aplicação: 


$$
\begin{array}{rlc}
\phi: \mathbb{S}^{1} \times \mathbb{R} & \rightarrow & \mathbb{R}^{2} \\
(r, \theta) & \mapsto(r \cos \theta, r \operatorname{sen} \theta)
\end{array}
$$

Podemos definir um campo de vetores polinomial $\bar{X}$ sobre o cilindro $\mathbb{S}^{1} \times \mathbb{R}$ tal que $\phi_{*}(\bar{X})=X$, no sentido que $D \phi_{v}(\bar{X}(v))=X(\phi(v))$. Este campo é, na verdade, o campo $X$ escrito em coordenadas polares. A aplicação $\phi$ é um difeomorfismo, e portanto, uma autêntica mudança de coordenada em $\mathbb{S}^{1} \times(0, \infty)$, mas não em $\{r=0\} ; \phi$ leva $\{r=0\}$ em $(0,0)$, de maneira que a aplicação inversa $\phi^{-1}$ "explode" a origem em um círculo.

Para estudar o retrato de fase de $X$ numa vizinhança $V$ da origem, é suficiente estudar o retrato fase de $\bar{X}$ na vizinhança $\phi^{-1}(V)$ do círculo $\mathbb{S}^{1} \times\{0\}$.

A seguir encontraremos a expressão do campo $X$ em coordenadas polares.

Se $x=r \cos \theta$ e $y=r \operatorname{sen} \theta$, temos

$$
\left\{\begin{array} { l } 
{ x ^ { 2 } + y ^ { 2 } = r ^ { 2 } } \\
{ \operatorname { t a n } \theta = \frac { y } { x } }
\end{array} \text { e } \left\{\begin{array}{l}
x \dot{x}+y \dot{y}=r \dot{r} \\
\dot{\theta}=\frac{\dot{y} x-\dot{x} y}{r^{2}}
\end{array}\right.\right.
$$

ou seja,

$$
\left\{\begin{array}{l}
P(r \cos \theta, r \operatorname{sen} \theta) r \cos \theta+Q(r \cos \theta, r \operatorname{sen} \theta) r \operatorname{sen} \theta=r \dot{r} \\
Q(r \cos \theta, r \operatorname{sen} \theta) r \cos \theta-P(r \cos \theta, r \operatorname{sen} \theta) r \operatorname{sen} \theta=r^{2} \dot{\theta}
\end{array}\right.
$$

Dividindo o sistema acima por $r$ temos:

$$
\left\{\begin{array}{l}
\dot{r}=P \cos \theta+Q \operatorname{sen} \theta \\
\dot{\theta}=\frac{Q \cos \theta-P \operatorname{sen} \theta}{r}
\end{array}\right.
$$

Chame o campo $\tilde{X}(r, \theta)=\left(X_{r}(r, \theta), \frac{1}{r} X_{\theta}(r, \theta)\right)$, onde $X_{r}=P \cos \theta+Q \operatorname{sen} \theta$ e $X_{\theta}=$ $Q \cos \theta-P \operatorname{sen} \theta$.

Agora, escolha $\kappa$ o maior inteiro positivo tal que $r^{\kappa} \mid P(r \cos \theta, r \operatorname{sen} \theta)$ e $r^{\kappa} \mid Q(r \cos \theta, r \operatorname{sen} \theta)$, e defina $\bar{X}=\frac{\tilde{X}}{r^{\kappa-1}}$.

Com isso, $\bar{X}(r, \theta)=\left(\bar{X}_{r}(r, \theta), \bar{X}_{\theta}(r, \theta)\right)=\left(r \frac{X_{r}}{r^{\kappa}}, \frac{X_{\theta}}{r^{\kappa}}\right)$.

Observe que a singularidade $(0,0)$ foi levada para a curva $\mathbb{S}^{1} \times\{0\}$. Além disso, ao longo de $\mathbb{S}^{1} \times\{0\}$, as singularidades são determinadas pelas soluções de $\bar{X}_{\theta}(0, \theta)=0$.

Alguns exemplos: 
Exemplo 1.3.1. Consideremos o campo $X=\left(x^{2}-2 x y, y^{2}-2 x y\right)$.

Fazendo a mudança de coordenadas $x=r \cos \theta$ e $y=r \operatorname{sen} \theta$ obtemos:

$$
\begin{aligned}
& P(r \cos \theta, r \operatorname{sen} \theta)=r^{2}\left(\cos ^{2} \theta-2 \cos \theta \operatorname{sen} \theta\right) \\
& Q(r \cos \theta, r \operatorname{sen} \theta)=r^{2}\left(\operatorname{sen}^{2} \theta-2 \cos \theta \operatorname{sen} \theta\right)
\end{aligned}
$$

então,

$\tilde{X}(r, \theta)=\left(r^{2}\left(\cos ^{3} \theta+\operatorname{sen}^{3} \theta-2 \cos ^{2} \theta \operatorname{sen} \theta+2 \operatorname{sen}^{2} \theta \cos \theta\right), 3 r \cos \theta \operatorname{sen} \theta(\operatorname{sen} \theta-\cos \theta)\right)$

Observe que $\kappa=2$ é o maior inteiro tal que $r^{\kappa} \mid P(r \cos \theta, r \operatorname{sen} \theta)$ e $r^{\kappa} \mid Q(r \cos \theta, r \operatorname{sen} \theta)$. Portanto, $\bar{X}(r, \theta)=\frac{\tilde{X}(r, \theta)}{r}$, isto é,

$\bar{X}(r, \theta)=\left(r\left(\cos ^{3} \theta+\operatorname{sen}^{3} \theta-2 \cos ^{2} \theta \operatorname{sen} \theta+2 \operatorname{sen}^{2} \theta \cos \theta\right), 3 \cos \theta \operatorname{sen} \theta(\operatorname{sen} \theta-\cos \theta)\right)$.

$\operatorname{Em} r=0, \operatorname{temos} \theta=0, \frac{\pi}{2}, \frac{3 \pi}{2}, \pi, \frac{\pi}{4}, \frac{5 \pi}{4}$, as soluções de $\bar{X}_{\theta}(0, \theta)=0$. Agora, estudamos o campo localmente em cada ponto singular.

$$
J \bar{X}(r, \theta)=\left[\begin{array}{cc}
\frac{\partial \bar{X}_{r}}{\partial r}(r, \theta) & \frac{\partial \bar{X}_{r}}{\partial \theta}(r, \theta) \\
\frac{\partial \bar{X}_{\theta}}{\partial r}(r, \theta) & \frac{\partial \bar{X}_{\theta}}{\partial \theta}(r, \theta)
\end{array}\right]
$$

Como $\frac{\partial \bar{X}_{\theta}}{\partial r}(r, \theta)=0, \operatorname{det} J \bar{X}(r, \theta)=\frac{\partial \bar{X}_{r}}{\partial r}(r, \theta) \frac{\partial \bar{X}_{\theta}}{\partial \theta}(r, \theta)=\left(\cos ^{3} \theta+\operatorname{sen}^{3} \theta-2 \cos ^{2} \theta\right.$ $\left.\operatorname{sen} \theta+2 \operatorname{sen}^{2} \theta \cos \theta\right)\left(6 \operatorname{sen} \theta \cos ^{2} \theta-3 \operatorname{sen}^{3} \theta+6 \cos \theta \operatorname{sen}^{2} \theta-3 \cos ^{3} \theta\right)$.

Então: $\operatorname{det} J \bar{X}(0,0)=\operatorname{det} J \bar{X}(0, \pi)=-3$ e, portanto, $(0,0)$ é singularidades do tipo sela. Da mesma forma, verificamos que para $\theta=\frac{\pi}{2}, \frac{3 \pi}{2}, \frac{\pi}{4}, \frac{5 \pi}{4}$, temos singularidades do tipo sela. Reunindo tais informações e aplicando um blowing down, concluímos que o retrato de fase de $X$ é dado pela Figura 1.10. 


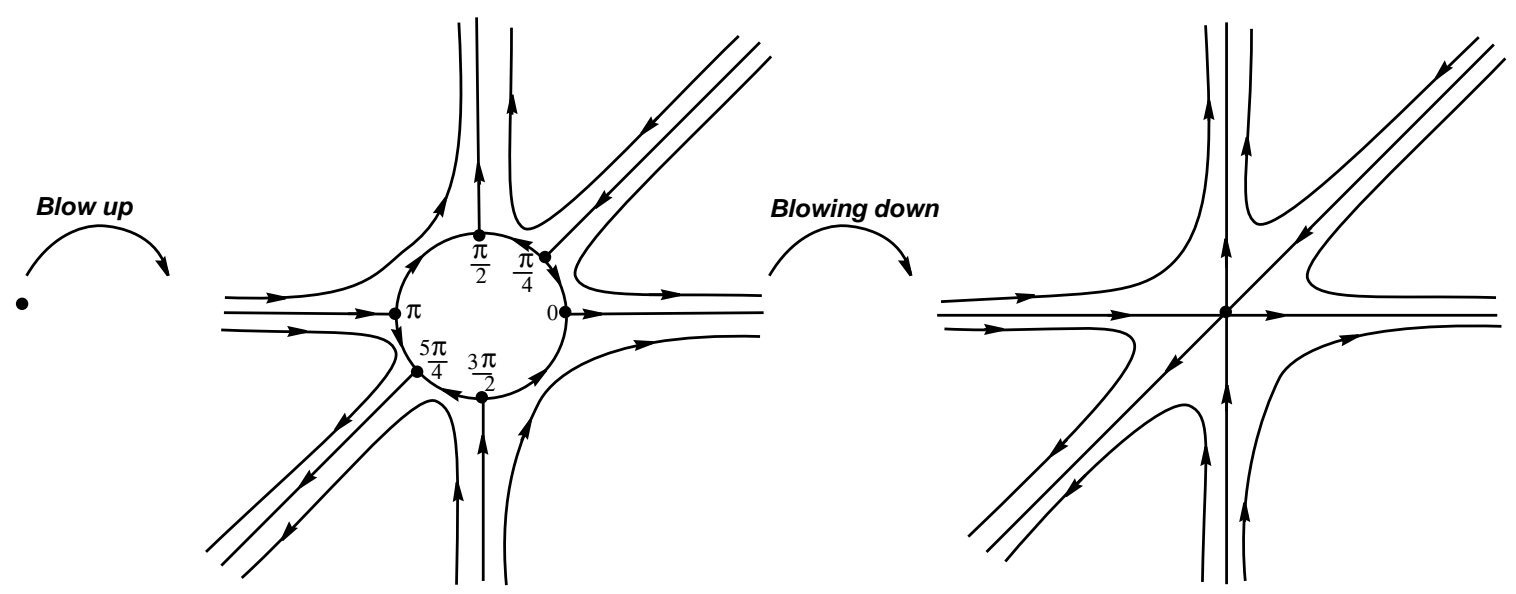

Figura 1.10: Exemplo de blow up polar

Exemplo 1.3.2. Consideremos o campo $X=\left(x^{2}-y^{2}, 2 x y\right)$.

Fazendo a mudança de coordenadas $x=r \cos \theta$ e $y=r \operatorname{sen} \theta$ obtemos:

$$
\begin{aligned}
& P(r \cos \theta, r \operatorname{sen} \theta)=r^{2}\left(\cos ^{2} \theta-\operatorname{sen}^{2} \theta\right) \\
& Q(r \cos \theta, r \operatorname{sen} \theta)=2 r^{2} \cos \theta \operatorname{sen} \theta
\end{aligned}
$$

Observe que $\kappa=2$ é o maior inteiro, tal que $r^{\kappa} \mid P(r \cos \theta, r \operatorname{sen} \theta)$ e $r^{\kappa} \mid Q(r \cos \theta, r \operatorname{sen} \theta)$.

Então, $\tilde{X}=\left(r^{2}\left(\cos ^{3} \theta+\operatorname{sen}^{2} \theta \cos \theta\right), r\left(\cos ^{2} \theta \operatorname{sen} \theta+\operatorname{sen}^{3} \theta\right)\right)$.

Logo, $\bar{X}=\frac{\tilde{X}}{r}=\left(r\left(\cos ^{3} \theta+\operatorname{sen}^{2} \theta \cos \theta\right), \cos ^{2} \theta \operatorname{sen} \theta+\operatorname{sen}^{3} \theta\right)$.

Em $r=0$ temos $\theta=0, \pi$, as soluções de $\bar{X}_{\theta}=0$. Para análise local de $\bar{X}(0, \theta)$ em cada ponto singular temos

$$
J \bar{X}(r, \theta)=\left[\begin{array}{cc}
\cos ^{3} \theta+\operatorname{sen}^{2} \theta \cos \theta & -\cos ^{2} \theta \operatorname{sen} \theta-\operatorname{sen}^{3} \theta \\
0 & \operatorname{sen}^{2} \theta \cos \theta+\cos ^{3} \theta
\end{array}\right]
$$

Como det $J \bar{X}(0,0)=1$ e Tra $J \bar{X}(0,0)=2$, a singularidade $(0,0)$ é do tipo nó repulsor. $\mathrm{E} \operatorname{det} J \bar{X}(0, \pi)=1$ e Tra $J \bar{X}(0, \pi)=-2$, a singularidade $(0, \pi)$ é do tipo nó atrator. Reunindo tais informações e aplicando um blowing down, concluímos que o retrato de fase de $X$ é dado por: 

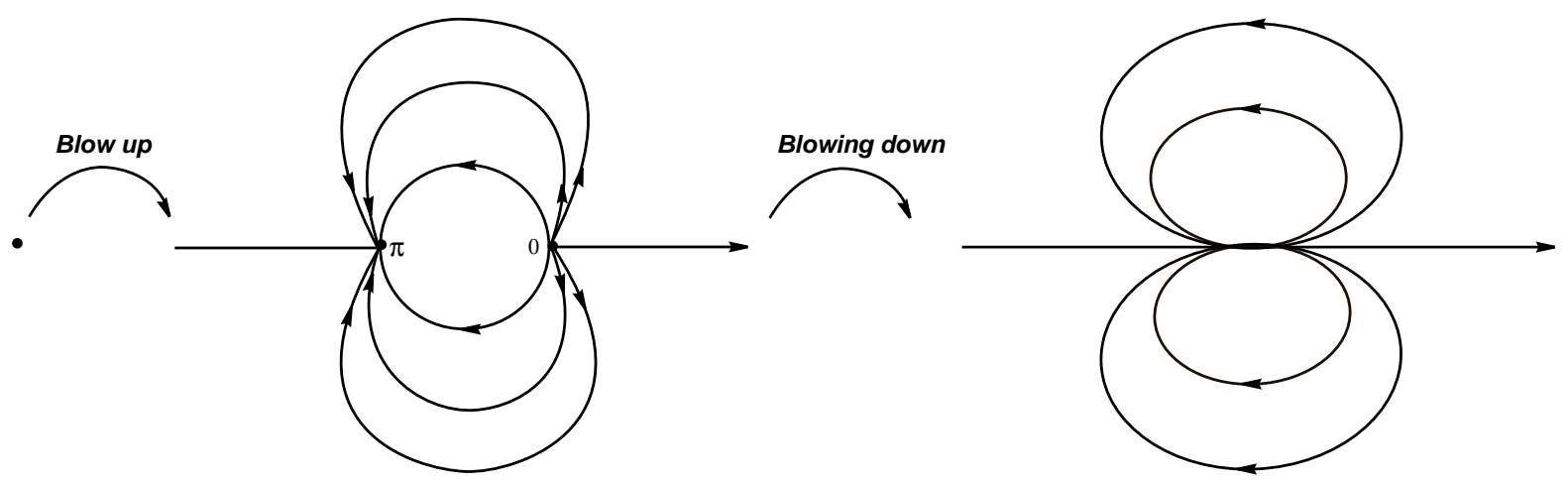

Figura 1.11: Exemplo de blow up polar

\subsubsection{Blow up direcional}

Blow up na direção $x$

Seja $X=(P(x, y), Q(x, y))$ um campo de vetores em $\mathbb{R}^{2}$, com singularidade isolada em $(0,0)$. Se $x=u$ e $y=u v$, temos

$$
\left\{\begin{array} { l } 
{ \dot { x } = \dot { u } } \\
{ \dot { y } = \dot { u } v + \dot { v } u }
\end{array} \quad \text { e } \quad \left\{\begin{array}{l}
\dot{u}=\dot{x} \\
\dot{v}=\frac{\dot{y}-\dot{u} v}{u}
\end{array}\right.\right.
$$

ou seja,

$$
\left\{\begin{array}{l}
\dot{u}=P(u, u v) \\
\dot{v}=\frac{Q(u, u v)-v P(u, u v)}{u}
\end{array}\right.
$$

Chame o campo $\tilde{X}(u, v)=\left(\tilde{X}_{u}, \tilde{X}_{v}\right)$, onde $\tilde{X}_{u}=P(u, u v)$ e $\tilde{X}_{v}=\frac{Q(u, u v)-v P(u, u v)}{u}$.

Então seja $\kappa$ o maior inteiro tal que $u^{\kappa} \mid \tilde{X}_{u}$ e $u^{\kappa} \mid \tilde{X}_{v}$.

Defina $\bar{X}=\left(\bar{X}_{u}, \bar{X}_{v}\right)=\frac{\tilde{X}(u, v)}{u^{\kappa}}$. A singularidade $(0,0)$ é levada em $u=0$ e, para $u=0$ as singularidades de $\bar{X}$ são determinadas pelas soluções de $\bar{X}_{v}=0$.

\section{Blow up na direção y}

Seja $X=(P(x, y), Q(x, y))$ um campo de vetores polinomial com singularidade isolada em $(0,0)$. Se $x=u v$ e $y=v$, então 


$$
\left\{\begin{array} { l } 
{ \dot { x } = \dot { u } v + \dot { v } u } \\
{ \dot { y } = \dot { v } }
\end{array} \text { e } \left\{\begin{array}{l}
\dot{u}=\frac{\dot{x}-\dot{v} u}{v} \\
\dot{v}=\dot{y}
\end{array}\right.\right.
$$

Defina

$$
\tilde{X}(u, v)=\left(\tilde{X}_{u}, \tilde{X}_{v}\right)=\left(\frac{P(u v, v)-u Q(u v, v)}{v}, Q(u v, v)\right)
$$

e seja $\kappa$ o maior inteiro tal que $v^{\kappa} \mid \tilde{X}_{u}$ e $v^{\kappa} \mid \tilde{X}_{v}$. Defina $\bar{X}=\left(\bar{X}_{u}, \bar{X}_{v}\right)=\frac{\tilde{X}(u v, v)}{v^{\kappa}}$

Neste caso, a singularidade $(0,0)$ é levada para a curva $v=0$ e em $v=0$ as singularidades são determinadas pelas soluções de $\bar{X}_{u}=0$.

Vejamos essa técnica em exemplos:

Exemplo 1.3.3. Considere o campo $X=\left(x^{2}-2 x y, y^{2}-2 x y\right)$ com singularidade isolada em $(0,0)$.

Aplicando blow up na direção $x$ em $(0,0), x=u$ e $y=u v$ encontramos:

$$
\tilde{X}=\left(\tilde{X}_{u}, \tilde{X}_{v}\right)=\left(u^{2}-2 u^{2} v, 3 u v^{2}-3 u v\right)
$$

Como $\kappa=1$ é o maior inteiro tal que $u^{\kappa} \mid \tilde{X}_{u}$ e $u^{\kappa} \mid \tilde{X}_{v}$, temos

$$
\bar{X}=\frac{\tilde{X}}{u}=\left(\bar{X}_{u}, \bar{X}_{v}\right)=\left(u-2 u v, 3 v^{2}-3 v\right)
$$

Em $u=0$, temos $v=0$ e $v=1$ como raízes de $\bar{X}_{v}=0$. Como

$$
J \bar{X}(u, v)=\left[\begin{array}{cc}
1-2 v & -2 u \\
0 & 6 v-3
\end{array}\right] \quad \text { e } \begin{array}{r}
\operatorname{det} J \bar{X}(0,0)=-3 \\
\operatorname{det} J \bar{X}(0,1)=-3,
\end{array}
$$

as singularidades $(0,0)$ e $(0,1)$ são do tipo sela.

Agora, aplicamos um blow up na direção $y, x=u v$ e $y=v$ obtemos:

$$
\tilde{X}=\left(\tilde{X}_{u}, \tilde{X}_{v}\right)=\left(3 u^{2} v-3 u v, v^{2}-2 u v^{2}\right)
$$

Neste caso, $\bar{X}=\frac{\tilde{X}}{v}=\left(\bar{X}_{u}, \bar{X}_{v}\right)=\left(3 u^{2}-3 u, v-2 u v\right)$.

Em $v=0$ temos $u=0$ e $u=1$ como raízes de $\tilde{X}_{u}=0$. Sendo

$$
J \bar{X}(u, v)=\left[\begin{array}{cc}
6 u-3 & 0 \\
-2 v & 1-2 u
\end{array}\right] \text { e } \begin{array}{r}
\operatorname{det} J \bar{X}(0,0)=-3 \\
\operatorname{det} J \bar{X}(1,0)=-3
\end{array}
$$




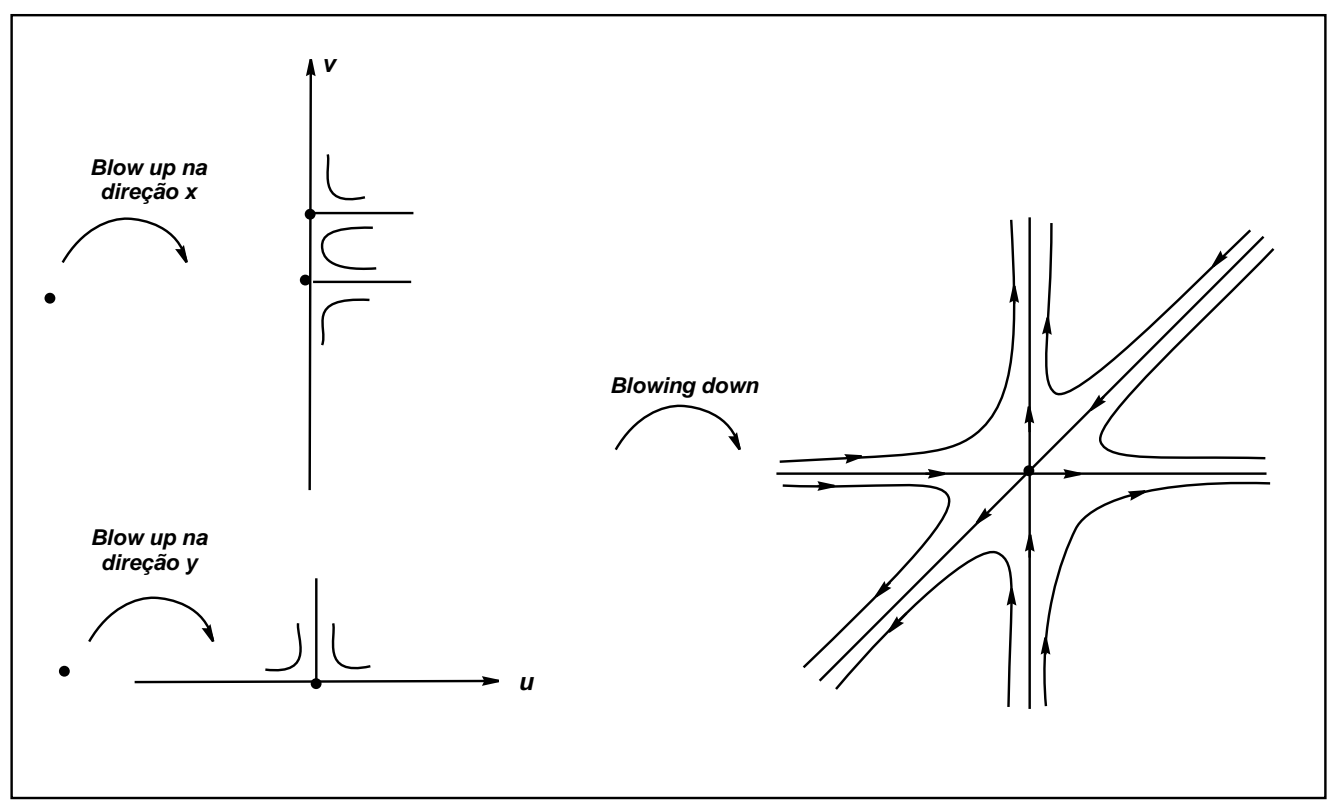

Figura 1.12: Exemplo de blow up direcional

Concluímos que $(0,0)$ e $(1,0)$ são singularidades do tipo sela.

Observe o esboço do retrato de fase de $X$ na Figura 1.12. Note que este é exatamente o mesmo retrato obtido pelo blow up polar no exemplo 1.3.1.

O exemplo 1.3.3 leva-nos a perguntar: Qual é a relação entre blow up polar e direcional?

A seguir, esta pergunta será respondida.

\subsubsection{Relação entre blow up polar e blow up direcional}

Seja $F: \mathbb{R} \times\left(-\frac{\pi}{2}, \frac{\pi}{2}\right) \rightarrow \mathbb{R}^{2}$ dada por $F(r, \theta)=(r \cos \theta, \tan \theta)$.

Temos que $J F(r, \theta)=\left[\begin{array}{cc}\cos \theta & -r \operatorname{sen} \theta \\ 0 & \sec ^{2} \theta\end{array}\right]$. Logo, $\operatorname{det} J F(r, \theta)=\sec \theta \neq 0$

Portanto, $F(r, \theta)$ é difeomorfismo.

Além disso:

(i) F leva o semi-círculo $\left\{(r, \theta) ; r=0\right.$ e $\left.-\frac{\pi}{2}<\theta<\frac{\pi}{2}\right\}$ sobre o eixo $O_{y}$ sendo que para $\theta=0$ temos $F(r, 0)=(0,0) \in O_{x y}$.

(ii) $J F(0,0)=\left[\begin{array}{ll}1 & 0 \\ 0 & 1\end{array}\right]$, então $F$ mantém a parte linear de seu campo. 
(iii) Finalmente, $F(r, \theta)=\left(x, \frac{y}{x}\right)$, portanto $F^{-1}(u, v)=F^{-1}\left(x, \frac{y}{x}\right)=(r, \theta)$, pois:

$$
\left\{\begin{array} { l } 
{ u = r \operatorname { c o s } \theta } \\
{ v = \operatorname { t a n } \theta }
\end{array} \Rightarrow \left\{\begin{array}{l}
u=r \cos \theta=x \\
u v=r \operatorname{sen} \theta=y
\end{array}\right.\right.
$$

Considere então, o campo do exemplo 1.3.3.

Quando aplicamos o blow up na direção $x$, obtivemos as singularidades $(0,1)$ e $(0,0)$ que são do tipo sela.

Logo: $\left\{\begin{array}{l}(u, v) \rightarrow(r, \theta) \\ (0,0) \rightarrow(0,0) \\ (0,1) \rightarrow\left(0, \frac{\pi}{4}\right)\end{array}\right.$

Por simetria, temos $\pi$ e $\frac{5 \pi}{4}$ singularidades do tipo sela, como podemos observar na Figura 1.13.

Blow Up na direção $x$

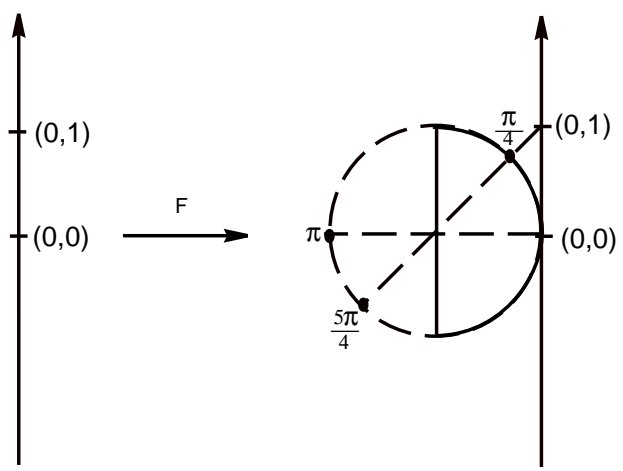

Figura 1.13: Relação entre blow up na direção $x$ e blow up polar.

Seja $G:(0, \pi) \times \mathbb{R} \rightarrow \mathbb{R}^{2}$ dada por $G(\theta, r)=(\operatorname{cotg} \theta, r \operatorname{sen} \theta)$.

Temos que $J G(\theta, r)=\left[\begin{array}{cc}-\operatorname{cossec} \theta & 0 \\ r \cos \theta & \operatorname{sen} \theta\end{array}\right] \Rightarrow \operatorname{det} J G(\theta, r)=-\operatorname{cossec} \theta \neq 0$

Portanto, $G(\theta, r)$ é difeomorfismo.

Além disso: 
(i) $G$ leva o semi-círculo $\{(\theta, r) ; r=0$ e $0<\theta<\pi\}$ sobre o eixo $O_{x}$, sendo que para $\theta=\frac{\pi}{2} \operatorname{temos} G\left(\frac{\pi}{2}, 0\right)=(0,0) \in O_{x y}$.

(ii) $J G\left(\frac{\pi}{2}, 0\right)=\left[\begin{array}{cc}-1 & 0 \\ 0 & 1\end{array}\right]$, então $G$ mantém a parte linear de seu campo.

(iii) Finalmente, $G(\theta, r)=\left(\frac{x}{y}, y\right)$, portanto $G^{-1}(u, v)=G^{-1}\left(\frac{x}{y}, y\right)=(r, \theta)$, pois:

$$
\left\{\begin{array} { l } 
{ u = \operatorname { c o t g } \theta } \\
{ v = r \operatorname { s e n } \theta }
\end{array} \Rightarrow \left\{\begin{array}{l}
u v=r \cos \theta=x \\
v=r \operatorname{sen} \theta=y
\end{array}\right.\right.
$$

Considere então o campo do exemplo 1.3.3.

Quando aplicamos o blow up na direção $y$, obtivemos as singularidades $(0,1)$ e $(0,0)$ que são do tipo sela.

Logo: $\left\{\begin{aligned}(u, v) & \rightarrow(\theta, r) \\ (0,0) & \rightarrow\left(\frac{\pi}{2}, 0\right) \\ (1,0) & \rightarrow\left(\frac{\pi}{4}, 0\right)\end{aligned}\right.$

Por simetria, temos $\frac{3 \pi}{2}$ e $\frac{5 \pi}{4}$ singularidades do tipo sela, como podemos observar na Figura 1.14.

Blow Up na direção y

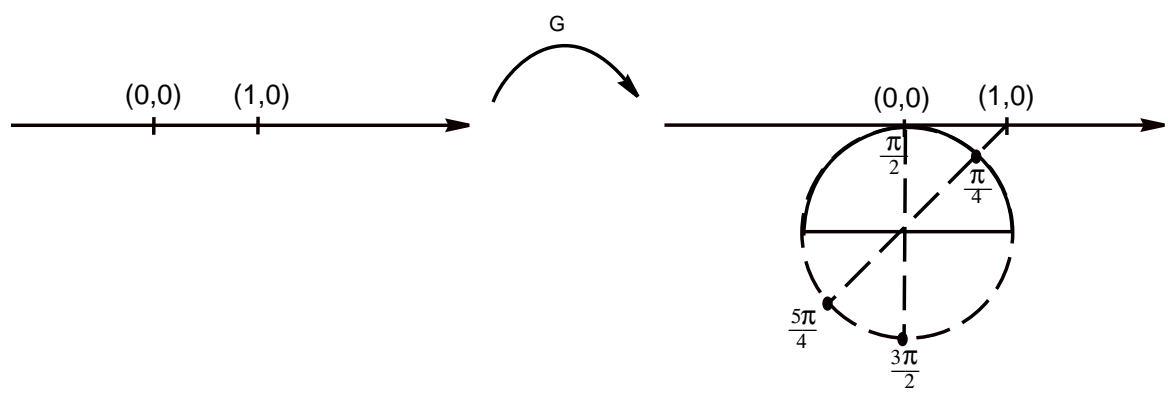

Figura 1.14: Relação entre blow up na direção y e blow up polar.

A escolha por uma das técnicas, blow up polar ou blow up direcional, é opcional. Porém, 
em geral, para blow ups sucessivos é mais conveniente utilizar o blow up direcional, como observamos nos próximos exemplos.

Exemplo 1.3.4. Considere o campo $X=\left(y^{3}, x+y^{2}+y^{3}\right)$ com singularidade isolada em $(0,0)$.

Vamos aplicar blow up na direção $x$ em $(0,0)$.

Através da mudança de coordenadas $x=u$ e $y=u v$ encontramos:

$$
\tilde{X}=\left(\tilde{X}_{u}, \tilde{X}_{v}\right)=\left(u^{3} v^{3}, 1+u v^{2}+u^{2} v^{3}-u^{2} v^{4}\right) .
$$

Neste caso, não existe $\kappa$ tal que $u^{\kappa} \mid \tilde{X}_{u}$ e $u^{\kappa} \mid \tilde{X}_{v}$.

Por isso, $\bar{X}=\tilde{X}$.

Ao longo de $u=0$, temos $\tilde{X}_{v}=1$, portanto, não existe singularidade na direção de $x$.

Aplicando um blow up na direção $y, x=u v$ e $y=v$ obtemos:

$$
\tilde{X}=\left(\tilde{X}_{u}, \tilde{X}_{v}\right)=\left(v^{2}-u^{2}-u v-u v^{2}, u v+v^{2}+v^{3}\right)
$$

Observe que não existe $\kappa>0$, tal que $v^{\kappa} \mid \tilde{X}_{u}$ e $v^{\kappa} \mid \tilde{X}_{v}$. Portanto, $\bar{X}=\tilde{X}$.

Em $v=0$ temos $u=0$ como solução de $\tilde{X}_{u}=0$ e ainda,

$$
J \bar{X}(p)=\left[\begin{array}{cc}
-2 u-v-v^{2} & 2 v-u-2 u v \\
v & u+2 v+3 v^{3}
\end{array}\right] \Rightarrow J \bar{X}(0,0)=\left[\begin{array}{ll}
0 & 0 \\
0 & 0
\end{array}\right]
$$

Como det $J \bar{X}(0,0)=0$, temos que $(0,0)$ é uma singularidade não hiperbólica. Aplicando um novo blow up na direção de $v$ em $(0,0), u=x y$ e $v=y$ obtemos:

$$
\tilde{X}=\left(\tilde{X}_{x}, \tilde{X}_{y}\right)=\left(y-2 x^{2} y-2 x y-2 x y^{2}, x y^{2}+y^{2}+y^{3}\right)
$$

Então $\kappa=1$ é o maior inteiro tal que $y^{\kappa} \mid \tilde{X}_{x}$ e $y^{\kappa} \mid \tilde{X}_{y}$.

Assim, $\bar{X}=\frac{\tilde{X}}{y}=\left(\bar{X}_{x}, \bar{X}_{y}\right)=\left(1-2 x^{2}-2 x-2 x y, x y+y+y^{2}\right)$.

Em $y=0$, as soluções de $\bar{X}_{x}=0$ são $\frac{-2-\sqrt{12}}{4}$ e $\frac{-2+\sqrt{12}}{4}$.

O Jacobiano de $\bar{X}$ é dado por: 


$$
J \bar{X}(x, y)=\left[\begin{array}{cc}
-4 x-2 & -2 x \\
y & x+2 y+1
\end{array}\right] \Rightarrow\left\{\begin{array}{c}
\operatorname{det} J \bar{X}\left(0, \frac{-2-\sqrt{12}}{4}\right)=\frac{\sqrt{12}}{2}-3<0 \\
\operatorname{det} J \bar{X}\left(0, \frac{-2+\sqrt{12}}{4}\right)=-\frac{\sqrt{12}}{2}-3<0
\end{array}\right.
$$

Portanto, as singularidades $\left(0, \frac{-2-\sqrt{12}}{4}\right)$ e $\left(0, \frac{-2+\sqrt{12}}{4}\right)$ são do tipo sela.

Observe na Figura 1.15 as etapas para obtermos um esboço do retrato de fase de $X$.
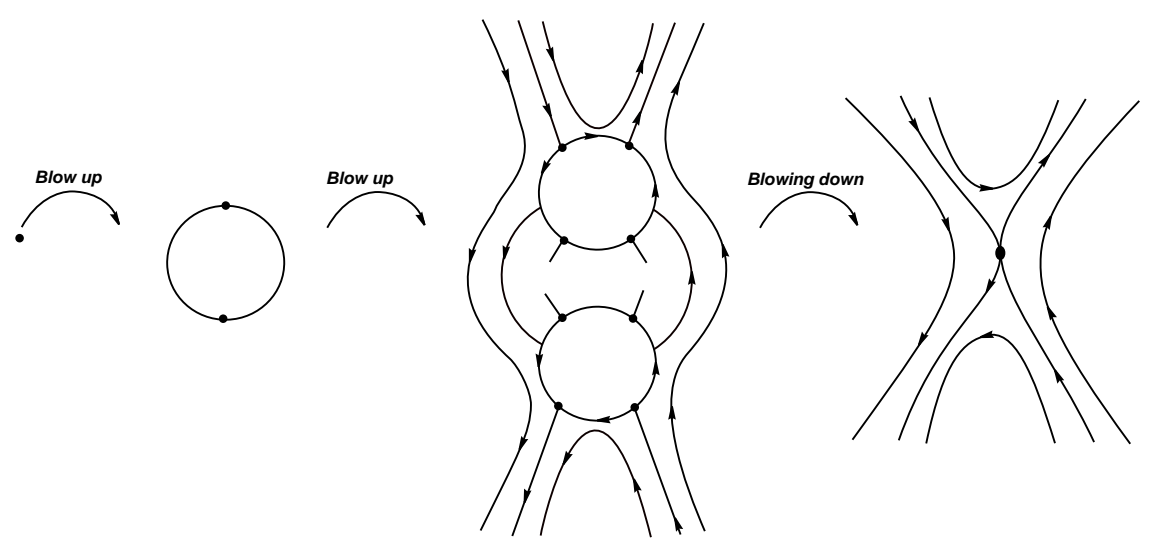

Figura 1.15: Exemplo de blow ups direcionais sucessivos

Exemplo 1.3.5. Considere o campo $X=\left(-y^{3}, x+2 y^{2}\right)$ com singularidade isolada em $(0,0)$.

Aplicando blow up na direção $x$ em $(0,0), x=u, y=u v$ encontramos:

$$
\tilde{X}=\left(\tilde{X}_{u}, \tilde{X}_{v}\right)=\left(-u^{3} v^{3}, 1+2 u v^{2}+u^{2} v^{4}\right)
$$

Neste caso, não existe $\kappa$, tal que $u^{\kappa} \mid \tilde{X}_{u}$ e $u^{\kappa} \mid \tilde{X}_{v}$.

Então, $\bar{X}=\tilde{X}$.

Fazendo $u=0$ temos $\tilde{X}_{v}=1 \mathrm{e}$, portanto, não existe singularidade na direção de $x$.

Aplicando blow up na direção $y, x=u v, y=v$, obtemos: 


$$
\tilde{X}=\left(\tilde{X}_{u}, \tilde{X}_{v}\right)=\left(-v^{2}-u^{2}-2 u v, u v+2 v^{2}\right)
$$

Observe que não existe $\kappa>0$ tal que $v^{\kappa} \mid \tilde{X}_{u}$ e $v^{\kappa} \mid \tilde{X}_{v}$. Portanto, $\bar{X}=\tilde{X}$.

Em $v=0$, temos $u=0$ como solução de $\tilde{X}_{u}=0$ e

$$
J \bar{X}(p)=\left[\begin{array}{cc}
-2 u-2 v & 2 v-2 u \\
v & u+4 v
\end{array}\right] \Rightarrow J \bar{X}(0,0)=\left[\begin{array}{ll}
0 & 0 \\
0 & 0
\end{array}\right]
$$

Como det $J \bar{X}(0,0)=0$, temos que $(0,0)$ é uma singularidade não hiperbólica. Aplicando novo blow up na direção de $y$ em $(0,0), u=x y$ e $v=y$ obtemos:

$$
\tilde{X}=\left(\tilde{X}_{x}, \tilde{X}_{y}\right)=\left(-y-4 x y-2 x y^{2}, x y^{2}+2 y^{2}\right)
$$

Nesse caso, $\kappa=1$ é o maior inteiro, tal que $y^{\kappa} \mid \tilde{X}_{x}$ e $y^{\kappa} \mid \tilde{X}_{y}$.

Assim, $\bar{X}=\frac{\tilde{X}}{y}=\left(\bar{X}_{x},=\bar{X}_{y}\right)=\left(-1-2 x^{2}-4 x, x y+2 y\right)$

Em $y=0$, as soluções de $\bar{X}_{x}=0$ são $\frac{-2-\sqrt{2}}{2}$ e $\frac{-2+\sqrt{2}}{2}$.

O jacobiano de $\bar{X}$ é dado por:

$$
J \bar{X}(x, y)=\left[\begin{array}{cc}
-4 x-4 & 0 \\
y & x+2
\end{array}\right]
$$

$\Rightarrow\left\{\begin{array}{l}\operatorname{det} J \bar{X}\left(0, \frac{-2-\sqrt{2}}{2}\right)=2 \sqrt{12}-2>0 e \operatorname{Tra} \bar{X}\left(0, \frac{-2-\sqrt{2}}{2}\right)=\frac{3 \sqrt{2}}{2}+1>0 \\ \operatorname{det} J \bar{X}\left(0, \frac{-2+\sqrt{2}}{2}\right)=-2 \sqrt{2}-2<0\end{array}\right.$

Portanto, a singularidade $\left(0, \frac{-2-\sqrt{2}}{2}\right)$ é do tipo nó repulsor e $\left(0, \frac{-2+\sqrt{2}}{2}\right)$ é do tipo sela.

Observe na Figura 1.16 as etapas para obtermos um esboço do retrato de fase de $X$. 


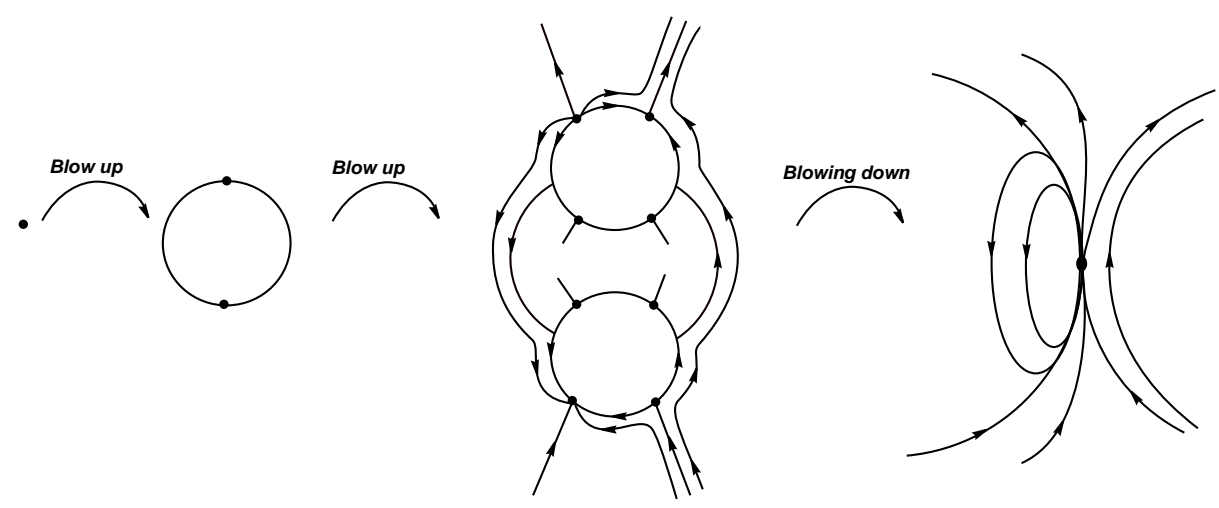

Figura 1.16: Exemplo de blow ups direcionais sucessivos

\subsection{Compactificação de Poincaré}

Seja $X$ um campo de vetores definido em $\mathbb{R}^{2}$ tal que $X(x, y)=(P(x, y), Q(x, y))$, onde $P$ e $Q \in \mathbb{R}[x, y]$ e o máximo dos graus de $P$ e $Q$ é $n$. Nesta seção, mostramos como obter um campo canonicamente induzido por $X$ na esfera $\mathbb{S}^{2}$. Primeiramente, obtemos um campo induzido nos hemisférios superior e inferior da esfera por projeção central. Em seguida, mostramos como proceder para estender o campo induzido a $\mathbb{S}^{2}$ e a relação existente entre $\mathbb{S}^{1}$ e o infinito de $\mathbb{R}^{2}$. Essa técnica é conhecida como compactificação de Poincaré. Ela permite o estudo local de $X$ nos pontos críticos do infinito.

\subsubsection{Induzindo um campo de vetores na esfera}

Seja $y=\left(y_{1}, y_{2}, y_{3}\right)$ um ponto arbitrário em $\mathbb{R}^{3}$. Identifique $\mathbb{R}^{2}$ com o subespaço afim $\left\{y \in \mathbb{R}^{3} \mid y_{3}=1\right\}$ e considere a esfera $\mathbb{S}^{2}=\left\{y \in \mathbb{R}^{3} \mid\|y\|=1\right\}$, a qual chamamos de esfera de Poincaré; ela é tangente a $\mathbb{R}^{2}$ no ponto $(0,0,1)$. Sejam $H_{+}=\left\{y \in \mathbb{S}^{2}: y_{3}>0\right\}$ (hemisfério norte), $H_{-}=\left\{y \in \mathbb{S}^{2}: y_{3}<0\right\}$ (hemisfério sul) e $\mathbb{S}^{1}=\left\{y \in \mathbb{S}^{2}: y_{3}=0\right\}$ (o equador). Considerando a projeção das trajetórias de $X$ em $\mathbb{R}^{2}$ em $\mathbb{S}^{2}$, por meio da projeção central, encontramos um fluxo em $\mathbb{S}^{2}$. Vejamos como expressar tal projeção analiticamente.

Pela projeção central de $\mathbb{R}^{2}$ em $\mathbb{S}^{2}$, para cada ponto $P=\left(x_{1}, x_{2}, 1\right)$ de $\mathbb{R}^{2}$, temos dois pontos correspondentes em $\mathbb{S}^{2}$, um no hemisfério superior e outro no hemisfério inferior da esfera. De fato, definamos as funções:

$$
f^{+}(x)=\frac{\left(x_{1}, x_{2}, 1\right)}{\Delta(x)} \quad f^{-}(x)=-\frac{\left(x_{1}, x_{2}, 1\right)}{\Delta(x)}
$$




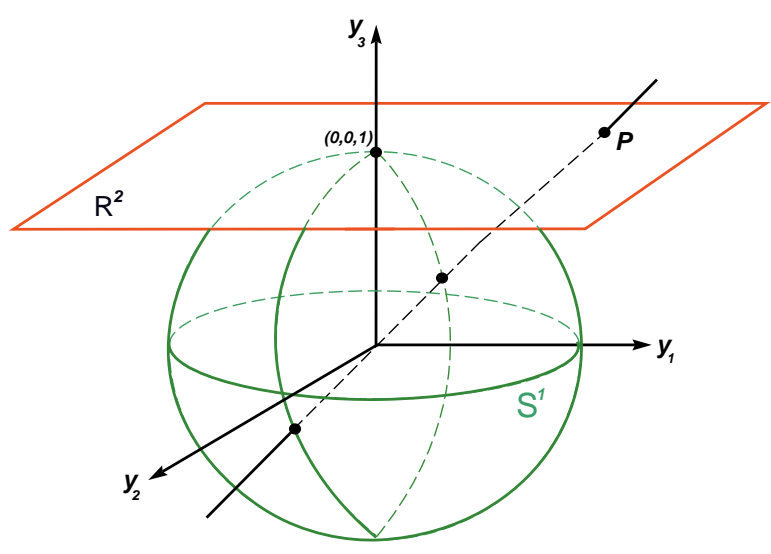

Figura 1.17: Projeção Central

onde $\Delta(x)=\left(x_{1}^{2}+x_{1}^{2}+1\right)^{1 / 2}$. Note que, $f^{+}(x)$ (respectivamente $f^{-}(x)$ ) é a interseção do hemisfério norte (respectivamente sul) de $\mathbb{S}^{2}$ com a reta que passa por $P$ e pelo centro de $\mathbb{S}^{2}$.

Dessa maneira, obtemos do campo vetorial $X$ um campo induzido $\bar{X}$ em $\mathbb{S}^{2} \backslash \mathbb{S}^{1}$.

O campo vetorial induzido em $H_{+}$é dado por $\bar{X}(y)=D f^{+}(x) X(x)$, onde $y=f^{+}(x)$ e, em $H_{-}$é $\bar{X}(y)=D f^{-}(x) X(x)$, onde $y=f^{-}(x)$.

Vejamos como estender o campo vetorial induzido $\bar{X}$ de $\mathbb{S}^{2} \backslash \mathbb{S}^{1}$ para $\mathbb{S}^{2}$. O campo estendido é chamado compactificação de Poincaré do campo vetorial $X$ em $\mathbb{R}^{2}$ e é denotado por $\rho(x)$.

Definição 1.4.1. Sejam $X$ e $Y$ dois campos vetoriais em $\mathbb{R}^{2}$. Dizemos que $\rho(X)$ e $\rho(Y)$ são topologicamente equivalentes se existe um homeomorfismo $h: \mathbb{S}^{2} \rightarrow \mathbb{S}^{2}$ que leva órbitas de $\rho(X)$ em órbitas de $\rho(Y)$, preservando ou revertendo simultaneamente o sentido de todas as órbitas.

Sendo $\mathbb{S}^{2}$ uma variedade diferenciável, considere os abertos $U_{k}=\left\{y \in \mathbb{S}^{2} \mid y_{k}>0, k=\right.$ $1,2,3\}$ e $V_{k}=\left\{y \in \mathbb{S}^{2} \mid y_{k}<0, k=1,2,3\right\}$ e as cartas locais $\phi_{k}: U_{k} \rightarrow \mathbb{R}^{2}$ e $\psi_{k}: V_{k} \rightarrow \mathbb{R}^{2}$ definidas por:

$$
\phi_{k}(y)=\frac{\left(y_{m}, y_{n}\right)}{y_{k}} \quad \psi_{k}(y)=\frac{\left(y_{m}, y_{n}\right)}{y_{k}}
$$

$m, n, k=1,2,3 ; m<n$ e $m, n \neq k$.

Denotamos por $z=\left(z_{1}, z_{2}\right)$ o valor de $\phi_{k}(y)$ ou $\psi_{k}(y)$, para algum $k$. Dessa forma, 
$\left(z_{1}, z_{2}\right)$ desempenha diferentes papéis de acordo com a carta local que consideramos. Os pontos de $\mathbb{S}^{1}$ têm $z_{2}=0$ nas cartas locais.

A seguir, faremos a construção detalhada da expressão do campo compactificado, $\bar{X}$, nas cartas $U_{1}$ e $V_{1}$.

- $\operatorname{Em} U_{1}$

Se $y \in U_{1}, y=f^{+}(x)$ e $\bar{X}(y)=D f^{+}(x) X(x)$ então

$$
D \phi_{1}(y) \bar{X}(y)=D \phi_{1}(y) \circ D f^{+} X(x)=D\left(\phi_{1} \circ f^{+}\right)(x) X(x) .
$$

De (1.4), segue que:

$$
\left(\phi_{1} \circ f^{+}\right)(x)=\phi_{1}\left(\frac{\left(x_{1}, x_{2}, 1\right)}{\Delta(x)}\right)=\left(\frac{x_{2}}{x_{1}}, \frac{1}{x_{1}}\right)=\left(z_{1}, z_{2}\right)
$$

$\log \mathrm{O}$

$$
D\left(\phi_{1} \circ f^{+}\right)(x) X(x)=\left[\begin{array}{cc}
-\frac{x_{2}}{x_{1}^{2}} & \frac{1}{x_{1}} \\
-\frac{1}{x_{1}^{2}} & 0
\end{array}\right]\left[\begin{array}{l}
P\left(x_{1}, x_{2}\right) \\
Q\left(x_{1}, x_{2}\right)
\end{array}\right]
$$

Então, se $\left.\bar{X}\right|_{U_{1}}$ denota o campo vetorial $D\left(\phi_{1} \circ f^{+}\right)(x) X(x)$ temos que

$$
\left.\bar{X}\right|_{U_{1}}=\left(\frac{1}{x_{1}}\right)^{2}\left[x_{1} Q\left(x_{1}, x_{2}\right)-x_{2} P\left(x_{1}, x_{2}\right),-P\left(x_{1}, x_{2}\right)\right] .
$$

Por outro lado, de (1.5) temos

$$
\left(\phi_{1} \circ f^{+}\right)(x)=\phi_{1}\left(f^{+}(x)\right)=\phi_{1}(y)=\left(\frac{y_{2}}{y_{1}}, \frac{y_{3}}{y_{1}}\right)=\left(z_{1}, z_{2}\right)
$$

e de (1.6) e (1.7) obtemos: $\left(x_{1}, x_{2}\right)=\left(\frac{y_{1}}{y_{3}}, \frac{y_{2}}{y_{3}}\right)$. Desta forma, encontramos:

$$
\left.\bar{X}\right|_{U_{1}}=\left(\frac{y_{3}}{y_{1}}\right)^{2}\left[\frac{y_{1}}{y_{3}} Q\left(\frac{y_{1}}{y_{3}}, \frac{y_{2}}{y_{3}}\right)-\frac{y_{2}}{y_{3}} P\left(\frac{y_{1}}{y_{3}}, \frac{y_{2}}{y_{3}}\right),-P\left(\frac{y_{1}}{y_{3}}, \frac{y_{2}}{y_{3}}\right)\right] .
$$

- $\operatorname{Em} V_{1}$ 
Se $y \in V_{1}, y=f^{-}(x)$ e $\bar{X}(y)=D f^{-}(x) X(x)$.

De (1.4) temos:

$$
\left(\phi_{1} \circ f^{-}\right)(x)=\phi_{1}\left(\frac{-\left(x_{1}, x_{2}, 1\right)}{\Delta(x)}\right)=\left(\frac{-x_{2}}{-x_{1}}, \frac{-1}{-x_{1}}\right)=\left(z_{1}, z_{2}\right)
$$

e de $(1.5)$

$$
\left(\phi_{1} \circ f^{-}\right)(x)=\phi_{1}\left(f^{-}(x)\right)=\phi_{1}(y)=\left(\frac{y_{2}}{y_{1}}, \frac{y_{3}}{y_{1}}\right)=\left(z_{1}, z_{2}\right) .
$$

Logo, a expressão obtida para $\left.\bar{X}\right|_{V_{1}}$ é a mesma que a encontrada para $\left.\bar{X}\right|_{U_{1}}$.

Procedendo de maneira análoga obtemos as expressões:

$$
\left.\bar{X}\right|_{U_{2}}=\left.\bar{X}\right|_{V_{2}}=\left(\frac{y_{3}}{y_{2}}\right)^{2}\left[\frac{y_{2}}{y_{3}} P\left(\frac{y_{1}}{y_{3}}, \frac{y_{2}}{y_{3}}\right)-\frac{y_{1}}{y_{3}} Q\left(\frac{y_{1}}{y_{3}}, \frac{y_{2}}{y_{3}}\right),-Q\left(\frac{y_{1}}{y_{3}}, \frac{y_{2}}{y_{3}}\right)\right]
$$

$\mathrm{e}$

$$
\left.\bar{X}\right|_{U_{3}}=\left.\bar{X}\right|_{V_{3}}=\left[P\left(\frac{y_{1}}{y_{3}}, \frac{y_{2}}{y_{3}}\right), Q\left(\frac{y_{1}}{y_{3}}, \frac{y_{2}}{y_{3}}\right)\right] .
$$

Note que os pontos do infinito de $\mathbb{R}^{2}$ (dois para cada direção) estão em correspondência um-a-um com os pontos em $\mathbb{S}^{1}$. Além disso, não é possível estender $\left.\bar{X}\right|_{U_{i}}$ e $\left.\bar{X}\right|_{V_{i}}, i=1,2,3$ para o equador $\mathbb{S}^{1}$, pois obtemos campos de vetores induzidos em $U_{i}$ e $V_{i}$, com $i=1,2,3$, os quais são definidos e diferenciáveis em todos os pontos, exceto para $y_{3}=0$, isto é, exceto em $\mathbb{S}^{1}$. No entanto, temos o seguinte teorema:

Teorema 1.4.2. Considere $f^{+}$e $f^{-}$as projeções centrais definidas em (1.4). O campo induzido no complementar do equador, $\mathbb{S}^{2} \backslash \mathbb{S}^{1}$ por meio das diferenciais de $f^{+}$e $f^{-}$pode ser estendido analiticamente $a \mathbb{S}^{2}$ depois de multiplicado pelo fator $y_{3}^{n-1}$ de tal forma que o equador seja invariante pelo campo.

\section{Demonstração:}

Multiplicando (1.8), (1.11), (1.12) pelo fator $y_{3}^{n-1}$, obtemos um novo campo que é diferenciável em todo $U_{i}$ e $V_{i}$, com $i=1,2,3$. Essa multiplicação elimina fatores de $y_{3}$ que aparecem nos denominadores. Observe que o fator $y_{3}^{n-1}$ depende apenas do grau do sistema $X$ original. 
Por propósitos analíticos, antes de multiplicarmos o campo obtido pelo fator $y_{3}^{n-1}$, vamos expressá-lo em termos da variável $z=\left(z_{1}, z_{2}\right)$.

Para o caso em que $y \in U_{1}$ ou $y \in V_{1}$, de (1.7), segue que: $\frac{y_{1}}{y_{3}}=\frac{1}{z_{2}}, \frac{y_{2}}{y_{3}}=\frac{z_{1}}{z_{2}}$.

Assim,

$$
\left.\bar{X}\right|_{U_{1}}=\left.\bar{X}\right|_{V_{1}}=z_{2}\left[Q\left(\frac{1}{z_{1}}, \frac{z_{1}}{z_{2}}\right)-z_{1} P\left(\frac{1}{z_{2}}, \frac{z_{1}}{z_{2}}\right),-z_{2} P\left(\frac{1}{z_{2}}, \frac{z_{1}}{z_{2}}\right)\right] .
$$

Temos ainda:

$$
\Delta(z)=\left(z_{1}^{2}+z_{2}^{2}+1\right)^{\frac{1}{2}}=\left(\frac{y_{2}^{2}}{y_{1}^{2}}+\frac{y_{3}^{2}}{y_{1}^{2}}+1\right)^{\frac{1}{2}}=\frac{\left(y_{2}^{2}+y_{3}^{2}+y_{1}^{2}\right)^{\frac{1}{2}}}{\left|y_{1}\right|}=\frac{\|y\|}{\left|y_{1}\right|}=\frac{1}{\left|y_{1}\right|} .
$$

Agora em $U_{1}, y_{1}>0$, e como $\frac{y_{3}}{y_{1}}=z_{2}$, segue que $y_{3}$ terá sempre o mesmo sinal que $z_{2}$. Então, $y_{3}=\frac{z_{2}}{\Delta(z)}$. Finalmente, multiplicando (1.13) por $y_{3}^{n-1}=\frac{z_{2}^{n-1}}{\Delta(z)^{n-1}}$ obtemos a expressão final para o campo em $U_{1}$ :

$$
\left.\bar{X}\right|_{U_{1}}=\frac{z_{2}^{n}}{\Delta(z)^{n-1}}\left[Q\left(\frac{1}{z_{1}}, \frac{z_{1}}{z_{2}}\right)-z_{1} P\left(\frac{1}{z_{2}}, \frac{z_{1}}{z_{2}}\right),-z_{2} P\left(\frac{1}{z_{2}}, \frac{z_{1}}{z_{2}}\right)\right] .
$$

De forma semelhante, obtemos as expressões de $\bar{X}$ para $y \in U_{2}$ e $y \in U_{3}$, as quais são dadas por (1.15) e (1.16) respectivamente:

$$
\begin{gathered}
\frac{z_{2}^{n}}{\Delta(z)^{n-1}}\left[P\left(\frac{z_{1}}{z_{2}}, \frac{1}{z_{2}}\right)-z_{1} Q\left(\frac{z_{1}}{z_{2}}, \frac{1}{z_{2}}\right),-z_{2} Q\left(\frac{1}{z_{2}}, \frac{z_{1}}{z_{2}}\right)\right] \\
\frac{1}{\Delta(z)^{n-1}}\left[P\left(z_{1}, z_{2}\right), Q\left(z_{1}, z_{2}\right)\right] .
\end{gathered}
$$

Para o caso em que $y \in V_{i}, i=1,2,3$, as expressões obtidas são (1.14), (1.15), (1.16) exceto pelo seguinte fato: em $V_{1}, y_{1}<0$ e então $y_{3}$ e $z_{2}$ têm sinais contrários. Desta forma, $y_{3}=\frac{-z_{2}}{\Delta(z)}$. Logo, multiplicamos (1.13) por $y_{3}^{n-1}=(-1)^{n-1} \frac{z_{2}^{n-1}}{\Delta(z)^{n-1}}$ e obtemos:

$$
\left.\bar{X}\right|_{V_{1}}=(-1)^{n-1} \frac{z_{2}^{n}}{\Delta(z)^{n-1}}\left[P_{2}\left(\frac{1}{z_{1}}, \frac{z_{1}}{z_{2}}\right)-z_{1} P_{1}\left(\frac{1}{z_{2}}, \frac{z_{1}}{z_{2}}\right),-z_{2} P_{1}\left(\frac{y_{1}}{y_{3}}, \frac{y_{2}}{y_{3}}\right)\right]
$$

Pela mesma razão, (1.15) e (1.16) estarão multiplicados por $(-1)^{n-1}$ na expressão do campo $\bar{X}$ em $V_{2}$ e $V_{3}$, respectivamente. 
Desde que a multiplicação por um fator não nulo não altera o retrato de fase a menos pela inversão do sentido de todas as órbitas, no que segue, omitiremos o fator $\Delta(z)$ em $\bar{X}$. Então, a expressão do campo compactificado $\bar{X}$ torna-se um campo vetorial polinomial em cada carta local.

É importante observar que quando $n$ é ímpar, $\left.\bar{X}\right|_{V_{1}}=\left.\bar{X}\right|_{U_{1}}$. Logo, as trajetórias e pontos críticos de $X$ em $U_{1}$ e $V_{1}$ são os mesmos antes e depois da multiplicação pelo fator $y_{3}^{n-1}$. Quando $n$ é par, $\left.\bar{X}\right|_{V_{1}}=-\left.\bar{X}\right|_{U_{1}}$. As trajetórias e pontos críticos de $X$ em $U_{1}$ e $V_{1}$ coincidem antes e depois da multiplicação pelo fator $y_{3}^{n-1}$, mas ocorre uma mudança no sentido das órbitas de $X$ em $V_{1}, V_{2}$ e $V_{3}$ com relação às de $X$ em $U_{1}, U_{2}$ e $U_{3}$.

Nosso propósito, agora, é provar que o equador $\mathbb{S}^{1}$ é um conjunto invariante. De fato, para qualquer $y \in \mathbb{S}^{1}$, temos $y_{3}=0$, e desde que $z_{2}=\frac{y_{3}}{y_{i}}, i=1,2$, segue que $z_{2}=0$. Agora, o vetor tangente a $y \in \mathbb{S}^{1}$ é dado por (1.14) ou (1.15) ou uma expressão similar, e vemos que $z_{2}=0$ implica que a segunda componente do vetor tangente é sempre zero. Logo, $\mathbb{S}^{1}$ é invariante. Observe, que nesta análise, não precisamos nos preocupar com $y \in U_{3}$ ou $y \in V_{3}$, pois para esses casos $y_{3}>0$.

Como consequência do Teorema 1.4.2, temos que, dentre os pontos singulares hiperbólicos, somente pontos do tipo nós e selas podem ser encontradas no infinito. Dentre os pontos nilpotentes não podemos encontrar pontos do tipo cúspide, foco e centro. Isso acontece porque os pontos singulares do tipo cúspide, centro e foco não possuem curvas invariantes. Todos os pontos semi-hiperbólicos podem ser encontrados no infinito. Os pontos do tipo nó, sela e sela-nó têm, pelo menos, uma curva invariante.

Note que para o estudo global do campo $X$ (incluindo seu comportamento próximo ao infinito), é suficiente estudá-lo em $H_{+} \cup \mathbb{S}^{1}$.

Chamamos de ponto singular finito (respectivamente infinito) de $X$ ou $\bar{X}$ ao ponto singular de $\bar{X}$ que se encontra em $\mathbb{S}^{2} \backslash \mathbb{S}^{1}$ (respectivamente $\mathbb{S}^{1}$ ). Observe que, se $y \in \mathbb{S}^{1}$ é um ponto singular infinito, então $-y$, seu oposto com relação à origem, é também um ponto singular. Como o comportamento local próximo de $-y$ é o comportamento local próximo de $y$ multiplicado por $(-1)^{n-1}$, segue que a orientação das órbitas de $X$ muda quando o grau é par. Por exemplo, se $n$ é par e $y \in \mathbb{S}^{1}$ é um nó estável de $\bar{X}$, então $-y$ é um nó instável. Do fato que pontos singulares infinitos aparecem em pares de pontos 
opostos diametralmente, é suficiente estudar apenas a metade deles, e usando o grau do campo vetorial pode-se determinar a outra metade. A fim de desenhá-lo, por propósitos práticos, como um disco no plano, podemos projetar os pontos do hemisfério norte fechado no disco $\left\{\left(y_{1}, y_{2}, y_{3}\right) \in \mathbb{R}^{3}: y_{1}^{2}+y_{2}^{2} \leq 1, y_{3}=0\right\}$. Isto é feito pela projeção ortogonal de cada ponto da esfera sobre o disco.

Definição 1.4.3. A projeção do hemisfério norte fechado da esfera $\left(H_{+} \cup \mathbb{S}^{1}\right)$ em $y_{3}=0$ é chamada disco de Poincaré.

Vejamos a seguir um exemplo de como obter o retrato de fase global de um campo vetorial no disco de Poincaré.

Exemplo 1.4.4. Considere o campo vetorial linear $X=(x,-y)$. Este campo tem um único ponto singular finito, a origem, que é do tipo sela.

A expressão de $\rho(X)$ na carta $U_{1}$ é:

$$
\left\{\begin{array}{l}
\dot{z}_{1}=-2 z_{1} \\
\dot{z}_{2}=-z_{2}
\end{array}\right.
$$

Portanto, a origem é o único ponto singular em $U_{1}$, e é do tipo nó estável. Como o grau de $X$ é ímpar, a origem de $V_{1}$ é também um ponto singular do tipo nó estável.

A expressão de $\rho(X)$ na carta $U_{2}$ é:

$$
\left\{\begin{array}{l}
\dot{z}_{1}=2 z_{1} \\
\dot{z}_{2}=z_{2}
\end{array}\right.
$$

A origem de $U_{2}$ é ponto singular do tipo nó instável. Portanto, a origem de $V_{2}$ também é ponto singular do tipo nó instável.

Observe na Figura 1.18 o retrato de fase global do campo $X$ no disco de Poincaré.

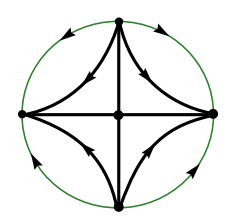

Figura 1.18: Retrato de fase global no disco de Poincaré. 



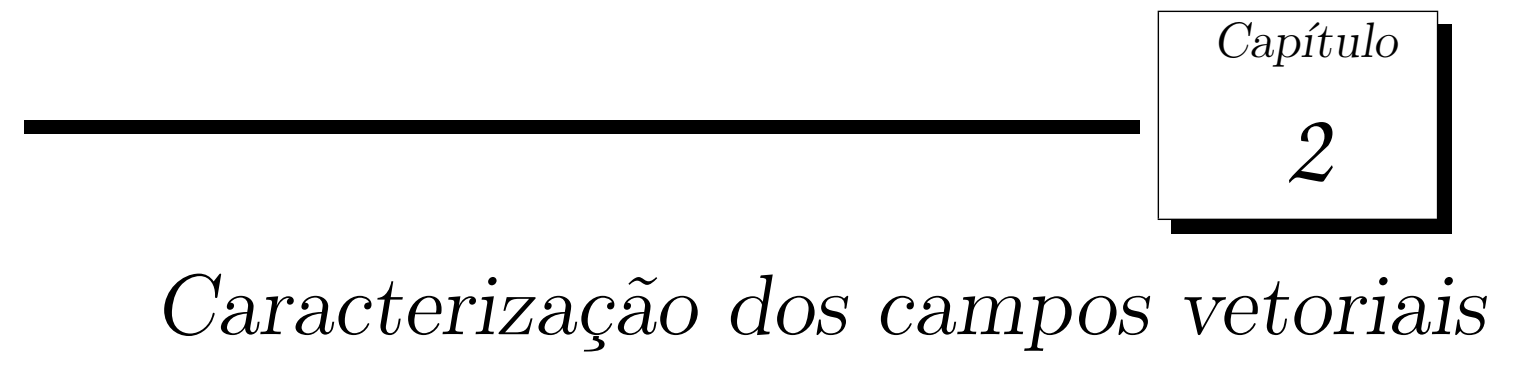

O objetivo desse capítulo é, baseado em [5], descrever em detalhes a metodologia utilizada para se obter a classificação global de todos os retratos de fase dos campos vetoriais quadráticos que possuem integral primeira racional de grau 2, no disco de Poincaré. Observemos que se $H$ é integral primeira racional de grau 2 do campo $X$, então, $H$ tem a forma:

$$
H=\frac{H_{1}}{H_{2}}=\frac{a_{00}+a_{10} x+a_{01} y+a_{20} x^{2}+a_{11} x y+a_{02} y^{2}}{b_{00}+b_{10} x+b_{01} y+b_{20} x^{2}+b_{11} x y+b_{02} y^{2}}
$$

onde $a_{20}^{2}+a_{11}^{2}+a_{02}^{2}+b_{20}^{2}+b_{11}^{2}+b_{02}^{2} \neq 0$ e com o numerador e denominador diferentes de uma constante, isto é, não aceitaremos que $H$ ou $\frac{1}{H}$ seja um polinômio.

Como observamos no Capítulo 1, os campos vetoriais quadráticos com integral primeira racional de grau 2 têm todas as suas órbitas contidas em cônicas, portanto, suas órbitas são curvas muito simples, mas isto não impede que seus retratos de fase possam apresentar uma rica variedade de dinâmicas. O resultado abaixo justifica tal afirmação.

Teorema 2.0.5. O retrato de fase de um campo vetorial polinomial quadrático planar com integral primeira racional de grau 2 é topologicamente equivalente a um dos 18 retratos de fase descritos na Figura 2.1, exceto pela reversão do sentido de todas as órbitas.

No que segue, trabalhamos na prova do Teorema 2.0.5. Nosso estudo se divide em duas etapas. Na primeira etapa, estudamos dois resultados auxiliares: o Lema 2.0.6, que estabelece condições sobre os coeficientes de $H$ (em (2.1)) para que esta seja integral primeira de um campo quadrático no plano e a Proposição 2.0.7 que reduz o estudo desejado em 9 casos. 


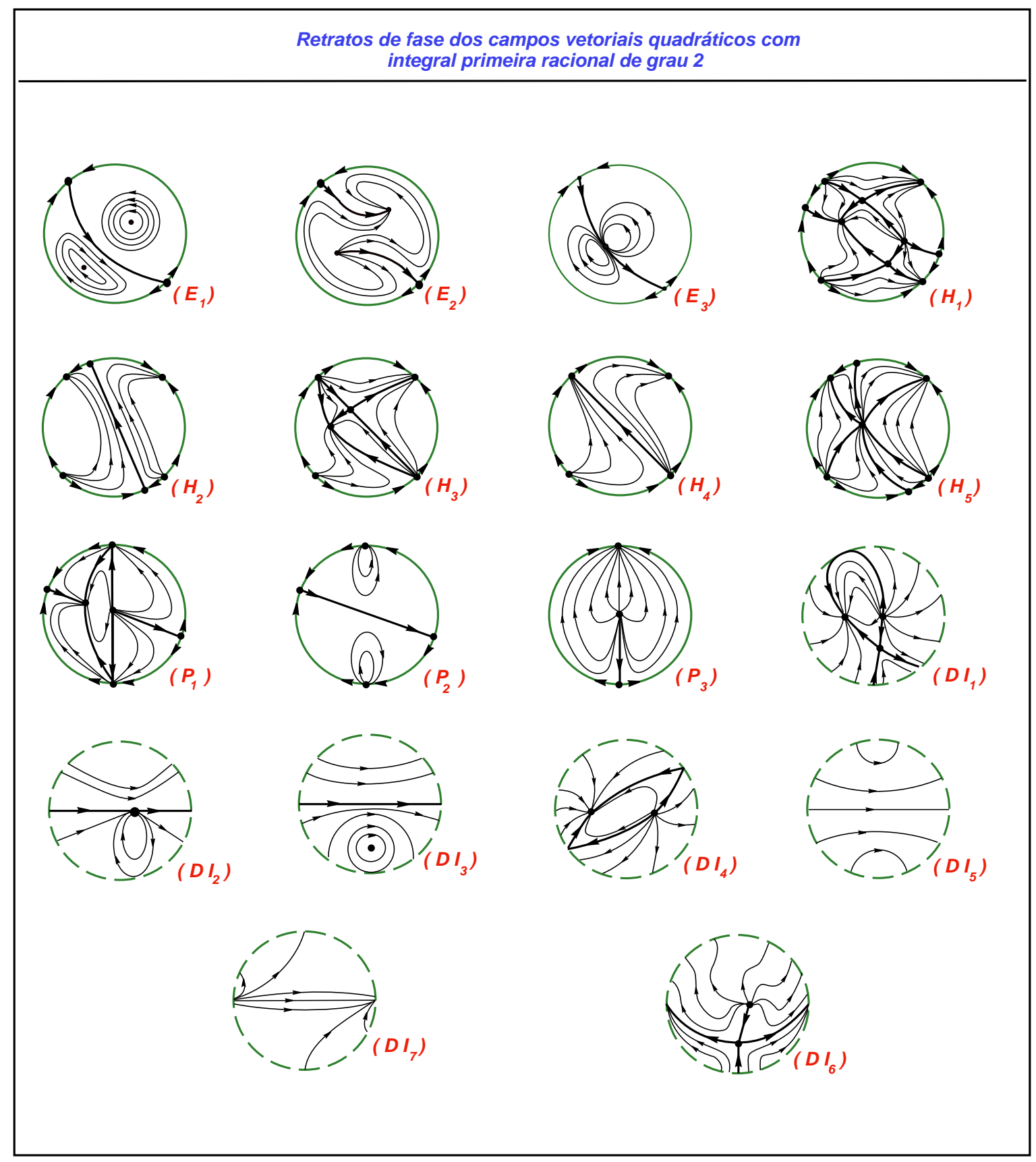

Figura 2.1: Retratos de fase globais dos campos quadráticos com integral primeira racional de grau 2 no disco de Poincaré. 
Lema 2.0.6. Para estudar todos os campos vetoriais quadráticos com uma função racional de grau 2 como integral primeira é suficiente estudar os campos vetoriais polinomiais quadráticos com uma integral primeira (2.1) tal que seus coeficientes satisfaçam um dos seguintes conjuntos de condições:

$$
\begin{aligned}
& a_{20} b_{11}-a_{11} b_{20}=0, \quad a_{20} b_{02}-a_{02} b_{20}=0, \quad a_{11} b_{02}-a_{02} b_{11}=0, \\
& \left(a_{01} b_{20}-a_{20} b_{01}+a_{11} b_{10}-a_{10} b_{11}\right)^{2}+\left(a_{02} b_{10}-a_{10} b_{02}\right)^{2}+ \\
& \left(a_{02} b_{01}-a_{01} b_{02}\right)^{2}+\left(a_{20} b_{01}-a_{01} b_{20}\right)^{2}+\left(a_{20} b_{01}-a_{01} b_{20}\right)^{2}+ \\
& \left(a_{10} b_{02}-a_{02} b_{10}+a_{11} b_{01}-a_{01} b_{11}\right)^{2} \neq 0, \\
& a_{00} b_{01}-a_{01} b_{00}=0, \quad a_{10} b_{01}-a_{11} b_{00}-a_{01} b_{10}+a_{00} b_{11}=0, \\
& a_{20} b_{01}-a_{11} b_{10}+a_{10} b_{11}-a_{01} b_{20}=0, \quad a_{20} b_{11}-a_{11} b_{20}=0, \\
& a_{10} b_{00}-a_{00} b_{10}=0, \quad a_{20} b_{00}-a_{00} b_{20}=0, \quad a_{20} b_{10}-a_{10} b_{20}=0, \\
& b_{11}^{2}+b_{20}^{2} \neq 0
\end{aligned}
$$

Além disso, os campos vetoriais com integral primeira (2.1) satisfazendo (2.3) são da forma:

$$
\left(2 b_{00}+2 b_{10} x+b_{01} y+2 b_{20} x^{2}+b_{11} x y, y\left(b_{10}+2 b_{20} x+b_{11} y\right)\right)
$$

e a integral primeira (2.1) pode ser simplificada em:

$$
H=\frac{y^{2}}{b_{00}+b_{10} x+b_{01} y+b_{20} x^{2}+b_{11} x y+b_{02} y^{2}} .
$$

\section{Demonstração:}

Suponha que $X=(P, Q)$ é um campo vetorial polinomial com integral primeira (2.1). Logo, $P$ e $Q$ satisfazem as seguintes condições:

$$
P=-\frac{\partial H}{\partial y}=\frac{-H_{1 y} H_{2}+H_{1} H_{2 y}}{H_{2}^{2}} \text { e } Q=\frac{\partial H}{\partial x}=\frac{H_{1 x} H_{1}-H_{1} H_{2 x}}{H_{2}^{2}},
$$

onde $H_{i y}$ e $H_{i x}$ indicam as derivadas parciais de $H_{i}$ com respeito a $x$ e $y$ com $i=1,2$, respectivamente.

Como a multiplicação por um fator positivo não altera o retrato de fase de um campo, consideramos (2.6) multiplicada por $H_{2}^{2}$ e:

$$
P=-H_{1 y} H_{2}+H_{1} H_{2 y} \text { e } Q=H_{1 x} H_{1}-H_{1} H_{2 x}
$$


isto é,

$$
\begin{aligned}
& P=-\left[\left(a_{01} b_{00}-a_{00} b_{01}\right)+2\left(a_{02} b_{00}-a_{00} b_{02}\right) y+\left(a_{11} b_{00}-a_{10} b_{01}+a_{01} b_{10}-a_{00} b_{11}\right) x\right. \\
& +\left(a_{01} b_{20}-a_{20} b_{01}+a_{11} b_{10}-a_{10} b_{11}\right) x^{2}+2\left(a_{02} b_{10}-a_{10} b_{02}\right) x y+\left(a_{02} b_{01}-a_{01} b_{02}\right) y^{2} \\
& \left.+2\left(a_{02} b_{20}-a_{20} b_{02}\right) x^{2} y+\left(a_{02} b_{11}-a_{11} b_{02}\right) x y^{2}+\left(a_{11} b_{20}-a_{20} b_{11}\right) x^{3}\right]
\end{aligned}
$$

e

$$
\begin{aligned}
& Q=\left(a_{10} b_{00}-a_{00} b_{10}\right)+2\left(a_{20} b_{00}-a_{00} b_{20}\right) x+\left(a_{11} b_{00}-a_{01} b_{10}+a_{10} b_{01}-a_{00} b_{11}\right) y \\
& +\left(a_{20} b_{10}-a_{10} b_{20}\right) x^{2}+2\left(a_{20} b_{01}-a_{01} b_{20}\right) x y+\left(a_{10} b_{02}-a_{02} b_{10}+a_{11} b_{01}-a_{01} b_{11}\right) y^{2} \\
& +\left(a_{20} b_{11}-a_{11} b_{20}\right) x^{2} y+2\left(a_{20} b_{02}-a_{02} b_{20}\right) x y^{2}+\left(a_{11} b_{02}-a_{02} b_{11}\right) y^{3} .
\end{aligned}
$$

Sendo assim, existem duas possibilidades para o campo vetorial $(P, Q)$ ser quadrático: ou todos os coeficientes dos termos cúbicos de $P$ e $Q$ são nulos, ou $P$ e $Q$ possuem um fator comum de grau 1.

No primeiro caso, igualando a zero os coeficientes dos termos cúbicos de (2.7) e (2.8), obtemos:

$$
\begin{aligned}
& -2\left(a_{02} b_{20}-a_{20} b_{02}\right)=0 \\
& -\left(a_{02} b_{11}-a_{11} b_{02}\right)=0 \\
& -\left(a_{11} b_{20}-a_{20} b_{11}\right)=0
\end{aligned}
$$

Note que essas condições garantem que $(P, Q)$ tem grau menor ou igual a 2. Em outras palavras, essas condições não impedem que $(P, Q)$ seja linear. Como buscamos $X=(P, Q)$ de grau exatamente 2 , os coeficientes dos termos quadráticos de $P$ e $Q$ não podem se anular simultaneamente, para isso, estabelecemos a quarta condição abaixo:

$$
\begin{aligned}
& \left(a_{01} b_{20}-a_{20} b_{01}+a_{11} b_{10}-a_{10} b_{11}\right)^{2}+\left(a_{02} b_{10}-a_{10} b_{02}\right)^{2}+\left(a_{02} b_{01}-a_{01} b_{02}\right)^{2} \\
& \left(a_{20} b_{10}-a_{10} b_{20}\right)^{2}+\left(a_{20} b_{01}-a_{01} b_{20}\right)^{2}+\left(a_{10} b_{02}-a_{02} b_{10}+a_{11} b_{01}-a_{01} b_{11}\right)^{2} \neq 0 .
\end{aligned}
$$

Dessa maneira, obtemos as quatro condições de (2.2).

No segundo caso, podemos supor, sem perda de generalidade, que $y$ é um fator comum de $P$ e $Q$ (se necessário, fazemos uma mudança linear de variáveis). Sendo assim, o termo constante e os coeficientes de $x, x^{2}$ e $x^{3}$ de $P$ e $Q$ são nulos e obtemos as 7 condições abaixo: 


$$
\begin{gathered}
-\left(a_{01} b_{00}-a_{00} b_{01}\right)=0 \\
-\left(a_{11} b_{00}-a_{10} b_{01}+a_{01} b_{10}-a_{00} b_{11}\right)=0 \\
-\left(a_{01} b_{20}-a_{20} b_{01}+a_{11} b_{10}-a_{10} b_{11}\right)=0 \\
-\left(a_{11} b_{20}-a_{20} b_{11}\right)=0 \\
\left(a_{10} b_{00}-a_{00} b_{10}\right)=0 \\
2\left(a_{20} b_{00}-a_{00} b_{20}\right)=0 \\
\left(a_{20} b_{10}-a_{10} b_{20}\right)=0 .
\end{gathered}
$$

Resolvendo esse sistema de sete equações, obtemos cinco possíveis soluções. A seguir, estudamos cada uma delas.

Solução 1: $b_{10} \neq 0, a_{01}=\frac{a_{10} b_{01}}{b_{10}}, a_{11}=\frac{a_{10} b_{11}}{b_{10}}, a_{20}=\frac{a_{10} b_{20}}{b_{10}}$ e $a_{00}=\frac{a_{10} b_{00}}{b_{10}}$. Isso nos leva a

$$
\begin{aligned}
& H=\frac{a_{10} b_{00}+a_{10} b_{10} x+a_{10} b_{20} x^{2}+a_{10} b_{01} y+a_{10} b_{11} x y+a_{02} b_{10} y^{2}}{b_{10}\left(b_{00}+b_{10} x+b_{20} x^{2}+b_{01} y+b_{11} x y+b_{02} y^{2}\right)}, \\
& P=\frac{1}{b_{10}}\left(\left(a_{10} b_{02}-a_{02} b_{10}\right) y\left(2 b_{00}+2 b_{10} x+2 b_{20} x^{2}+b_{01} y+b_{11} x y\right)\right), \\
& Q=\frac{1}{b_{10}}\left(\left(a_{10} b_{02}-a_{02} b_{10}\right) y^{2}\left(b_{10}+2 b_{20} x+b_{11} y\right)\right) .
\end{aligned}
$$

Supondo $\left(a_{10} b_{02}-a_{02} b_{10}\right) \neq 0$ (caso contrário, $(P, Q)=0$ para todo $\left.(x, y) \in \mathbb{R}^{2}\right)$ dividimos $P$ e $Q$ pelo fator comum $\frac{1}{b_{10}}\left(a_{10} b_{02}-a_{02} b_{10}\right) y$ e

$$
\begin{aligned}
& \bar{P}=2 b_{00}+2 b_{10} x+2 b_{20} x^{2}+b_{01} y+b_{11} x y, \\
& \bar{Q}=y\left(b_{10}+2 b_{20} x+b_{11} y\right) .
\end{aligned}
$$

Além disso, como vimos no Capítulo 1

$$
\bar{H}=\frac{-a_{10}+H b_{10}}{-a_{10} b_{02}+a_{02} b_{10}}=\frac{y^{2}}{b_{00}+b_{10} x+b_{20} x^{2}+b_{01} y+b_{11} x y+b_{02} y^{2}}
$$

é integral primeira do sistema, se $-a_{10} b_{02}+a_{02} b_{10} \neq 0$.

Solução 2: $a_{00}=a_{20}=a_{10}=b_{00}=b_{20}=b_{10}=0$. Neste caso, 


$$
\begin{aligned}
& H=\frac{a_{01}+a_{11} x+a_{02} y}{b_{01}+b_{11} x+b_{02} y} \\
& P=-\left(a_{02} b_{01}-a_{01} b_{02}-a_{11} b_{02} x+a_{02} b_{11} x\right) y^{2}, \\
& Q=\left(a_{11} b_{01}-a_{01} b_{11}+a_{11} b_{02} y-a_{02} b_{11} y\right) y^{2} .
\end{aligned}
$$

Note que $H$ tem grau 1 e $\left(P / y^{2}, Q / y^{2}\right)$ é um campo linear. temos

Solução 3: $b_{00} \neq 0, a_{10}=b_{10}=0, a_{01}=\frac{a_{00} b_{01}}{b_{00}}, a_{11}=\frac{a_{00} b_{11}}{b_{00}}, a_{20}=\frac{a_{00} b_{20}}{b_{00}}$. Aqui

$$
\begin{aligned}
& H=\frac{a_{00} b_{00}+a_{00} b_{20} x^{2}+a_{00} b_{01} y+a_{00} b_{11} x y+a_{02} b_{00} y^{2}}{b_{00}\left(b_{00}+b_{20} x^{2}+b_{01} y+b_{11} x y+b_{02} y^{2}\right)}, \\
& P=\frac{-1}{b_{00}}\left(\left(a_{02} b_{00}-a_{00} b_{02}\right) y\left(2 b_{00}+2 b_{20} x^{2}+b_{01} y+b_{11} x y\right)\right), \\
& Q=\frac{-1}{b_{00}}\left(\left(a_{02} b_{00}-a_{00} b_{02}\right) y^{2}\left(2 b_{20} x+b_{11} y\right)\right) .
\end{aligned}
$$

Assim, supondo $\left(a_{02} b_{00}-a_{00} b_{02}\right) \neq 0$, dividindo $P$ e $Q$ por $\frac{-1}{b_{00}}\left(a_{02} b_{00}-a_{00} b_{02}\right) y$ temos:

$$
\begin{aligned}
& \bar{P}=2 b_{00}+2 b_{20} x^{2}+b_{01} y+b_{11} x y, \\
& \bar{Q}=y\left(2 b_{20} x+b_{11} y\right) .
\end{aligned}
$$

Além disso,

$$
\bar{H}=\frac{-a_{00}+H b_{00}}{a_{02} b_{00}-a_{00} b_{02}}=\frac{y^{2}}{b_{00}+b_{20} x^{2}+b_{01} y+b_{11} x y+b_{02} y^{2}}
$$

é integral primeira do campo.

Observe que esta solução corresponde à Solução 1 quando $b_{10}=0$.

Solução 4: $b_{20} \neq 0, b_{00}=a_{00}=a_{10}=b_{10}=0, a_{01}=\frac{a_{20} b_{01}}{b_{20}}, a_{11}=\frac{a_{20} b_{11}}{b_{20}}$. Neste caso, 


$$
\begin{aligned}
& H=\frac{a_{20} b_{20} x^{2}+a_{20} b_{01} y+a_{20} b_{11} x y+a_{02} b_{20} y^{2}}{b_{20}\left(b_{20} x^{2}+b_{01} y+b_{11} x y+b_{02} y^{2}\right)}, \\
& P=\frac{1}{b_{20}}\left(\left(a_{20} b_{02}-a_{02} b_{20}\right) y\left(2 b_{20} x^{2}+b_{01} y+b_{11} x y\right)\right), \\
& Q=\frac{1}{b_{20}}\left(\left(a_{20} b_{02}-a_{02} b_{20}\right) y^{2}\left(2 b_{20} x+b_{11} y\right)\right) .
\end{aligned}
$$

Novamente, temos a Solução 1 quando $b_{00}=b_{10}=0$.

Solução 5: $b_{02} \neq 0, b_{01}=b_{11}=b_{20}=b_{00}=b_{10}=0$. Nessas condições, temos

$$
\begin{gathered}
H=\frac{a_{00}+a_{10} x+a_{01} y+a_{20} x^{2}+a_{11} x y+a_{02} y^{2}}{b_{02} y^{2}}, \\
(P, Q)=\left(b_{02} y\left(2 a_{00}+2 a_{10} x+2 a_{20} x^{2}+a_{01} y+a_{11} x y\right), b_{02} y^{2}\left(a_{10}+2 a_{20} x+a_{11} y\right)\right) .
\end{gathered}
$$

Lembrando que, se $H$ é integral primeira de um campo vetorial, então, $\frac{1}{H}$ também é, concluímos que esta solução também corresponde à Solução 1 (basta trocarmos $a_{i j}$ por $b_{i j}$ com $i, j=0,1,2)$.

Finalmente, após a análise das 5 soluções de (2.3), concluímos que os campos vetoriais quadráticos com integral primeira (2.1) satisfazendo (2.3) são da forma

$$
\left(2 b_{00}+2 b_{10} x+b_{01} y+2 b_{20} x^{2}+b_{11} x y, y\left(b_{10}+2 b_{20} x+b_{11} y\right)\right)
$$

e a integral primeira $H$ em (2.1) pode ser simplificada em:

$$
H=\frac{y^{2}}{b_{00}+b_{10} x+b_{01} y+b_{20} x^{2}+b_{11} x y+b_{02} y^{2}} .
$$

Proposição 2.0.7. Uma integral primeira racional de grau 2 pode ser reduzida, depois de uma mudança afim de variáveis, a uma das seguintes nove formas:

$$
\frac{\left(x^{2}+y^{2}-1\right)}{D(x, y)}(E) \quad \frac{\left(x^{2}+y^{2}\right)}{D(x, y)}(p) \quad \frac{\left(x^{2}-1\right)}{D(x, y)}(P L)
$$




$$
\begin{array}{lll}
\frac{\left(x^{2}+y^{2}+1\right)}{D(x, y)}(C E) & \frac{x y}{D(x, y)}(L V) & \frac{\left(x^{2}+1\right)}{D(x, y)}(C L) \\
\frac{\left(x^{2}-y^{2}-1\right)}{D(x, y)}(H) & \frac{\left(x^{2}-y\right)}{D(x, y)}(P) & \frac{x^{2}}{D(x, y)}(D L)
\end{array}
$$

onde a cônica do numerador é uma elipse, uma elipse complexa, uma hiperbóle, duas retas complexas interceptando-se em um ponto real, duas retas reais com intersecção, uma parábola, duas retas reais paralelas, duas retas complexas paralelas, uma reta real dupla, respectivamente. Aqui $D(x, y)$ denota um polinômio arbitrário de grau 2.

Esta proposição é uma consequência direta da classificação das cônicas.

A segunda etapa da prova do Teorema 2.0.5 consiste em, primeiramente, para cada uma das nove formas de $H$ na Proposição 2.0.7, analisarmos o campo vetorial polinomial quadrático, satisfazendo as condições (2.2). Obtemos quatorze dos vinte e um retratos de fase no disco de Poincaré, apresentados na Figura 2.1. Dessa forma, analisando os campos vetoriais quadráticos da forma (2.4), obtemos os outros sete retratos de fase no disco de Poincaré apresentados na Figura 2.1.

A técnica utilizada para obtenção dos retratos de fase divide-se em três etapas:

- Etapa 1: estudo dos pontos singulares infinitos. Nessa etapa encontramos os pontos singulares infinitos por meio da Compactificação de Poincaré. Em seguida, calculamos a matriz Jacobiana do campo compactificado em cada singularidade. Classificamos as singularidades em hiperbólica, semi-hiperbólica, nilpotente ou linearmente nula. Utilizando a classificação dos pontos elementares descrita no Capítulo 1, obtemos o retrato de fase numa vizinhança de cada ponto singular infinito.

- Etapa 2: estudo dos pontos singulares finitos. Classificamos as singularidades locais (análise análoga à realizada para os pontos singulares infinitos).

- Etapa 3: desenho do retrato de fase no disco de Poincaré. Reunindo as informações obtidas nas duas etapas anteriores, usando a continuidade das órbitas e a integral primeira do sistema, construímos o retrato de fase global do campo vetorial quadrático no disco de Poincaré.

Abaixo, estudamos os sistemas diferenciais, cujas integrais primeiras têm uma das nove formas normais apresentadas na Proposição 2.0.7 com o objetivo de conhecer os retratos de fase globais de tais sistemas. 


\subsection{Elipse}

Se a integral primeira racional é da forma $\frac{\left(x^{2}+y^{2}-1\right)}{D(x, y)}$ segue de $(2.1)$ que $a_{00}=-1$, $a_{10}=a_{01}=a_{11}=0$ e $a_{20}=a_{02}=1$. Além disso, da primeira e segunda equação de $(2.2)$ seguem, respectivamente, que $b_{11}=0$ e $b_{20}=b_{02}$. Então o sistema cuja integral primeira tem a forma acima é:

$$
\begin{aligned}
& \dot{x}=P(x, y)=-b_{01}-2\left(b_{00}+b_{20}\right) y+b_{01} x^{2}-2 b_{10} x y-b_{01} y^{2} \\
& \dot{y}=Q(x, y)=b_{10}+2\left(b_{00}+b_{20}\right) x+b_{10} x^{2}+2 b_{01} x y-b_{10} y^{2} .
\end{aligned}
$$

Definamos $r^{2}=b_{01}^{2}+b_{10}^{2}, p=b_{00}+b_{20}$ e $q=p^{2}-r^{2}$. Observamos que se $r^{2}=0$ então $b_{01}=b_{10}=0$ e, neste caso, $(P, Q)$ é campo linear. Logo, podemos assumir $r^{2}>0$.

\section{Análise dos pontos singulares infinitos.}

Após a compactificação de Poincaré na carta $U_{1}$, o campo vetorial (2.11) é da forma:

$$
\begin{aligned}
& \dot{z}_{1}=b_{10}+b_{01} z_{1}+2 p z_{2}+b_{10} z_{1}^{2}+b_{10} z_{2}^{2}+b_{01} z_{1}^{3}+2 p z_{1}^{2} z_{2}+b_{01} z_{1} z_{2}^{2} \\
& \dot{z_{2}}=-b_{01} z_{2}+2 b_{10} z_{1} z_{2}+b_{01} z_{1}^{2} z_{2}+2 p z_{1} z_{2}^{2}+b_{01} z_{2}^{3}
\end{aligned}
$$

Se $b_{01} \neq 0$, o único ponto singular em $U_{1}$ é $\left(-\frac{b_{10}}{b_{01}}, 0\right)$, no qual o sistema tem matriz Jacobiana

$$
J X\left(-\frac{b_{10}}{b_{01}}, 0\right)=\left[\begin{array}{cc}
\frac{r^{2}}{b_{01}} & \frac{2 r^{2} p}{b_{01}^{2}} \\
0 & -\frac{r^{2}}{b_{01}}
\end{array}\right]
$$

e, portanto, é uma singularidade do tipo sela.

Se $b_{01}=0$, não existem pontos singulares na carta $U_{1}$. Para completar o estudo do campo no infinito, como já observamos no Capítulo 1.4, basta estudar a origem da carta $U_{2}$.

O campo vetorial na carta $U_{2}$ é da forma:

$$
\begin{aligned}
& \dot{z}_{1}=-b_{01}-b_{10} z_{1}-2 p z_{2}-b_{01} z_{1}^{2}-b_{01} z_{2}^{2}-b_{10} z_{1}^{3}-2 p z_{1}^{2} z_{2}-b_{10} z_{1} z_{2}^{2} \\
& \dot{z}_{2}=b_{10} z_{2}-2 b_{01} z_{1} z_{2}-b_{10} z_{1}^{2} z_{2}-2 p z_{1} z_{2}^{2}-b_{10} z_{2}^{3}
\end{aligned}
$$


Nesta carta, a origem é um ponto singular apenas se $b_{01}=0$, e tem matriz Jacobiana dada por:

$$
J X(0,0)=\left[\begin{array}{cc}
-b_{10} & -2 p \\
0 & b_{10}
\end{array}\right]
$$

Logo, a origem da carta $U_{2}$ é uma singularidade do tipo sela se $b_{01}=0$. Caso contrário, a origem não é ponto singular do sistema.

Encontrados os pontos singulares infinitos em $U_{1}$ e $U_{2}$, por simetria, obtemos os pontos correspondentes em $V_{1}$ e $V_{2}$. Como já observamos, os pontos singulares em $V_{1}$ e $V_{2}$ têm a orientação de suas órbitas oposta à de seus correspondentes pontos em $U_{1}$ e $U_{2}$, pois o campo vetorial (2.11) tem grau par.

Na Figura 2.2, apresentamos um esboço de como desenhar os pontos singulares infinitos no disco de Poincaré. Observe que, independente de $b_{01}=0, b_{01} \neq 0,-\frac{b_{10}}{b_{01}}>0 \mathrm{e}-\frac{b_{10}}{b_{01}}<0$, a configuração dos pontos singulares infinitos será a mesma, a menos de uma rotação do disco.
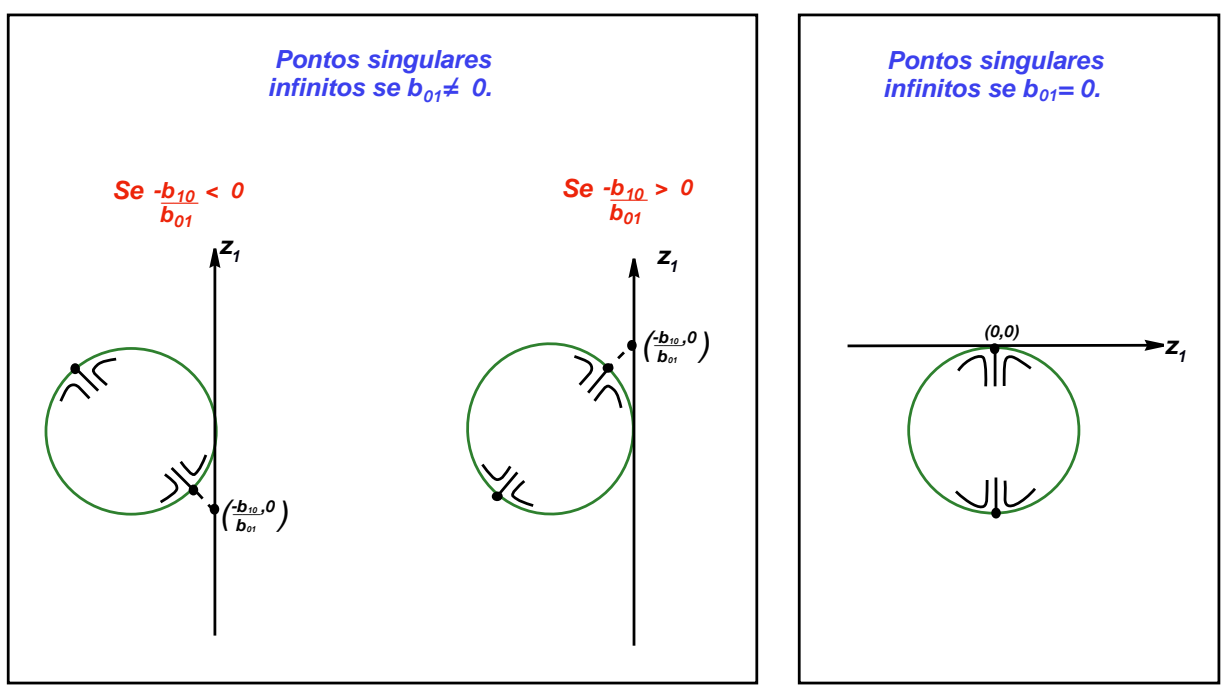

Figura 2.2: Pontos singulares infinitos para o caso $(E)$.

\section{Análise dos pontos singulares finitos.}

O sistema $P=Q=0$ em (2.11) tem quatro soluções. São elas: 


$$
\begin{gathered}
P_{1}=\frac{-p-\sqrt{q}}{r^{2}}\left(b_{10}, b_{01}\right), \\
P_{2}=\frac{-p+\sqrt{q}}{r^{2}}\left(b_{10}, b_{01}\right), \\
P_{3}=\frac{1}{r^{2}}\left(-b_{10} p-b_{01} \sqrt{-q},-b_{01} p+b_{10} \sqrt{-q}\right), \\
P_{4}=\frac{1}{r^{2}}\left(-b_{10} p+b_{01} \sqrt{-q},-b_{01} p-b_{10} \sqrt{-q}\right) .
\end{gathered}
$$

Distinguimos os três casos:

i) $q>0$. Nesse caso, $P_{1}$ e $P_{2}$ são as únicas singularidades do sistema diferencial (2.11) e

$$
J X\left(P_{1}\right)=\left[\begin{array}{cc}
0 & 2 \sqrt{q} \\
-2 \sqrt{q} & 0
\end{array}\right], \quad J X\left(P_{2}\right)=\left[\begin{array}{cc}
0 & -2 \sqrt{q} \\
2 \sqrt{q} & 0
\end{array}\right],
$$

logo, $P_{1}$ e $P_{2}$ são centros ou focos fracos. Da existência da integral primeira racional de grau 2 segue que todas as órbitas do sistema estão contidas em cônicas e, como cônicas não podem conter espirais, concluímos que ambos os pontos singulares são centros.

Reunindo as informações obtidas para os pontos singulares infinitos podemos fazer um esboço do retrato de fase do campo (2.11) para este caso; veja Figura 2.3. Note, na Figura 2.3 que um retrato de fase é obtido do outro por uma inversão da orientação de todas as órbitas.

ii) $q<0 . P_{3}, P_{4}$ são as únicas singularidades do sistema diferencial (2.11). Como

$$
J X\left(P_{3}\right)=\left[\begin{array}{cc}
-2 \sqrt{-q} & 0 \\
0 & -2 \sqrt{-q}
\end{array}\right], \quad J X\left(P_{4}\right)=\left[\begin{array}{cc}
2 \sqrt{-q} & 0 \\
0 & 2 \sqrt{-q}
\end{array}\right]
$$

segue que, $P_{3}$ é um ponto singular do tipo nó estável e $P_{4}$ é do tipo nó instável. Lembrando que no infinito temos dois pontos singulares do tipo sela, decorre que o campo vetorial quadrático tem somente duas separatrizes vindas das duas selas no infinito. É conhecido que se existe uma curva invariante unindo duas selas no infinito, então, essa curva é uma reta (veja [21]).

Um esboço do retrato de fase do campo (2.11) para o caso $q<0$ é dado pela Figura 2.4 . 
Caso (1): $q>0$

Pontos singulares

Retrato de fase no infinitos e finitos. disco de Poincaré.
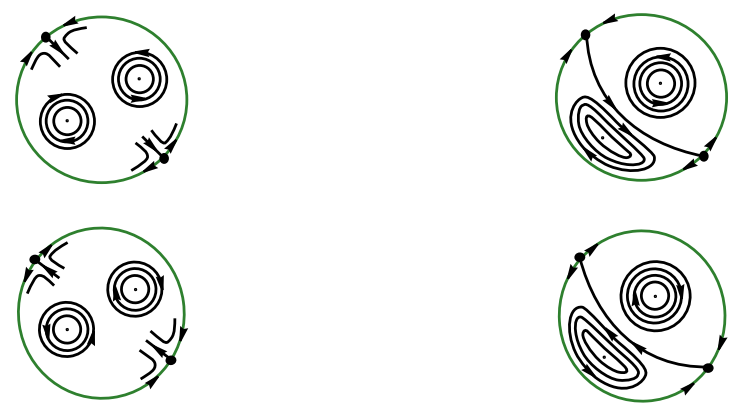

Figura 2.3: Retratos de fase no disco de Poincaré para o caso $(E)$ com $q>0$.

Note, na Figura 2.4, que um retrato de fase é obtido do outro por uma inversão da orientação de todas as órbitas.

Caso (2): $q<0$

Pontos singulares

infinitos e finitos.

Retrato de fase no
disco de Poincaré.
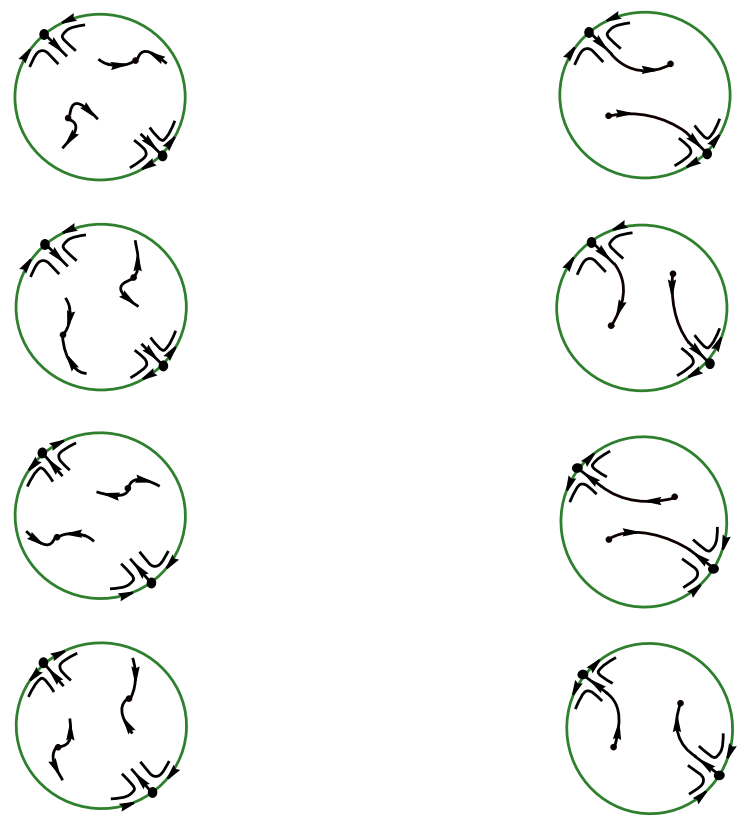

Figura 2.4: Retratos de fase no disco de Poincaré para o caso $(E)$ com $q<0$. 
iii) $q=0$. Existe um único ponto singular de multiplicidade quatro, que é $-\frac{1}{p}\left(b_{10}, b_{01}\right)$. Para este ponto, temos

$$
J X\left(-\frac{1}{p}\left(b_{10}, b_{01}\right)\right)=\left[\begin{array}{ll}
0 & 0 \\
0 & 0
\end{array}\right]
$$

e então $-\frac{1}{p}\left(b_{10}, b_{01}\right)$ é um ponto singular linearmente nulo.

Para estudar seu tipo topológico, devemos fazer, primeiramente, uma translação da singularidade para a origem. Aplicamos, então, a seguinte mudança de coordenadas

$$
\left\{\begin{array} { l } 
{ X = x + \frac { b _ { 1 0 } } { p } } \\
{ Y = y + \frac { b _ { 0 1 } } { p } }
\end{array} \Rightarrow \left\{\begin{array} { l } 
{ \dot { X } = \dot { x } } \\
{ \dot { Y } = \dot { y } }
\end{array} \Rightarrow \left\{\begin{array}{l}
\dot{X}=P\left(X-\frac{b_{10}}{p}, Y-\frac{b_{01}}{p}\right) \\
\dot{Y}=Q\left(X-\frac{b_{10}}{p}, Y-\frac{b_{01}}{p}\right)
\end{array}\right.\right.\right.
$$

e desta forma, obtemos:

$$
(P, Q)=\left(b_{01}\left(X^{2}-Y^{2}\right)-2 b_{10} X Y, b_{10}\left(X^{2}-Y^{2}\right)+2 b_{01} X Y\right)
$$

que é campo homogêneo. Portanto, seu retrato de fase é um dos seis retratos descritos na Figura 1.9.

Agora, note que dada $f(X, Y)=b_{10} X+b_{01} Y$, temos que

$$
\begin{aligned}
P \frac{\partial f}{\partial X}+Q \frac{\partial f}{\partial Y} & =2 b_{01} b_{10} X^{2}-2 b_{01} b_{10} Y^{2}-2 b_{10}^{2} X Y+2 b_{01}^{2} X Y \\
& =\left(2 b_{01} X-2 b_{10} Y\right)\left(b_{10} X+b_{01} Y\right)=k f
\end{aligned},
$$

onde $k(X, Y)=\left(2 b_{01} X-2 b_{10} Y\right)$. Portanto, $f(X, Y)=0$ é a reta invariante passando pela origem. Juntando a isto o fato que este campo vetorial tem duas selas no infinito, segue que o retrato de fase deste campo é topologicamente equivalente ao da Figura 1.9 a). Então, a origem e, consequentemente, o ponto singular $-\frac{1}{p}\left(b_{10}, b_{01}\right)$ é formado pela união de dois setores elípticos. Na Figura 2.5, apresentamos um esboço do retrato de fase do campo (2.11) no disco de Poincaré para este caso. 


\section{Caso (3): $q=0$}
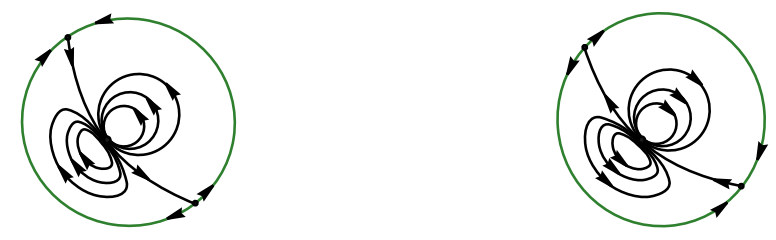

Figura 2.5: Retratos de fase no disco de Poincaré para o caso $(E)$.

Novamente, obtemos dois retratos de fase, onde um é obtido do outro por uma inversão da orientação de todas as órbitas.

Nas Figuras 2.3, 2.4 e 2.5, assumimos $-\frac{b_{10}}{b_{01}}<0$, para o caso contrário, obtemos retratos de fase já encontrados, a menos de uma rotação do disco.

Resumidamente, temos a seguinte proposição:

Proposição 2.1.1. O retrato de fase de um campo vetorial polinomial quadrático planar com uma integral primeira racional de grau 2 da forma (E) dada na Proposição (2.0.7) é topologicamente equivalente aos seguintes retratos de fase da Figura 2.1:

(i) $\left(E_{1}\right)$ se $q>0$;

(ii) $\left(E_{2}\right)$ se $q<0$;

(iii) $\left(E_{3}\right)$ se $q=0$.

\subsection{Elipse Complexa}

Se a integral primeira racional é da forma $\frac{\left(x^{2}+y^{2}+1\right)}{D(x, y)}$, de $(2.1)$ segue que $a_{00}=a_{20}=$ $a_{02}=1, a_{10}=a_{01}=a_{11}=0$. Além disso, da primeira e segunda equação de (2.2) seguem, respectivamente, que $b_{11}=0$ e $b_{20}=b_{02}$. Temos, então, o sistema:

$$
\begin{aligned}
& \dot{x}=P(x, y)=b_{01}-2\left(b_{00}-b_{20}\right) y+b_{01} x^{2}-2 b_{10} x y-b_{01} y^{2} \\
& \dot{y}=Q(x, y)=-b_{10}+2\left(b_{00}-b_{20}\right) x+b_{10} x^{2}+2 b_{01} x y-b_{10} y^{2}
\end{aligned}
$$


Definamos $r^{2}=b_{01}^{2}+b_{10}^{2}, p=b_{00}-b_{20}$ e $q=p^{2}+r^{2}$. Como no caso anterior, podemos assumir $r^{2}>0$, consequentemente $q>0$.

\section{Análise dos pontos singulares infinitos.}

Na carta $U_{1}$, o campo vetorial (2.12) é da forma:

$$
\begin{aligned}
& \dot{z}_{1}=b_{10}+b_{01} z_{1}+2 p z_{2}+b_{10} z_{1}^{2}-b_{10} z_{2}^{2}+b_{01} z_{1}^{3}+2 p z_{1}^{2} z_{2}-b_{01} z_{1} z_{2}^{2} \\
& \dot{z}_{2}=b_{01} z_{2}+2 b_{10} z_{1} z_{2}-b_{01} z_{1}^{2} z_{2}-2 p z_{1} z_{2}^{2}+b_{01} z_{2}^{3} .
\end{aligned}
$$

Se $b_{01} \neq 0$, o único ponto singular infinito em $U_{1}$ é $\left(-\frac{b_{10}}{b_{01}}, 0\right)$, o qual tem matriz Jacobiana

$$
J X\left(-\frac{b_{10}}{b_{01}}, 0\right)=\left[\begin{array}{cc}
\frac{r^{2}}{b_{01}} & \frac{2 r^{2} p}{b_{01}^{2}} \\
0 & -\frac{r^{2}}{b_{01}}
\end{array}\right]
$$

e, portanto, é uma singularidade do tipo sela.

Se $b_{01}=0$, não existem pontos singulares na carta $U_{1}$.

O campo vetorial na carta $U_{2}$ é da forma:

$$
\begin{aligned}
& \dot{z}_{1}=-b_{01}-b_{10} z_{1}-2 p z_{2}-b_{01} z_{1}^{2}-b_{01} z_{2}^{2}-b_{10} z_{1}^{3}-2 p z_{1}^{2} z_{2}+b_{10} z_{1} z_{2}^{2} \\
& \dot{z}_{2}=b_{10} z_{2}-2 b_{01} z_{1} z_{2}-b_{10} z_{1}^{2} z_{2}-2 p z_{1} z_{2}^{2}+b_{10} z_{2}^{3}
\end{aligned}
$$

A origem é um ponto singular se $b_{01}=0$, e tem matriz Jacobiana dada por:

$$
J X(0,0)=\left[\begin{array}{cc}
-b_{10} & -2 p \\
0 & b_{10}
\end{array}\right]
$$

Logo, a origem da carta $U_{2}$ é uma singularidade do tipo sela. Se $b_{01} \neq 0,(0,0)$ é ponto regular.

Encontrados os pontos singulares infinitos em $U_{1}$ e $U_{2}$, por simetria obtemos os pontos correspondentes em $V_{1}$ e $V_{2}$. Observe que o comportamento no infinito é idêntico ao caso elíptico (E). Um esboço dos pontos singulares infinitos no disco de Poincaré é dado na Figura 2.2. 
Análise dos pontos singulares finitos.

O sistema $P=Q=0$ em (2.12) tem quatro soluções. São elas:

$$
\begin{gathered}
P_{1}=\frac{-p-\sqrt{q}}{r^{2}}\left(b_{10}, b_{01}\right), \\
P_{2}=\frac{-p+\sqrt{q}}{r^{2}}\left(b_{10}, b_{01}\right), \\
P_{3}=\frac{1}{r^{2}}\left(-b_{10} p+b_{01} \sqrt{-q},-b_{01} p-b_{10} \sqrt{-q}\right),
\end{gathered}
$$

$\mathrm{e}$

$$
P_{4}=\frac{1}{r^{2}}\left(-b_{10} p-b_{01} \sqrt{-q},-b_{01} p+b_{10} \sqrt{-q}\right) .
$$

Como $q>0, P_{1}$ e $P_{2}$ são as singularidades do sistema diferencial (2.12) e como

$$
J X\left(x_{1}, y_{1}\right)=\left[\begin{array}{cc}
0 & 2 \sqrt{q} \\
-2 \sqrt{q} & 0
\end{array}\right], \quad J X\left(x_{2}, y_{2}\right)=\left[\begin{array}{cc}
0 & -2 \sqrt{q} \\
2 \sqrt{q} & 0
\end{array}\right],
$$

$P_{1}$ e $P_{2}$ são centros ou foco fracos. Como no caso elipse, da existência da integral primeira racional de grau 2 concluímos que os pontos singulares são centros.

Observe que o comportamento dos pontos singulares infinitos e finitos é análogo ao do caso elíptico (E) para $q>0$, e o retrato de fase do sistema coincide com o da Figura 2.3.

Resumidamente, temos a seguinte proposição:

Proposição 2.2.1. O retrato de fase de um campo vetorial polinomial quadrático planar com uma integral primeira racional de grau 2 da forma (CE) dada na Proposição (2.0.7) é topologicamente equivalente ao retrato de fase $\left(E_{1}\right)$ da Figura 2.1.

\subsection{Hipérbole}

Se a integral primeira racional é da forma $\frac{\left(x^{2}-y^{2}-1\right)}{D(x, y)}$, segue de $(2.1)$ que $a_{00}=a_{02}=$ $-1, a_{20}=1$ e $a_{10}=a_{01}=a_{11}=0$. Da primeira e segunda equação de (2.2) seguem, respectivamente, que $b_{11}=0$ e $b_{20}=-b_{02}$. Então o sistema cuja integral primeira tem a forma acima é:

$$
\begin{aligned}
& \dot{x}=P(x, y)=-b_{01}+2\left(b_{00}+b_{20}\right) y+b_{01} x^{2}+2 b_{10} x y+b_{01} y^{2} \\
& \dot{y}=Q(x, y)=b_{10}+2\left(b_{00}+b_{20}\right) x+b_{10} x^{2}+2 b_{01} x y+b_{10} y^{2}
\end{aligned}
$$

Denote por $t$ o tempo do sistema diferencial associado ao campo vetorial (2.13) e faça a mudança de variáveis $(x, y, t)=(-X, Y,-T)$ segue que 


$$
\begin{array}{lll}
x(t)=-X(-T) & \Rightarrow & \frac{d x}{d t}=\frac{-d X}{-d T}=\frac{d X}{d T} \\
y(t)=Y(-T) & \Rightarrow & \frac{d y}{d t}=\frac{d Y}{-d T}=-\frac{d Y}{d T}
\end{array}
$$

$\log \mathrm{O}$

$$
\begin{aligned}
& \dot{X}=P(-X, Y)=-b_{01}+2\left(b_{00}+b_{20}\right) Y+b_{01} X^{2}-2 b_{10} X Y+b_{01} Y^{2}, \\
& \dot{Y}=-Q(-X, Y)=-b_{10}+2\left(b_{00}+b_{20}\right) X-b_{10} X^{2}+2 b_{01} X Y-b_{10} Y^{2} .
\end{aligned}
$$

Comparando os sistemas (2.13) e (2.14) concluímos que $b_{10} \geq 0$.

Fazendo a mudança de variávies $(x, y, t)=(X,-Y,-T)$ temos

$$
\begin{array}{lll}
x(t)=X(-T) & \Rightarrow \quad & \frac{d x}{d t}=\frac{d X}{-d T}=-\frac{d X}{d T} \\
y(t)=-Y(-T) & \Rightarrow & \frac{d y}{d t}=\frac{-d Y}{-d T}=\frac{d Y}{d T}
\end{array}
$$

donde segue que

$$
\begin{aligned}
& \dot{X}=-P(X,-Y)=b_{01}+2\left(b_{00}+b_{20}\right) Y-b_{01} X^{2}+2 b_{10} X Y-b_{01} y^{2} \\
& \dot{Y}=Q(X,-Y)=b_{10}+2\left(b_{00}+b_{20}\right) X+b_{10} X^{2}-2 b_{01} X Y+b_{10} Y^{2}
\end{aligned}
$$

e podemos assuimir $b_{01} \geq 0$.

Finalmente, fazendo a mudança $(x, y, t)=(-X,-Y,-T)$ temos

$$
\begin{array}{lll}
x(t)=-X(-T) & \Rightarrow & \frac{d x}{d t}=\frac{-d X}{-d T}=\frac{d X}{d T} \\
y(t)=-Y(-T) & \Rightarrow & \frac{d y}{d t}=\frac{-d Y}{-d T}=\frac{d Y}{d T}
\end{array}
$$

e assim

$$
\begin{aligned}
& \dot{X}=P(-X,-Y)=-b_{01}-2\left(b_{00}+b_{20}\right) Y+b_{01} X^{2}+2 b_{10} X Y+b_{01} Y^{2}, \\
& \dot{Y}=Q(-X,-Y)=b_{10}-2\left(b_{00}+b_{20}\right) X+b_{10} X^{2}+2 b_{01} X Y+b_{10} Y^{2} .
\end{aligned}
$$

Note que neste caso, todos os coeficientes de (2.13) continuam iguais exceto $\left(b_{00}+b_{20}\right)$, $\operatorname{logo} b_{00}+b_{20} \geq 0$.

Em resumo, nesta seção consideramos que $b_{01} \geq 0, b_{10} \geq 0$ e $b_{00}+b_{20} \geq 0$.

Denote por 


$$
\begin{gathered}
r=b_{10}^{2}-b_{01}^{2} \\
p=b_{00}+b_{20} \\
q=p^{2}-r
\end{gathered}
$$

\section{Análise dos pontos singulares infinitos.}

Na carta $U_{1}$ o campo vetorial (2.13) é da forma:

$$
\begin{aligned}
& \dot{z}_{1}=b_{10}+b_{01} z_{1}+2 p z_{2}-b_{10} z_{1}^{2}+b_{10} z_{2}^{2}-b_{01} z_{1}^{3}-2 p z_{1}^{2} z_{2}+b_{01} z_{1} z_{2}^{2} \\
& \dot{z}_{2}=-b_{01} z_{2}-2 b_{10} z_{1} z_{2}-b_{01} z_{1}^{2} z_{2}-2 p z_{1} z_{2}^{2}+b_{01} z_{2}^{3}
\end{aligned}
$$

Os pontos singulares em $U_{1}$ são: $(-1,0),(1,0)$ e $\left(-\frac{b_{10}}{b_{01}}, 0\right)$ com matriz Jacobiana:

$$
\begin{gathered}
J X(-1,0)=\left[\begin{array}{cc}
2\left(b_{10}-b_{01}\right) & 0 \\
0 & 2\left(b_{10}-b_{01}\right)
\end{array}\right] \Rightarrow T^{2}=4 \Delta=16\left(b_{10}-b_{01}\right)^{2}, \\
J X(1,0)=\left[\begin{array}{cc}
-2\left(b_{10}+b_{01}\right) & 0 \\
0 & -2\left(b_{10}+b_{01}\right)
\end{array}\right] \Rightarrow T^{2}=4 \Delta=16\left(b_{10}+b_{01}\right)^{2}, \\
J X\left(-\frac{b_{10}}{b_{01}}, 0\right)=\left[\begin{array}{cc}
-\frac{r}{b_{01}} & -\frac{2 r p}{b_{01}^{2}} \\
0 & \frac{r}{b_{01}}
\end{array}\right] \Rightarrow \Delta=-\frac{r^{2}}{b_{01}^{2}} \text { e } T=0 .
\end{gathered}
$$

Concluimos portanto, que $(-1,0)$ é um nó estável se $T=4\left(b_{10}-b_{01}\right)<0$ e instável se $T=4\left(b_{10}-b_{01}\right)>0,(1,0)$ é um nó estável, pois $T=-4\left(b_{10}+b_{01}\right)<0$ e $\left(-\frac{b_{10}}{b_{01}}, 0\right)$ é do tipo sela se $r \neq 0$ e $b_{01} \neq 0$.

Assim, o estudo das singularidades em $U_{1}$ é dividido em quatro casos:

i) se $r \neq 0, b_{01} \neq 0$ e $-1<-\frac{b_{10}}{b_{01}} \leq 0$.

O campo vetorial em $U_{1}$ tem três singularidades: $(-1,0)$ do tipo nó estável, $(1,0)$ nó estável e $\left(-\frac{b_{10}}{b_{01}}, 0\right)$ sela.

ii) se $r \neq 0, b_{01} \neq 0$ e $-\frac{b_{10}}{b_{01}}<-1$.

O campo vetorial em $U_{1}$ tem três singularidades: $(-1,0)$ do tipo nó instável, $(1,0)$ nó estável e $\left(-\frac{b_{10}}{b_{01}}, 0\right)$ sela. 
iii) se $r \neq 0$ e $b_{01}=0$.

O campo tem apenas duas singularidades: $(-1,0)$ nó instável e $(1,0)$ nó estável.

iv) se $r=0$, então $b_{01}=b_{10}>0$. Note que se $b_{01}=b_{10}=0$ o campo vetorial (2.13) é linear.

Nesse caso, os dois pontos singulares são: $(-1,0)$ linearmente nulo e $(1,0)$ nó estável.

Para estudar o retrato de fase numa vizinhança de $(-1,0)$ primeiramente transladamoso para a origem e, em seguida, aplicamos um blow-up. Para isto, façamos a seguinte mudança de coordenadas: $Z_{1}=z_{1}+1, Z_{2}=z_{2}$ e o campo (2.17) passa ao sistema

$$
\begin{aligned}
& \dot{Z}_{1}=2 b_{10} Z_{1}^{2}+4 p Z_{1} Z_{2}-b_{10} Z_{1}^{3}-2 p Z_{1}^{2} Z_{2}+b_{10} Z_{1} Z_{2}^{2}, \\
& \dot{Z}_{2}=2 p Z_{2}^{2}-b_{10} Z_{1}^{2} Z_{2}-2 p Z_{1} Z_{2}^{2}+b_{10} Z_{2}^{3} .
\end{aligned}
$$

Aplicando o blow up, $Z_{1}=u, Z_{2}=u v$, obtemos um novo campo vetorial que é:

$$
\begin{aligned}
& \dot{u}=2 b_{10} u^{2}+4 p u^{2} v-b_{10} u^{3}-2 p u^{3} v+b_{10} u^{3} v^{2}, \\
& \dot{v}=-2 b_{10} u v-2 p u v^{2} .
\end{aligned}
$$

Dividindo $\dot{u}$ e $\dot{v}$ por $u$ temos:

$$
\begin{aligned}
& \dot{u}=2 b_{10} u+4 p u v-b_{10} u^{2}-2 p u^{2} v+b_{10} u^{2} v^{2}, \\
& \dot{v}=-2 b_{10} v-2 p v^{2} .
\end{aligned}
$$

Em $u=0$, se $p>0$ encontramos dois pontos singulares $(0,0)$ e $\left(0,-\frac{b_{10}}{p}\right)$. Se $p=0$ apenas $(0,0)$. As matrizes Jacobianas são dadas por

$$
\begin{gathered}
J X(0,0)=\left[\begin{array}{cc}
2 b_{10} & 0 \\
0 & -2 b_{10}
\end{array}\right] \Rightarrow \Delta=-4 b_{10}^{2}<0 \quad \mathrm{e} \\
J X\left(0,-\frac{b_{10}}{p}\right)=\left[\begin{array}{cc}
-2 b_{10} & 0 \\
0 & 2 b_{10}
\end{array}\right] \Rightarrow \Delta=-4 b_{10}^{2}<0 .
\end{gathered}
$$

Logo, ambas singularidades são do tipo sela. Observe Figura 2.6. 


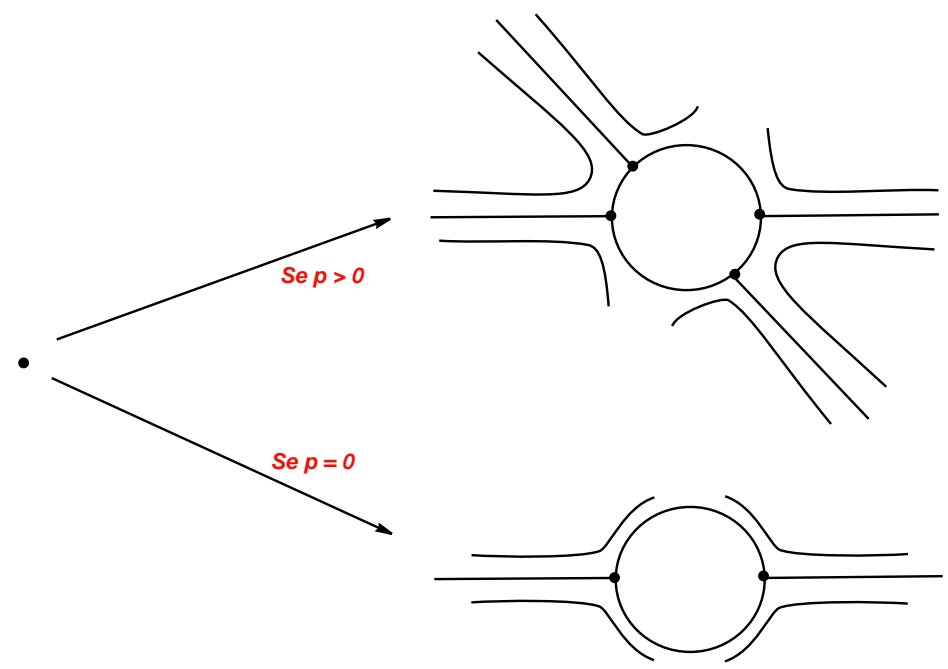

Figura 2.6: Blow up na direção $Z_{1}$.

Agora do blow up, $Z_{1}=u v, Z_{2}=v$, temos

$$
\begin{aligned}
& \dot{u}=2 b_{10} u^{2} v+2 p u v, \\
& \dot{v}=2 p v^{2}-b_{10} u^{2} v^{3}-2 p u v^{3}+b_{10} v^{3} .
\end{aligned}
$$

Dividindo $\dot{u}$ e $\dot{v}$ por $v$ temos:

$$
\begin{aligned}
& \dot{u}=2 b_{10} u^{2}+2 p u, \\
& \dot{v}=2 p v-b_{10} u^{2} v^{2}-2 p u v^{2}+b_{10} v^{2} .
\end{aligned}
$$

Em $v=0,(0,0)$ é um ponto singular com matriz Jacobiana dada por:

$$
J X(0,0)=\left[\begin{array}{cc}
2 p & 0 \\
0 & 2 p
\end{array}\right] \Rightarrow \begin{aligned}
& T=4 p>0 \\
& T^{2}=4 \Delta=16 p^{2}
\end{aligned}
$$

Logo, $(0,0)$ é singularidade do tipo nó instável se $p>0$ e, linearmente nulo, se $p=0$. Observe na Figura 2.7 um esboço do retrato de fase do campo vetorial (2.18) numa vizinhança do ponto singular $(0,0)$ para o caso $p>0$. 

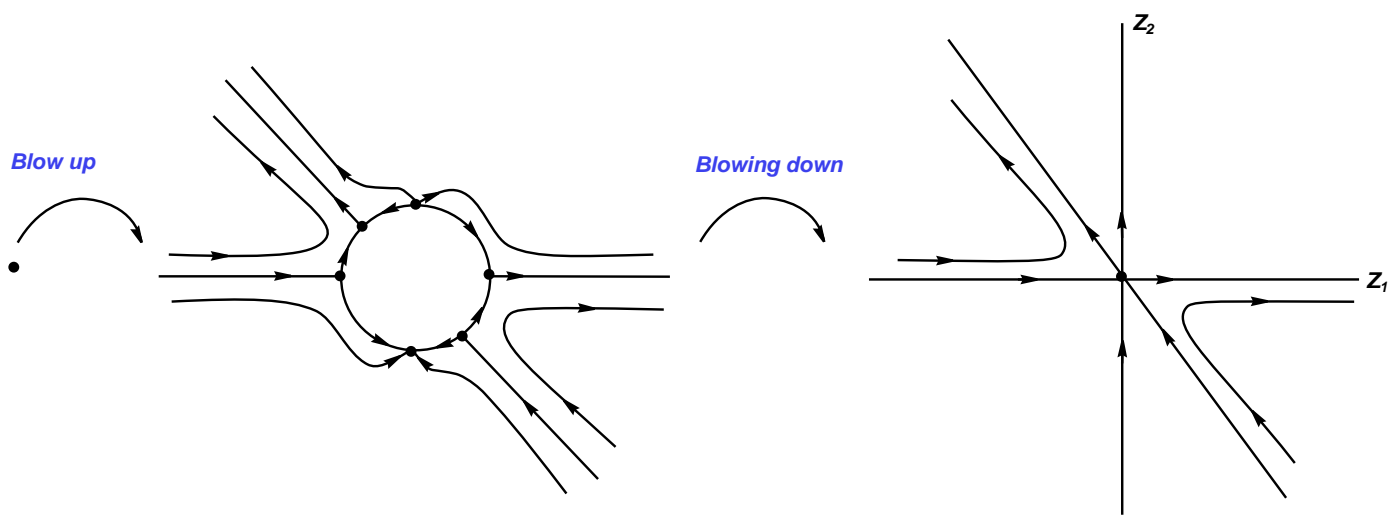

Figura 2.7: Caso $p>0$.

Agora se $p=0$ aplicamos um novo blow up para estudar o ponto singular linearmente nulo.

Fazendo a mudança de coordenadas $u=\bar{u} \bar{v}, v=\bar{v}$ e dividindo por $\bar{v}$ obtemos

$$
\begin{aligned}
& \dot{\bar{u}}=-b_{10} \bar{u}+2 b_{10} \bar{u}^{2}+b_{10} \bar{u}^{3} \bar{v}^{2} \\
& \dot{\bar{v}}=b_{10} \bar{v}-b_{10} \bar{u}^{2} \bar{v} .
\end{aligned}
$$

Logo, em $\bar{v}=0$ existem duas singularidades $(0,0)$ e $\left(\frac{1}{2}, 0\right)$ com

$$
\begin{gathered}
J X(0,0)=\left[\begin{array}{cc}
-b_{10} & 0 \\
0 & b_{10}
\end{array}\right] \Rightarrow \Delta=-b_{10}^{2}<0 \\
J X\left(\frac{1}{2}, 0\right)=\left[\begin{array}{cc}
b_{10} & 0 \\
0 & \frac{3}{4} b_{10}
\end{array}\right] \Rightarrow \begin{array}{l}
T=\frac{7}{4} b_{10}>0 \\
T^{2}=\frac{49}{16} b_{10}^{2}>4 \Delta=3 b_{10}^{2}
\end{array} .
\end{gathered}
$$

Assim, $(0,0)$ é singularidade do tipo sela e $\left(\frac{1}{2}, 0\right)$ do tipo nó instável.

Observe na Figura 2.8 o esboço do retrato de fase do campo vetorial (2.18) numa vizinhança do ponto singular $(0,0)$ para o caso $p=0$.

Portanto, para $p \geq 0,(-1,0)$ é ponto singular linearmente nulo formado por dois setores hiperbólicos separados por dois setores parabólicos. 


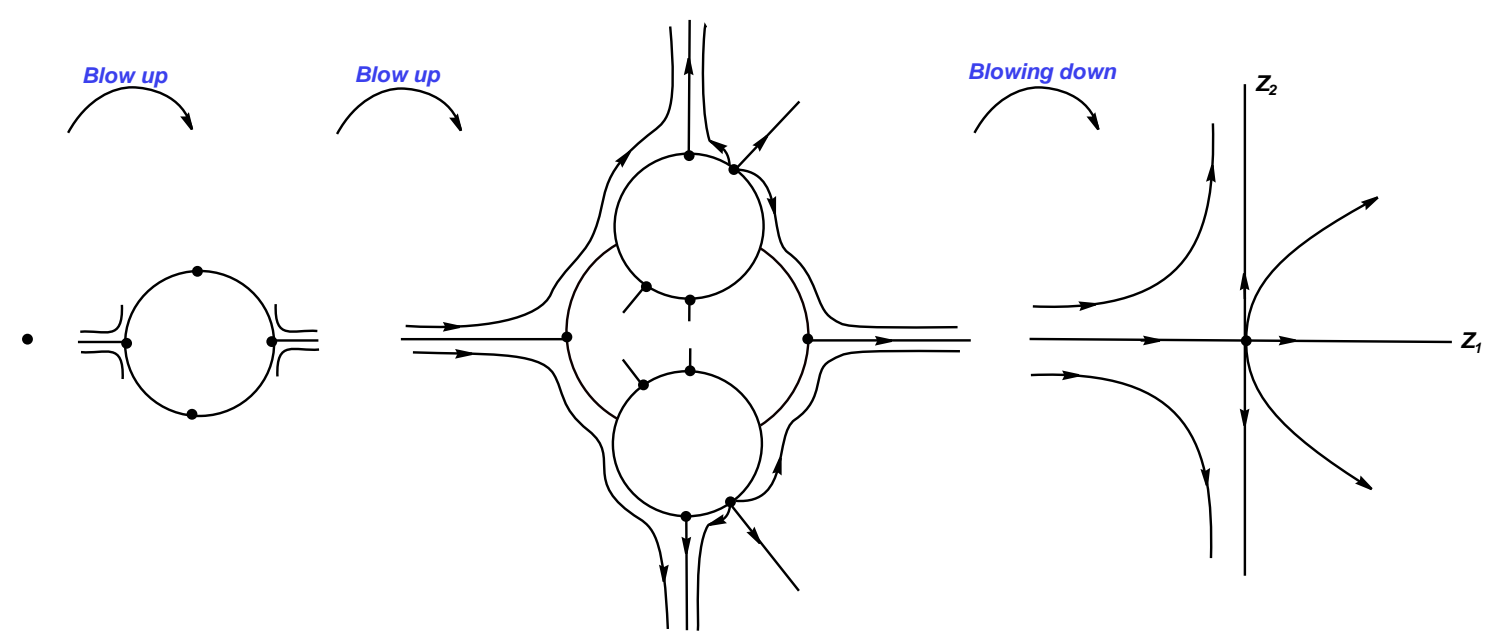

Figura 2.8: Caso $p=0$.

Finalmente, para completar o estudo dos pontos singulares infinitos estudamos a origem da carta $U_{2}$. Em $U_{2}$ o campo vetorial (2.13) é da forma:

$$
\begin{aligned}
& \dot{z_{1}}=-b_{01}-b_{10} z_{1}-2 p z_{2}+b_{01} z_{1}^{2}+b_{01} z_{2}^{2}+b_{10} z_{1}^{3}+2 p z_{1}^{2} z_{2}+b_{10} z_{1} z_{2}^{2} \\
& \dot{z_{2}}=b_{10} z_{2}+2 b_{01} z_{1} z_{2}+b_{10} z_{1}^{2} z_{2}+2 p z_{1} z_{2}^{2}+b_{10} z_{2}^{3}
\end{aligned}
$$

Se $b_{01}=0$ a origem não é singularidade do sistema.

Se $b_{01}=0$ a origem é um ponto singular com matriz Jacobiana:

$$
J X(0,0)=\left[\begin{array}{cc}
b_{10} & 2 p \\
0 & -b_{10}
\end{array}\right] \Rightarrow \Delta=-b_{10}^{2}<0
$$

e, $(0,0)$ é singularidade do tipo sela.

$\mathrm{Na}$ Figura 2.9 apresentamos um esboço do retrato de fase no infinito no disco de Poincaré. Note que e) é obtido de b) por uma translação do ponto de sela.

\section{Análise dos pontos singulares finitos.}

Se $r \neq 0$ existem 4 pontos singulares finitos, são eles

$$
\begin{gathered}
\left(x_{1}, y_{1}\right)=\left(\frac{-b_{10} p+b_{01} \sqrt{q}}{r}, \frac{b_{01} p-b_{10} \sqrt{q}}{r}\right),\left(x_{2}, y_{2}\right)=\left(\frac{-b_{10} p-b_{01} \sqrt{q}}{r}, \frac{b_{01} p+b_{10} \sqrt{q}}{r}\right), \\
\left(x_{3}, y_{3}\right)=\frac{1}{p-\sqrt{q}}\left(-b_{10}, b_{01}\right),\left(x_{4}, y_{4}\right)=\frac{1}{p+\sqrt{q}}\left(-b_{10}, b_{01}\right)
\end{gathered}
$$




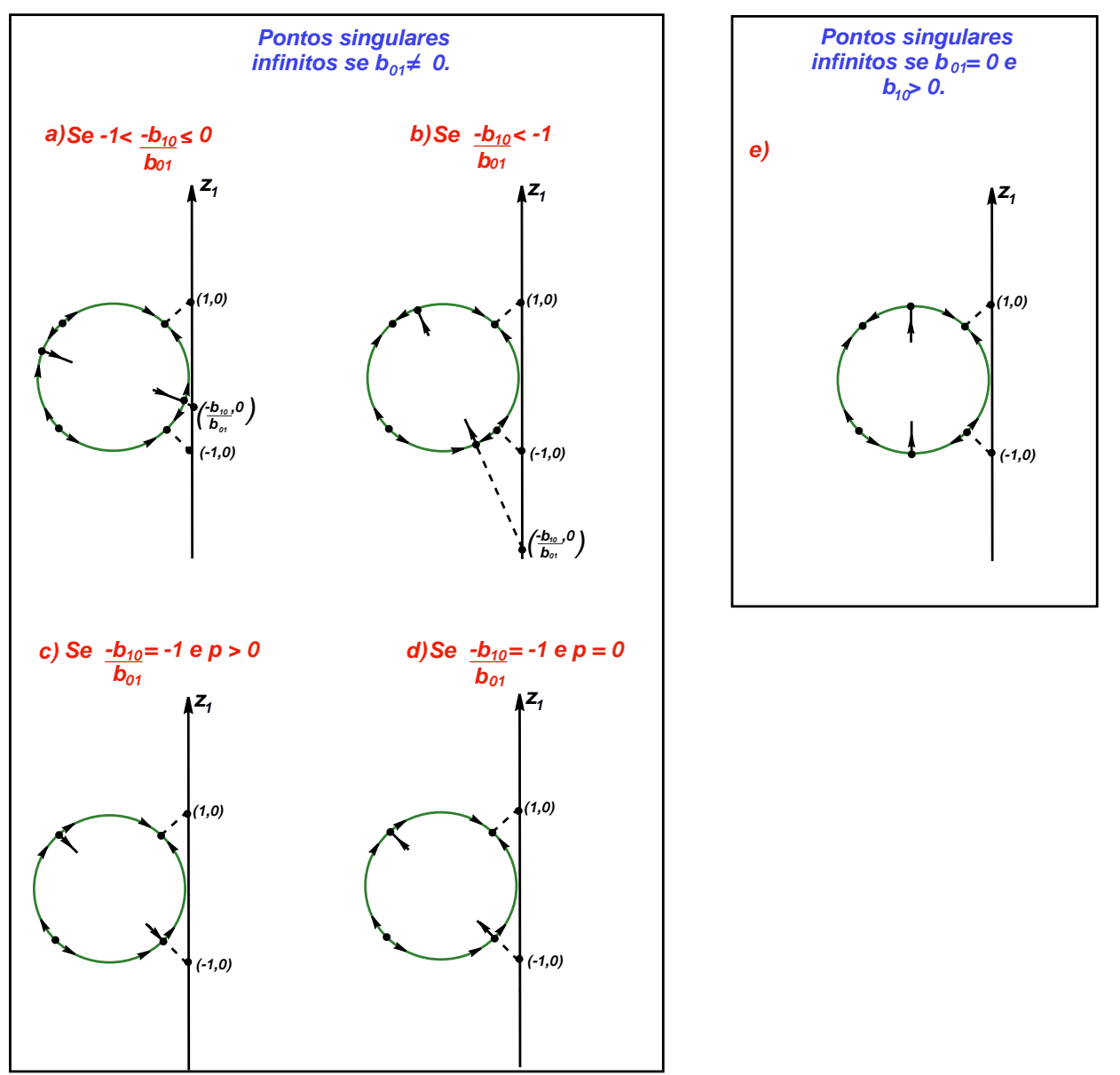

Figura 2.9: Pontos singulares infinitos para o caso $(H)$.

Temos os seguintes casos:

i) $q>0$, isto é, os quatro pontos são reais e

$$
J X\left(x_{1}, y_{1}\right)=\left[\begin{array}{cc}
2 \sqrt{q} & 0 \\
0 & 2 \sqrt{q}
\end{array}\right], \quad J X\left(x_{2}, y_{2}\right)=\left[\begin{array}{cc}
-2 \sqrt{q} & 0 \\
0 & -2 \sqrt{q}
\end{array}\right]
$$

Assim, para $\left(x_{1}, y_{1}\right)$ e $\left(x_{2}, y_{2}\right)$ temos $T^{2}=4 \Delta=16 q$. Então, $\left(x_{1}, y_{1}\right)$ é um nó instável $(T=4 \sqrt{q}>0)$ e $\left(x_{2}, y_{2}\right)$ é um nó estável $(T=-4 \sqrt{q}<0)$.

$$
J X\left(x_{3}, y_{3}\right)=\left[\begin{array}{cc}
0 & -2 \sqrt{q} \\
-2 \sqrt{q} & 0
\end{array}\right], \quad J X\left(x_{4}, y_{4}\right)=\left[\begin{array}{cc}
0 & 2 \sqrt{q} \\
2 \sqrt{q} & 0
\end{array}\right]
$$


Para $\left(x_{3}, y_{3}\right)$ e $\left(x_{4}, y_{4}\right)$ temos $\Delta=-4 q<0$, logo ambos são pontos singulares do tipo sela.

Note que $\left(x_{1}, y_{1}\right)$ e $\left(x_{2}, y_{2}\right)$ pertencem a hiperbóle invariante $x^{2}-y^{2}=1$. E temos também

$$
\begin{gathered}
x_{3}^{2}-y_{3}^{2}-1=\frac{2 \sqrt{q}}{p-\sqrt{q}}\left\{\begin{array}{c}
>0 \text { se } p-\sqrt{q}>0 \\
<0 \text { se } p-\sqrt{q}<0
\end{array},\right. \\
x_{4}^{2}-y_{4}^{2}-1=-\frac{2 \sqrt{q}}{p+\sqrt{q}}<0 .
\end{gathered}
$$

Lembrando que as curvas da hiperbóle $x^{2}-y^{2}=1$ delimitam três regiões distintas do plano, observamos que se $p-\sqrt{q}<0$ então $\left(x_{3}, y_{3}\right)$ e $\left(x_{4}, y_{4}\right)$ pertencem a mesma região e se $p-\sqrt{q}>0$ esses pontos pertencem a regiões distintas.

Suponha $p-\sqrt{q}<0^{1}, \log 0$ :

$$
x_{1}=\frac{-b_{10} p+b_{01} \sqrt{q}}{r}<0, x_{2}=\frac{-b_{10} p-b_{01} \sqrt{q}}{r}>0, y_{2}=\frac{b_{01} p+b_{10} \sqrt{q}}{r}<0
$$

e segue que $\left(x_{1}, y_{1}\right)$ e $\left(x_{2}, y_{2}\right)$ pertencem a ramos distintos da hipérbole. Além disso,

$$
x_{3}=\frac{-b_{10}}{p-\sqrt{q}} \geq 0, y_{3}=\frac{b_{01}}{p-\sqrt{q}}<0, x_{4}=\frac{-b_{10}}{p+\sqrt{q}} \leq 0 \text { e } y_{4}=\frac{b_{01}}{p+\sqrt{q}}>0 .
$$

Suponha $p-\sqrt{q}>0^{2}, \log 0$

$$
x_{1}=\frac{-b_{10} p+b_{01} \sqrt{q}}{r}<0, x_{2}=\frac{-b_{10} p-b_{01} \sqrt{q}}{r}<0, y_{2}=\frac{b_{01} p+b_{10} \sqrt{q}}{r}>0
$$

e segue que $\left(x_{1}, y_{1}\right)$ e $\left(x_{2}, y_{2}\right)$ pertencem ao mesmo ramo da hipérbole. Além disso,

$$
x_{3}=\frac{-b_{10}}{p-\sqrt{q}}<0, y_{3}=\frac{b_{01}}{p-\sqrt{q}} \geq 0, x_{4}=\frac{-b_{10}}{p+\sqrt{q}}<0 \text { e } y_{4}=\frac{b_{01}}{p+\sqrt{q}} \geq 0 .
$$

Portanto, os pontos singulares finitos estão dispostos no plano como ilustrado na Figura 2.10.

\footnotetext{
${ }^{1}$ Note que $p-\sqrt{q}<0 \Rightarrow p^{2}<q($ pois,$~ q>0) \Rightarrow r=p^{2}-q<0$. Como $r=b_{10}^{2}-b_{01}^{2}<0 \Rightarrow b_{01} \neq 0$ e $b_{10}^{2}<b_{01}^{2} \Rightarrow b_{10}<b_{01}$ para todo $b_{10} \geq 0$ e $b_{01}>0$. Logo, $b_{10} p-b_{01} \sqrt{q}<0$ e além disso, $y_{3}-y_{2}=$ $-\frac{\left(b_{01}-b_{10}\right) \sqrt{q}}{r}<0 \Rightarrow y_{3}<y_{2}$ e $y_{4}-y_{1}=-\frac{\left(-b_{01}+b_{10}\right) \sqrt{q}}{r}>0 \Rightarrow y_{4}>y_{1}$.

${ }^{2}$ Se $p-\sqrt{q}>0 \Rightarrow r>0$ e $b_{10} \neq 0$ (basta inverter os sinais das desigualdades acima). Então, $y_{3}<y_{2}$, $y_{4}>y_{1}$ e $b_{10} p-b_{01} \sqrt{q}>0$.
} 


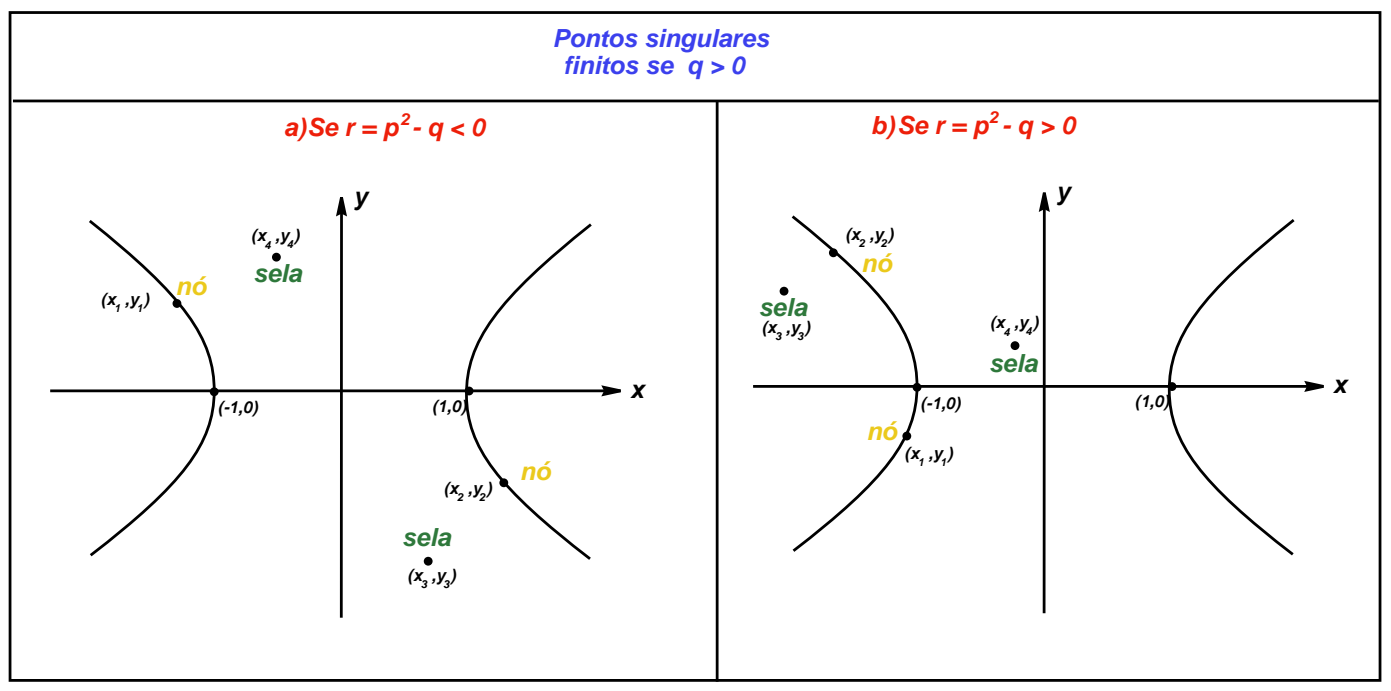

Figura 2.10: Pontos singulares finitos para o caso $(H)$ com $q>0$.

ii) $q<0$, não existem singularidades finitas.

iii) $q=0, \log 0 \neq \neq 0$, pois $r=p^{2}-q \neq 0$. Neste caso, o único ponto singular é $\frac{1}{p}\left(-b_{10}, b_{01}\right)$ com matriz Jacobiana:

$$
J X\left(\frac{1}{p}\left(-b_{10}, b_{01}\right)\right)=\left[\begin{array}{ll}
0 & 0 \\
0 & 0
\end{array}\right]
$$

e portanto, é um ponto singular linearmente nulo.

Para estudá-lo, primeiramente devemos transladá-lo para a origem. Para isto, fazemos a seguinte mudança de coordenadas: $X=x+\frac{b_{10}}{p}, Y=y-\frac{b_{01}}{p}$ e obtemos

$$
(P, Q)=\left(-b_{01}\left(X^{2}+Y^{2}\right)-2 b_{10} X Y,-b_{10}\left(X^{2}+Y^{2}\right)-2 b_{01} X Y\right) .
$$

Note que o campo vetorial acima é homogêneo. Então seu retrato de fase é um dos seis descritos na Figura 1.9.

Agora dadas $f_{1}(X, Y)=b_{10} X+b_{01} Y, f_{2}(X, Y)=Y-X$ e $f_{3}(X, Y)=X+Y$ temos que

$$
\begin{aligned}
P \frac{\partial f_{1}}{\partial X}+Q \frac{\partial f_{1}}{\partial Y} & =-2 b_{01} b_{10}\left(X^{2}+Y^{2}\right)-2 b_{10}^{2} X Y-2 b_{01}^{2} X Y \\
& =\left(-2 b_{01} X-2 b_{10} Y\right)\left(b_{10} X+b_{01} Y\right)=k_{1} f_{1}
\end{aligned}
$$




$$
\begin{aligned}
P \frac{\partial f_{2}}{\partial X}+Q \frac{\partial f_{2}}{\partial Y} & =b_{01}\left(X^{2}+Y^{2}\right)-2 b_{10} X Y-b_{01}\left(X^{2}+Y^{2}\right)-2 b_{01} X Y \\
& =\left(b_{10}(X-Y)+b_{01}(Y-X)\right)(Y-X)=k_{2} f_{2} \\
P \frac{\partial f_{3}}{\partial X}+Q \frac{\partial f_{3}}{\partial Y} & =-b_{01}\left(X^{2}+Y^{2}\right)-2 b_{10} X Y-b_{01}\left(X^{2}+Y^{2}\right)-2 b_{01} X Y \\
& =\left(-b_{10}(X+Y)-b_{01}(X+Y)\right)(X+Y)=k_{3} f_{3}
\end{aligned}
$$

onde $k_{1}(X, Y)=\left(-2 b_{01} X-2 b_{10} Y\right), k_{2}(X, Y)=\left(b_{10}(X-Y)+b_{01}(Y-X)\right) \mathrm{e}$ $k_{3}(X, Y)=\left(-b_{10}(X+Y)-b_{01}(X+Y)\right)$. Logo, $f_{1}(X, Y)=0, f_{2}(X, Y)=0$ e $f_{3}(X, Y)=0$ são retas invariantes passando pela origem. Como este campo vetorial tem no infinito duas selas e quatro nós, então seu retrato de fase é topologicamente equivalente ao dado na Figura 1.9 f). Daí segue que a origem e, portanto o ponto singular $\frac{1}{p}\left(-b_{10}, b_{01}\right)$ é formado por dois setores hiperbólicos separados por dois setores parabólicos.

Se $r=0$, então $q=p^{2}$ e $b_{10}=b_{01}$ (caso contrário, (2.13) seria linear). Se $p>0$, então o campo vetorial $(2.13)$ tem dois pontos singulares: $\frac{b_{10}}{2 p}(-1,1)$ e $\frac{1}{2 p b_{10}}\left(-b_{10}^{2}-p^{2}, b_{10}^{2}-p^{2}\right)$ com

$$
\begin{gathered}
J X\left(\frac{b_{10}}{2 p}(-1,1)\right)=\left[\begin{array}{cc}
0 & 2 p \\
2 p & 0
\end{array}\right] \Rightarrow \Delta=-4 p^{2}<0, \\
J X\left(\frac{1}{2 p b_{10}}\left(-b_{10}^{2}-p^{2}, b_{10}^{2}-p^{2}\right)\right)=\left[\begin{array}{cc}
-2 p & 0 \\
0 & -2 p
\end{array}\right] \Rightarrow \begin{array}{l}
T=-4 p<0 \\
T^{2}=4 \Delta=16 p^{2}
\end{array} .
\end{gathered}
$$

Portanto, $\left(x_{1}, y_{1}\right)=\frac{b_{10}}{2 p}(-1,1)$ é um ponto singular do tipo sela e $\left(x_{2}, y_{2}\right)=\frac{1}{2 p b_{10}}\left(-b_{10}^{2}-\right.$ $\left.p^{2}, b_{10}^{2}-p^{2}\right)$ é do tipo nó estável.

Neste caso, é importante observar que $x_{1}=-\frac{b_{10}}{2 p}<0, x_{2}=\frac{1}{2 p b_{10}}\left(-b_{10}^{2}-p^{2}\right)<0$. Além disso, temos $x_{1}-x_{2}=y_{1}-y_{2}=\frac{p}{2 b_{10}}>0$, e então $x_{1}>x_{2}$ e $y_{1}>y_{2}$.

Se $p=0$ não existem pontos singulares finitos.

Finalmente, juntando as informações obtidas para os pontos singulares infinitos e finitos, podemos fazer um esboço do retrato de fase global do campo vetorial (2.13).

Note que se $b_{01}>0$ e $-1<-\frac{b_{10}}{b_{01}} \leq 0$ então $r=b_{10}^{2}-b_{01}^{2}<0, \operatorname{logo} q=p^{2}-r>0$. Assim, para obter o retrato de fase global do campo vetorial (2.13) basta juntar as Figuras 2.9 a) e 2.10 a). Observe Figura 2.11. 


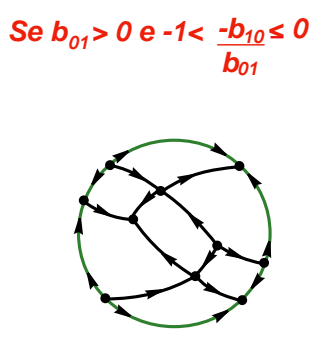

Figura 2.11: Retrato de fase no disco de Poincaré para o caso $(H)$.

Agora se $b_{01}>0$ e $-\frac{b_{10}}{b_{01}}<-1$, então $r=b_{10}^{2}-b_{01}^{2}>0$ e, existem três possibilidades: $q>0, q<0$ e $q=0$. E para cada caso o retrato de fase global do campo (2.13) é o dado nas Figuras 2.12 a), b), c) respectivamente.

$$
\text { Se } b_{01}>0 e-\frac{b_{10}}{b_{01}}<-1
$$
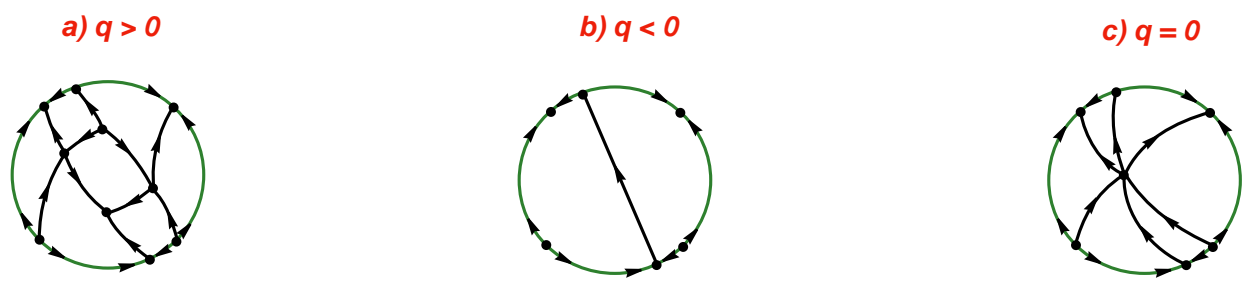

Figura 2.12: Retratos de fase no disco de Poincaré para o caso $(H)$.

Note que a Figura 2.12 a) foi obtida a partir das Figuras 2.9 b) e 2.10 b). Além disso, o retrato de fase ilustrado em a) é obtido do retrato de fase dado em Figura 2.11 por meio de uma rotação do disco e uma inversão na orientação de todas as órbitas.

Agora, se $b_{01}>0$ e $\frac{b_{10}}{b_{01}}=-1$, então $r=0$ e $q=p^{2}-r=p^{2} \geq 0$. Logo, se $p>0$ o retrato de fase é o dado pela Figura 2.13 a), e se $p=0$ pela Figura 2.13 b).

Finalmente, se $b_{01}=0$ e $b_{10}>0$, o retrato de fase global do campo vetorial (2.13) é dado pelas seguintes figuras: Figura 2.12 a) se $q>0$ (obtido a partir das Figuras 2.9 e) e 2.10 b)), Figura 2.12 b) se $q<0$ e Figura 2.12 c) se $q=0$. 
Se $\frac{-b_{10}}{b_{01}}=-1$

a) $p>0$

b) $p=0$
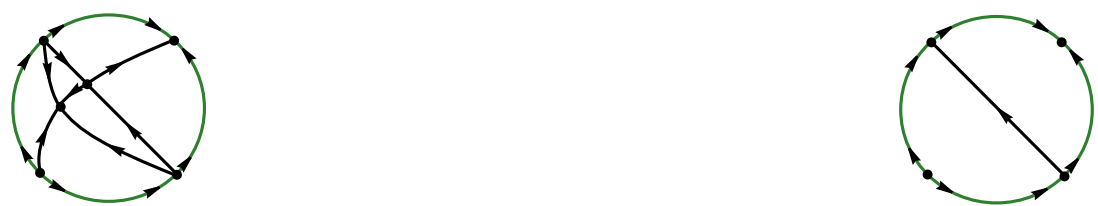

Figura 2.13: Retratos de fase no disco de Poincaré para o caso $(H)$.

Resumindo, temos o seguinte resultado:

Proposição 2.3.1. O retrato de fase de um campo vetorial polinomial quadrático planar com uma integral primeira racional de grau 2 da forma (H) dada na Proposição (2.0.7) é topologicamente equivalente aos seguintes retratos de fase da Figura 2.1:

(i) $\left(H_{1}\right)$ se $b_{01}>0 e-1<-\frac{b_{10}}{b_{01}} \leq 0$; se $q>0$ e, ou $b_{01}>0 e-\frac{b_{10}}{b_{01}}<-1$, ou $b_{01}=0 e$ $b_{10}>0$

(ii) $\left(H_{2}\right)$ se $b_{01}>0$ e $\frac{b_{10}}{b_{01}}=-1$ e $p=0$;

(iii) $\left(H_{3}\right)$ se $b_{01}>0, \frac{b_{10}}{b_{01}}=-1$ e $p>0$;

(iv) $\left(H_{4}\right)$ se $q<0$ e, ou $b_{01}>0$ e $\frac{b_{10}}{b_{01}}<-1$, ou $b_{01}=0$ e $b_{10}>0$;

(v) $\left(H_{5}\right)$ se $q=0$ e, ou $b_{01}>0$ e $\frac{b_{10}}{b_{01}}<-1$, ou $b_{01}=0$ e $b_{10}>0$.

\subsection{Duas retas complexas}

Se a integral primeira racional é da forma $\frac{\left(x^{2}+y^{2}\right)}{D(x, y)}$, segue de $(2.1)$ que $a_{02}=a_{20}=1$ e $a_{00}=a_{10}=a_{01}=a_{11}=0$. Além disso, da primeira e segunda equação de (2.2) seguem, respectivamente, que $b_{11}=0$ e $b_{20}=b_{02}$. Então o sistema cuja integral primeira tem a forma acima é:

$$
\begin{aligned}
& P=-2 b_{00} y+b_{01} x^{2}-2 b_{10} x y-b_{10} y^{2} \\
& Q=2 b_{00} x+b_{10} x^{2}+2 b_{01} x y-b_{01} y^{2}
\end{aligned}
$$


Definimos $r^{2}=b_{10}^{2}+b_{01}^{2}$. Note que $r^{2}>0$, caso contrário, o campo vetorial (2.20) seria linear.

\section{Análise dos pontos singulares infinitos.}

Na carta $U_{1}$ o campo vetorial (2.20) é da forma:

$$
\begin{aligned}
& \dot{z_{1}}=b_{10}+b_{01} z_{1}+2 b_{00} z_{2}+b_{10} z_{1}^{2}+2 b_{00} z_{1}^{2} z_{2}+b_{01} z_{1}^{3} \\
& \dot{z}_{2}=-b_{01} z_{2}+2 b_{10} z_{1} z_{2}+b_{01} z_{1}^{2} z_{2}+2 b_{00} z_{1} z_{2}^{2} .
\end{aligned}
$$

Se $b_{01} \neq 0$ existe um único ponto singular infinito em $U_{1}$ que é $\left(-\frac{b_{10}}{b_{01}}, 0\right)$, com matriz Jacobiana:

$$
J X\left(-\frac{b_{10}}{b_{01}}, 0\right)=\left[\begin{array}{cc}
\frac{r^{2}}{b_{01}} & \frac{2 b_{00} r^{2}}{b_{01}^{2}} \\
0 & -\frac{r^{2}}{b_{01}}
\end{array}\right] \Rightarrow \Delta=-\frac{r^{4}}{b_{01}^{2}}<0 .
$$

Logo, $\left(-\frac{b_{10}}{b_{01}}, 0\right)$ é ponto singular infinito do tipo sela.

Se $b_{01}=0$ não existem pontos singulares na carta $U_{1}$.

Para completar o estudo dos pontos singulares infinitos estudaremos a origem da carta $U_{2}$. Nesta carta, o campo vetorial (2.20) é da forma:

$$
\begin{aligned}
& \dot{z_{1}}=-b_{01}-b_{10} z_{1}-2 b_{00} z_{2}-b_{01} z_{1}^{2}-b_{10} z_{1}^{3}-2 b_{00} z_{1}^{2} z_{2}, \\
& \dot{z_{2}}=b_{10} z_{2}-2 b_{01} z_{1} z_{2}-b_{10} z_{1}^{2} z_{2}-2 b_{00} z_{1} z_{2}^{2} .
\end{aligned}
$$

Se $b_{01}=0$ o campo $(2.22)$ tem a origem $(0,0)$ como ponto singular do tipo sela, pois

$$
J X(0,0)=\left[\begin{array}{cc}
-b_{10} & -2 b_{00} \\
0 & b_{10}
\end{array}\right] \Rightarrow \Delta=-b_{10}^{2}<0 .
$$

Observe que os pontos singulares infinitos obtidos são os mesmos que os encontrados no caso elipse (veja Figura 2.2), isto é, independente de $b_{01}=0$ ou $b_{01} \neq 0$, temos um par de selas no infinito.

\section{Análise dos pontos singulares finitos.}

Se $b_{00} \neq 0$, existem dois pontos singulares finitos: $(0,0),-\frac{2 b_{00}}{r^{2}}\left(b_{10}, b_{01}\right)$ cujas matrizes Jacobianas são dadas abaixo: 


$$
\begin{gathered}
J X(0,0)=\left[\begin{array}{cc}
0 & -2 b_{00} \\
2 b_{00} & 0
\end{array}\right] \Rightarrow \Delta=4 b_{00}^{2}>0 \text { e } T=0 . \\
J X\left(-\frac{2 b_{00}}{r^{2}}\left(b_{10}, b_{01}\right)\right)=\left[\begin{array}{cc}
0 & 2 b_{00} \\
-2 b_{00} & 0
\end{array}\right] \Rightarrow \Delta=4 b_{00}^{2}>0 \text { e } T=0 .
\end{gathered}
$$

Então, ambos são pontos singulares do tipo centro ou foco fraco. Como já vimos em outros casos, da existência da integral primeira de grau 2, concluímos que ambos são centros.

Lembrando que no infinito temos apenas um par de selas, segue que se $b_{00} \neq 0$, o retrato de fase do campo vetorial (2.20) é dado pela Figura 2.3.

Se $b_{00}=0$, o único ponto singular é $(0,0)$, o qual é linearmente nulo, isto é, todas as entradas de sua matriz Jacobiana são nulas. Neste caso, o campo vetorial (2.20) é da forma:

$$
\begin{aligned}
& P=b_{01} x^{2}-2 b_{10} x y-b_{10} y^{2}, \\
& Q=b_{10} x^{2}+2 b_{01} x y-b_{01} y^{2},
\end{aligned}
$$

o qual é homogêneo. Assim, o retrato de fase do campo (2.23) numa vizinhança de $(0,0)$ é um dos seis retratos descritos na Figura 1.9.

Além disso, dada $f(x, y)=\left(b_{10} x+b_{01} y\right)\left(x^{2}+y^{2}\right)$ segue que

$$
\begin{aligned}
P \frac{\partial f}{\partial x}+Q \frac{\partial f}{\partial y} & =2 b_{01} b_{10}\left(x^{2}-y^{2}\right)-2 b_{10}^{2} x y+2 b_{01}^{2} x y \\
& =\left(2 b_{01} x-2 b_{10} y\right)\left(b_{10} x+b_{01} y\right)=k f
\end{aligned}
$$

onde $k(x, y)=2 b_{01} x-2 b_{10} y$. Logo, $f(x, y)=0$ é reta invariante passando pela origem. Juntando a isto, o fato que o campo vetorial tem no infinito apenas um par de selas, da Figura 1.9 concluímos que o retrato de fase do campo vetorial (2.20) neste caso é o dado na Figura 1.9 a).

Resumidamente, temos:

Proposição 2.4.1. O retrato de fase de um campo vetorial polinomial quadrático planar com uma integral primeira racional de grau 2 da forma (p) dada na Proposição (2.0.7) é topologicamente equivalente aos seguintes retratos de fase da Figura 2.1: 
(i) $\left(E_{1}\right)$ se $b_{00} \neq 0$;

(ii) $\left(E_{3}\right)$ se $b_{00}=0$.

\subsection{Duas retas reais com intersecção}

Se a integral primeira racional é da forma $\frac{x y}{D(x, y)}$, segue de $(2.1)$ que $a_{00}=a_{02}=a_{20}=$ $a_{01}=a_{10}=0$ e $a_{11}=1$. Além disso, da primeira e terceira equação de (2.2) seguem, respectivamente, que $b_{20}=b_{02}=0$. Então o sistema cuja integral primeira tem a forma acima é:

$$
\begin{aligned}
& P=-x\left(b_{00}+b_{10} x\right), \\
& Q=y\left(b_{00}+b_{01} y\right) .
\end{aligned}
$$

Denotaremos por $t$ o tempo do sistema diferencial associado ao campo vetorial (2.24). Fazendo a mudança de variáveis $(x, y, t)=(-X, Y, T)$ temos

$$
\begin{array}{cccc}
x(t)=-X(T) & \Rightarrow & \frac{d x}{d t}=-\frac{d X}{d T} \\
y(t)=Y(T) & \Rightarrow & \frac{d y}{d t}=\frac{d Y}{d T}
\end{array}
$$

Logo,

$$
\begin{aligned}
& \dot{X}=P=-X\left(b_{00}-b_{10} X\right) \\
& \dot{Y}=Q=Y\left(b_{00}+b_{01} Y\right) .
\end{aligned}
$$

Observe que obtemos outro campo vetorial da forma (2.24) com o mesmo $b_{01}$ e com $-b_{10}$ ao invés de $b_{10}$. Então, podemos assumir que $b_{10} \geq 0$.

Agora fazendo a mudança de variávies $(x, y, t)=(-X,-Y,-T)$ temos

$$
\begin{array}{lll}
x(t)=-X(-T) & \Rightarrow & \frac{d x}{d t}=\frac{-d X}{-d T}=\frac{d X}{d T} \\
y(t)=-Y(-T) & \Rightarrow & \frac{d y}{d t}=\frac{-d Y}{-d T}=\frac{d Y}{d T}
\end{array}
$$

$\mathrm{e}$

$$
\begin{aligned}
& \dot{X}=P=-X\left(-b_{00}+b_{10} X\right), \\
& \dot{Y}=Q=Y\left(-b_{00}+b_{01} Y\right) .
\end{aligned}
$$

Assim, podemos assumir $b_{00} \geq 0$.

Ainda, fazendo a mudança $(x, y, t)=(X,-Y, T)$ temos 


$$
\begin{array}{ccc}
x(t)=X(T) & \Rightarrow & \frac{d x}{d t}=\frac{d X}{d T} \\
y(t)=-Y(T) & \Rightarrow & \frac{d y}{d t}=-\frac{d Y}{d T}
\end{array}
$$

e segue que

$$
\begin{aligned}
& \dot{X}=P=-X\left(b_{00}+b_{10} X\right), \\
& \dot{Y}=Q=Y\left(b_{00}-b_{01} Y\right) .
\end{aligned}
$$

Note que neste caso, todos os coeficientes de (2.24) continuam iguais exceto $b_{01}$, que muda de sinal e, por isso, podemos assumir $b_{01} \geq 0$.

Finalmente, da mudança de variáveis $(x, y, t)=(Y, X,-T)$ obtemos

$$
\begin{array}{lll}
x(t)=Y(-T) & \Rightarrow & \frac{d x}{d t}=-\frac{d Y}{d T} \\
y(t)=X(-T) & \Rightarrow & \frac{d y}{d t}=-\frac{d X}{d T}
\end{array}
$$

o que implica em:

$$
\begin{aligned}
& \dot{X}=P=-X\left(b_{00}+b_{01} X\right), \\
& \dot{Y}=Q=Y\left(b_{00}+b_{10} Y\right) .
\end{aligned}
$$

isto é, tal mudança troca $b_{10}$ com $b_{01}$. Além disso, como $b_{10}$ e $b_{01}$ não podem se anular simultâneamente (caso contrário, o campo vetorial seria linear), sem perda de generalidade, podemos assumir que $b_{10}>0$.

Em resumo, nesta seção assumiremos que $b_{01} \geq 0, b_{10}>0$ e $b_{00} \geq 0$.

\section{Análise dos pontos singulares infinitos.}

Na carta $U_{1}$ o campo vetorial (2.24) é da forma:

$$
\begin{aligned}
& \dot{z}_{1}=b_{10} z_{1}+b_{01} z_{1}^{2}+2 b_{00} z_{1} z_{2}, \\
& \dot{z}_{2}=b_{10} z_{2}+b_{00} z_{2}^{2} .
\end{aligned}
$$

Se $b_{01} \neq 0$ existem dois pontos singulares infinitos: $(0,0)$ e $\left(-\frac{b_{10}}{b_{01}}, 0\right)$, com:

$$
\begin{gathered}
J X(0,0)=\left[\begin{array}{cc}
b_{10} & 0 \\
0 & b_{10}
\end{array}\right] \Rightarrow \begin{array}{c}
T=2 b_{10}>0 \\
T^{2}=4 \Delta=4 b_{10}^{2}
\end{array}, \\
J X\left(-\frac{b_{10}}{b_{01}}, 0\right)=\left[\begin{array}{cc}
-b_{10} & -\frac{2 b_{00} b_{10}}{b_{01}} \\
0 & b_{10}
\end{array}\right] \Rightarrow \Delta=-b_{10}^{2}<0 .
\end{gathered}
$$


Logo, $(0,0)$ é ponto singular do tipo nó instável e $\left(-\frac{b_{10}}{b_{01}}, 0\right)$ do tipo sela.

Se $b_{01}=0$ a origem $(0,0)$ é o único ponto singular em $U_{1}$ e, como já vimos é do tipo nó instável.

Em $U_{2}$ o campo vetorial (2.24) é da forma:

$$
\begin{aligned}
& \dot{z}_{1}=-b_{01} z_{1}-b_{10} z_{1}^{2}-2 b_{00} z_{1} z_{2}, \\
& \dot{z_{2}}=-b_{01} z_{2}-b_{00} z_{2}^{2} .
\end{aligned}
$$

Assim, se $b_{01} \neq 0,(0,0)$ é ponto singular com matriz Jacobiana:

$$
J X(0,0)=\left[\begin{array}{cc}
-b_{01} & 0 \\
0 & -b_{01}
\end{array}\right] \Rightarrow \begin{aligned}
& T=-2 b_{01}<0 \\
& T^{2}=4 \Delta=4 b_{01}^{2}
\end{aligned}
$$

portanto, $(0,0)$ é um nó estável.

Agora se $b_{01}=0$, o campo vetorial $(2.30)$ é homogêneo, tendo a origem $(0,0)$ como ponto singular linearmente nulo.

Suponha $b_{00} \neq 0$. Utilizaremos a técnica do blow up para estudar este ponto.

Iniciamos aplicando o blow up $z_{1}=u, z_{2}=u v$ e obtemos o novo campo vetorial:

$$
\begin{aligned}
& \dot{u}=-b_{10} u^{2}-2 b_{00} u^{2} v, \\
& \dot{v}=b_{10} u v+b_{00} u v^{2} .
\end{aligned}
$$

Dividindo $\dot{u}$ e $\dot{v}$ por $u$ temos:

$$
\begin{aligned}
& \dot{u}=-b_{10} u-2 b_{00} u v, \\
& \dot{v}=b_{10} v+b_{00} v^{2} .
\end{aligned}
$$
com:

Este campo vetorial tem em $u=0$ dois pontos singulares do tipo sela, $(0,0)$ e $\left(0,-\frac{b_{10}}{b_{00}}\right)$

$$
J X(0,0)=\left[\begin{array}{cc}
-b_{10} & 0 \\
0 & b_{10}
\end{array}\right] \Rightarrow \Delta=-b_{10}^{2}<0
$$

$\mathrm{e}$

$$
J X\left(0,-\frac{b_{10}}{b_{00}}\right)=\left[\begin{array}{cc}
b_{10} & 0 \\
0 & -b_{10}
\end{array}\right] \Rightarrow \Delta=-b_{10}^{2}<0
$$


Aplicando o blow up, $z_{1}=u v, z_{2}=v$ encontramos:

$$
\begin{aligned}
& \dot{u}=-b_{10} u^{2} v-b_{00} u v, \\
& \dot{v}=-b_{00} v^{2} .
\end{aligned}
$$

Dividindo $\dot{u}$ e $\dot{v}$ por $v$ temos:

$$
\begin{aligned}
& \dot{u}=-b_{10} u^{2}-b_{00} u, \\
& \dot{v}=-b_{00} v .
\end{aligned}
$$

Nesta direção, obtemos $(0,0)$ como ponto singular do tipo nó estável, pois

$$
J X(0,0)=\left[\begin{array}{cc}
-b_{00} & 0 \\
0 & -b_{00}
\end{array}\right] \Rightarrow \begin{gathered}
T=-2 b_{00}<0 \\
T^{2}=4 \Delta=4 b_{00}^{2}
\end{gathered}
$$

Para desenhar o retrato de fase numa vizinhança de $(0,0)$ basta se unir as informações encontradas nas direções $z_{1}$ e $z_{2}$, veja Figura 2.14 .

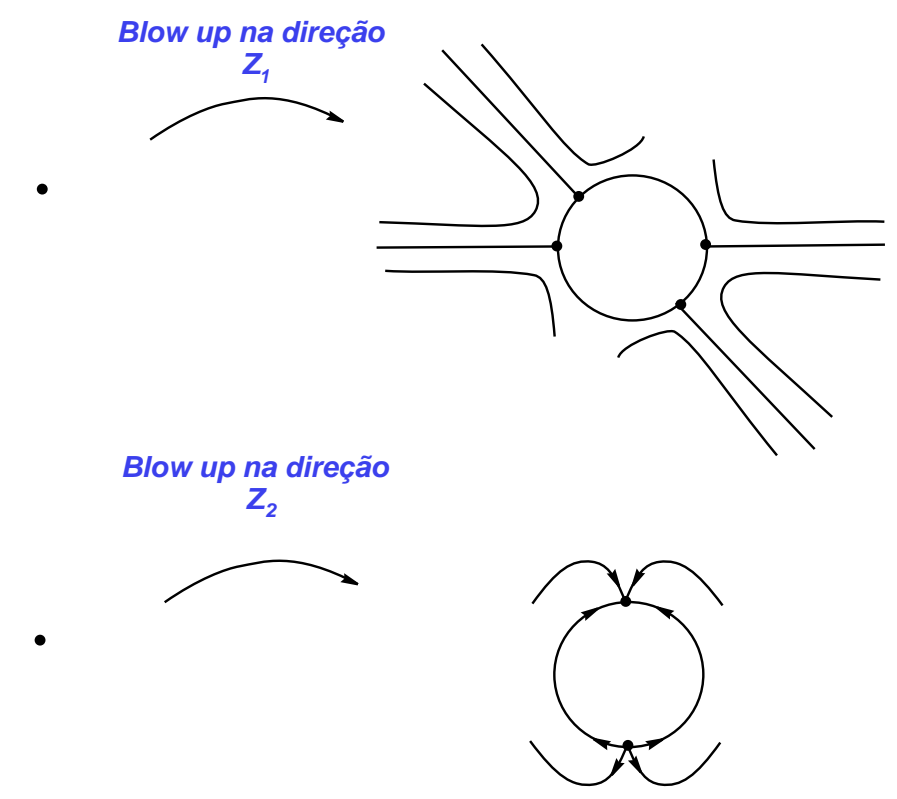

Figura 2.14: Blow ups nas direções $Z_{1}$ e $Z_{2}$.

Fazendo um blowing down, obtemos que o retrato de fase local deste campo vetorial em $(0,0)$ é formado por dois setores hiperbólicos separados por dois setores parabólicos, veja Figura 2.15. 

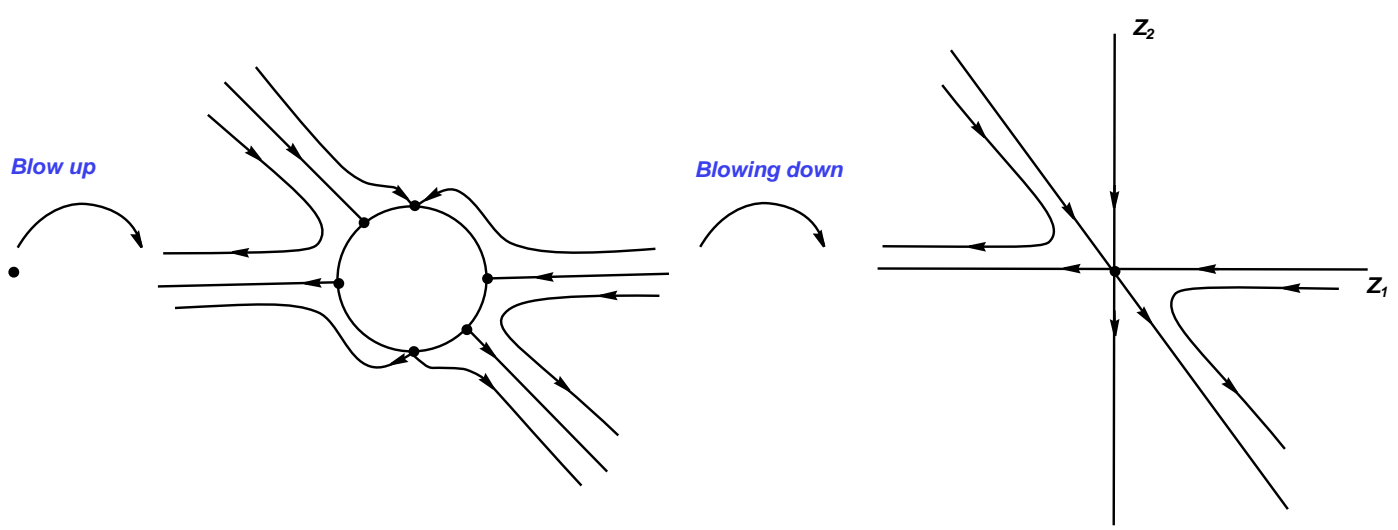

Figura 2.15: Blow up para o caso $b_{01}=0$ e $b_{00} \neq 0$.

Agora se $b_{01}=0$ e $b_{00}=0$ o campo vetorial (2.24) é da forma:

$$
\begin{aligned}
& P=-b_{10} x^{2}, \\
& Q=0 .
\end{aligned}
$$

Dividindo $P$ e $Q$ por $x$ obtemos o campo linear:

$$
\begin{aligned}
& P=-b_{10} x, \\
& Q=0 .
\end{aligned}
$$

Portanto, não consideraremos este caso.

Na Figura 2.16 apresentamos um esboço de como desenhar os pontos singulares infinitos no disco de Poincaré para os casos considerados.

\section{Análise dos pontos singulares finitos.}

Se $b_{00} b_{01} \neq 0$, existem quatro pontos singulares finitos, são eles: $(0,0),\left(0,-\frac{b_{00}}{b_{01}}\right),\left(-\frac{b_{00}}{b_{01}}, 0\right)$ $\mathrm{e}\left(-\frac{b_{00}}{b_{01}},-\frac{b_{00}}{b_{01}}\right) \mathrm{com}$ :

$$
J X(0,0)=\left[\begin{array}{cc}
-b_{00} & 0 \\
0 & b_{00}
\end{array}\right] \quad \text { e } \quad J X\left(-\frac{b_{00}}{b_{01}},-\frac{b_{00}}{b_{01}}\right)=\left[\begin{array}{cc}
b_{00} & 0 \\
0 & -b_{00}
\end{array}\right]
$$

Para $(0,0)$ e $\left(-\frac{b_{00}}{b_{01}},-\frac{b_{00}}{b_{01}}\right)$ temos $\Delta=-b_{00}^{2}<0$, logo ambos são pontos singulares do tipo sela. Além disso, 

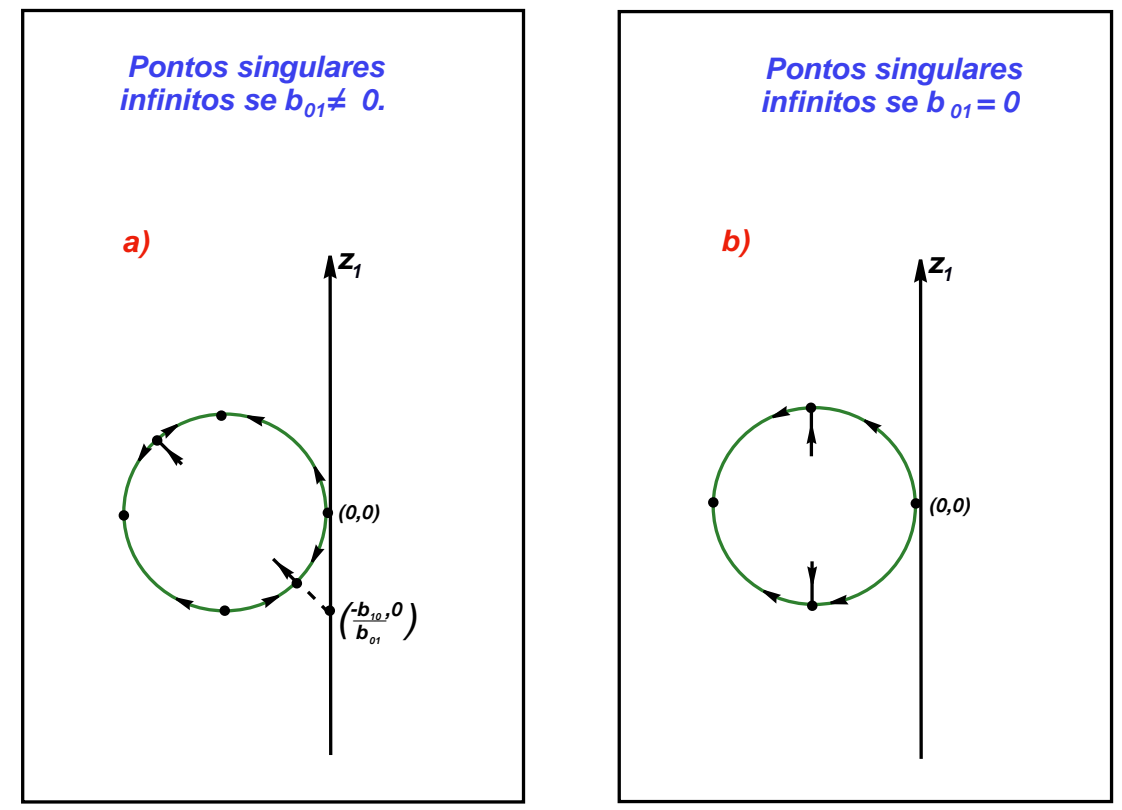

Figura 2.16: Pontos singulares infinitos para o caso $(L V)$.

$$
J X\left(0,-\frac{b_{00}}{b_{01}}\right)=\left[\begin{array}{cc}
-b_{00} & 0 \\
0 & -b_{00}
\end{array}\right] \quad J X\left(-\frac{b_{00}}{b_{01}}, 0\right)=\left[\begin{array}{cc}
b_{00} & 0 \\
0 & b_{00}
\end{array}\right]
$$

então para $\left(0,-\frac{b_{00}}{b_{01}}\right)$ e $\left(-\frac{b_{00}}{b_{01}}, 0\right)$ temos $T^{2}=4 \Delta=4 b_{00}^{2}$, logo são respectivamente nó estável $\left(T=-2 b_{00}\right)$ e instável $\left(T=2 b_{00}\right)$. Note que os pontos singulares finitos estão dispostos no plano como ilustrado na Figura 2.17.

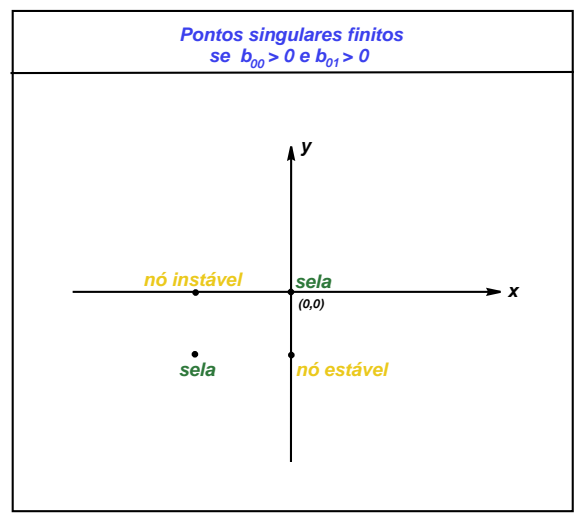

Figura 2.17: Pontos singulares finitos para o caso $(L V)$ com $b_{00}>0$ e $b_{01}>0$. 
Suponha $b_{00}=0$. Neste caso, basta considerarmos $b_{01} \neq 0$.

Temos que $(0,0)$ é o único ponto singular finito e é linearmente nulo. Neste caso, o campo vetorial (2.24) é da forma

$$
\begin{aligned}
& P=-b_{10} x^{2}, \\
& Q=b_{01} y^{2},
\end{aligned}
$$

o qual é homogêneo. Assim, seu retrato de fase é um dos seis retratos descritos na Figura 1.9 .

Ainda, dadas $f_{1}(x, y)=b_{10} x+b_{01} y, f_{2}(x, y)=x$ e $f_{3}(x, y)=y$ segue que:

$$
\begin{aligned}
P \frac{\partial f_{1}}{\partial x}+Q \frac{\partial f_{1}}{\partial x} & =-b_{10}^{2} x^{2}+b_{01}^{2} y^{2} \\
& =\left(-b_{10} x+b_{01} y\right)\left(b_{10} x+b_{01} x\right)=k_{1} f_{1}
\end{aligned},
$$

onde $k_{1}(x, y)=-b_{10} x+b_{01} y, k_{2}(x, y)=-b_{10} x$ e $k_{3}(x, y)=b_{01} y$. Logo, $f_{1}(X, Y)=0$, $f_{2}(X, Y)=0$ e $f_{3}(X, Y)=0$ são retas invariantes passando pela origem. Junto com o fato que este campo vetorial tem dois pares de nós e um par de selas no infinito para $b_{01} \neq 0$, segue que o retrato de fase deste campo é topologicamente equivalente ao dado na Figura $1.9 \mathrm{f})$.

Agora suponha $b_{00} \neq 0$. Se $b_{01}=0$ os pontos singulares são: $(0,0),\left(-\frac{b_{00}}{b_{10}}, 0\right)$, com

$$
\begin{gathered}
J X(0,0)=\left[\begin{array}{cc}
-b_{00} & 0 \\
0 & b_{00}
\end{array}\right] \Rightarrow \Delta=-b_{00}^{2}<0, \\
J X\left(-\frac{b_{00}}{b_{10}}, 0\right)=\left[\begin{array}{cc}
b_{00} & 0 \\
0 & b_{00}
\end{array}\right] \Rightarrow \begin{array}{l}
T=2 b_{00}>0 \\
T^{2}=4 \Delta=4 b_{00}^{2}
\end{array} .
\end{gathered}
$$

Assim, $(0,0)$ é do tipo sela e $\left(-\frac{b_{00}}{b_{10}}, 0\right)$ é do tipo nó instável. 
Reunindo as informações obtidas para os pontos singulares finitos e infinitos obtemos o retrato de fase global do campo (2.24).

Se $b_{01}>0$ e $b_{00}>0$ basta unir as Figuras 2.16 a) e 2.17 e obtemos o retrato de fase ilustrado na Figura 2.18.

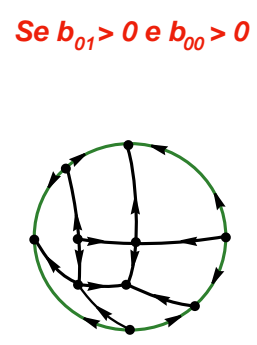

Figura 2.18: Retrato de fase no disco de Poincaré para o caso $(L V)$.

Note que o retrato de fase apresentado na Figura 2.18 pode ser obtido do retrato de fase dado na Figura 2.11 por meio de uma rotação do disco de Poincaré.

Se $b_{01}>0$ e $b_{00}=0$ o retrato de fase é dado pela Figura 2.19.

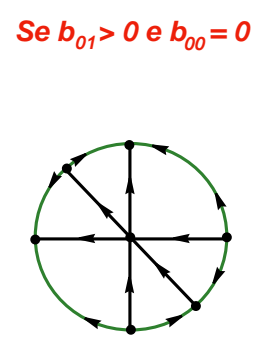

Figura 2.19: Retrato de fase no disco de Poincaré para o caso $(L V)$.

Note que o retrato de fase obtido na Figura 2.19 é topologicamente equivalente ao dado na Figura $2.12 \mathrm{c}$ ).

Se $b_{01}=0$ e $b_{00}>0$ o retrato de fase é o dado pela Figura 2.20, a qual foi obtida acrescentando na Figura 2.16 b) os dois pontos singulares finitos: uma sela e um nó instável.

Observe que o retrato de fase apresentado na Figura 2.20 é obtido do retrato de fase dado na Figura 2.13 a) por uma inversão do tempo. 
$\operatorname{Se} b_{01}=0$ e $b_{00}>0$

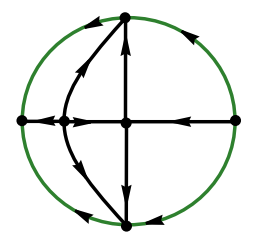

Figura 2.20: Retrato de fase no disco de Poincaré para o caso $(L V)$.

Resumidamente, temos:

Proposição 2.5.1. O retrato de fase de um campo vetorial polinomial quadrático planar com uma integral primeira racional de grau 2 da forma ( $L V$ ) dada na Proposição (2.0.7) é topologicamente equivalente aos seguintes retratos de fase da Figura 2.1:

(i) $\left(H_{1}\right)$ se $b_{01}>0$ e $b_{00}>0$;

(ii) $\left(H_{3}\right)$ se $b_{01}=0$ e $b_{00}>0$;

(iii) $\left(H_{5}\right)$ se $b_{01}>0$ e $b_{00}=0$.

\subsection{Parábola}

Se a integral primeira racional é da forma $\frac{x^{2}-y}{D(x, y)}$, segue de $(2.1)$ que $a_{00}=a_{10}=a_{02}=$ $a_{11}=0, a_{20}=1$ e $a_{01}=-1$. Além disso, da primeira e segunda equação de (2.2) seguem, respectivamente, que $b_{11}=b_{02}=0$. Então o sistema cuja integral primeira tem a forma acima é:

$$
\begin{aligned}
& P=b_{00}+b_{10} x+\left(b_{01}+b_{20}\right) x^{2} \\
& Q=2 b_{00} x+b_{10} y+b_{10} x^{2}+2\left(b_{01}+b_{20}\right) x y .
\end{aligned}
$$

Definamos $p=b_{01}+b_{20}$ e $q=b_{10}^{2}-4 b_{00} p$. Trabalhando como na Seção 2.5 podemos assumir que $p \geq 0$. 


\section{Análise dos pontos singulares infinitos.}

Na carta $U_{1}$ o campo vetorial (2.34) é da forma:

$$
\begin{aligned}
& \dot{z}_{1}=b_{10}+p z_{1}+2 b_{00} z_{2}-b_{00} z_{1} z_{2}^{2}, \\
& \dot{z}_{2}=-p z_{2}-b_{10} z_{2}^{2}-b_{00} z_{2}^{3} .
\end{aligned}
$$

Se $p>0$ existe um único ponto singular infinito: $\left(-\frac{b_{10}}{p}, 0\right)$, com:

$$
J X\left(-\frac{b_{10}}{p}, 0\right)=\left[\begin{array}{cc}
p & 2 b_{00} \\
0 & -p
\end{array}\right] \Rightarrow \Delta=-p^{2}<0
$$

portanto, o ponto singular é uma sela.

Se $p=0$ e $b_{10} \neq 0$ então não existem pontos singulares infinitos em $U_{1}$.

Notamos que $p=b_{10}=0$ implica que (2.34) é campo linear.

$\mathrm{Na}$ carta $U_{2}$ temos o sistema:

$$
\begin{aligned}
& \dot{z_{1}}=-p z_{1}^{2}+b_{00} z_{2}^{2}-b_{10} z_{1}^{3}-2 b_{00} z_{1}^{2} z_{2}, \\
& \dot{z_{2}}=-2 p z_{1} z_{2}-b_{10} z_{2}^{2}-b_{10} z_{1}^{2} z_{2}-2 b_{00} z_{1} z_{2}^{2},
\end{aligned}
$$

tendo $(0,0)$ ponto singular linearmente nulo.

Para estudar o retrato de fase numa vizinhança de $(0,0)$ façamos um blow up direcional.

Iniciamos aplicando o blow up $z_{1}=u, z_{2}=u v$ e obtemos

$$
\begin{aligned}
& \dot{u}=-p u^{2}-b_{10} u^{3}-2 b_{00} u^{3} v+b_{00} u^{2} v^{2}, \\
& \dot{v}=-p u v-b_{10} u v^{2}-b_{00} u v^{3} .
\end{aligned}
$$

Dividindo a expressão acima por $u$ segue que

$$
\begin{aligned}
& \dot{u}=-p u-b_{10} u^{2}-2 b_{00} u^{2} v+b_{00} u v^{2} \\
& \dot{v}=-p v-b_{10} v^{2}-b_{00} v^{3}
\end{aligned}
$$

Em $u=0$, o estudo das singularidades de (2.37) deve ser dividido em casos de acordo com o valor de $p$ e $q$.

Antes desse estudo, vamos verificar se $(0,0)$ é singularidade na outra direção, isto é, na direção $z_{2}$.

Fazendo a mudança de coordenadas $z_{1}=u v, z_{2}=v$ e dividindo por $v$ obtemos 


$$
\begin{aligned}
& \dot{u}=p u^{2}+b_{00}-b_{10} u, \\
& \dot{v}=-2 p u v-b_{10} v-b_{10} u^{2} v^{2}-b_{00} u v^{2} .
\end{aligned}
$$

É imediato que em $v=0,(0,0)$ é singularidade do sistema acima se e, somente se, $b_{00}=0$. E neste caso, temos que

$$
J X(0,0)=\left[\begin{array}{cc}
b_{10} & 0 \\
0 & -b_{10}
\end{array}\right] \Rightarrow \Delta=-b_{10}^{2}<0 .
$$

Logo, se $b_{00}=0,(0,0)$ é singularidade do tipo sela.

A seguir, faremos o estudo das singularidades de (2.37) em $u=0$.

- $p>0, q>0$ e $b_{00} \neq 0$. Neste caso, em $u=0$ o campo vetorial (2.37) tem três pontos singulares: $\left(x_{1}, y_{1}\right)=(0,0),\left(x_{2}, y_{2}\right)=\left(0, \frac{-b_{10}+\sqrt{q}}{2 b_{00}}\right)$ e $\left(x_{3}, y_{3}\right)=\left(0, \frac{-b_{10}-\sqrt{q}}{2 b_{00}}\right)$ com

$$
\begin{gathered}
J X\left(x_{1}, y_{1}\right)=\left[\begin{array}{cc}
-p & 0 \\
0 & -p
\end{array}\right] \Rightarrow \begin{array}{c}
T=-2 p<0 \\
T^{2}=4 \Delta=4 p^{2}
\end{array} \quad \mathrm{e} \\
J X\left(x_{2}, y_{2}\right)=J X\left(x_{3}, y_{3}\right)=\left[\begin{array}{cc}
\frac{\left(q-b_{10} \sqrt{q}\right)}{2 b_{00}} & 0 \\
0 & \frac{\left(-q+b_{10} \sqrt{q}\right)}{2 b_{00}}
\end{array}\right] \Rightarrow \\
\Delta=-\frac{\left(b_{10}-\sqrt{q}\right)^{2} q}{4 b_{00}^{2}}<0
\end{gathered}
$$

Então, $\left(x_{1}, y_{1}\right)$ é do tipo nó estável e $\left(x_{2}, y_{2}\right),\left(x_{3}, y_{3}\right)$ são selas. Note que $q-b_{10} \sqrt{q} \neq 0$, caso contrário, teríamos $q=0$ ou $q=b_{10}^{2}$ o que implicaria em $b_{00} p=0$.

Se $b_{00}>0$ e $b_{10}>0$ as duas selas estão sob eixo $v$ negativo (veja Figura 2.21). Se $b_{00}>0$ e $b_{10}<0$ ambas estão sob eixo $v$ positivo (veja Figura 2.22). Se $b_{00}<0$ as duas selas estão separadas pelo nó (veja Figura 2.23).

Lembrando que para este caso $(0,0)$ não é singularidade na direção $z_{2}$ e aplicando um blowing down temos que a origem de (2.36) é formada por dois setores hiperbólicos separados por dois setores parabólicos como mostrado nas Figuras 2.21, 2.22 e 2.23. 

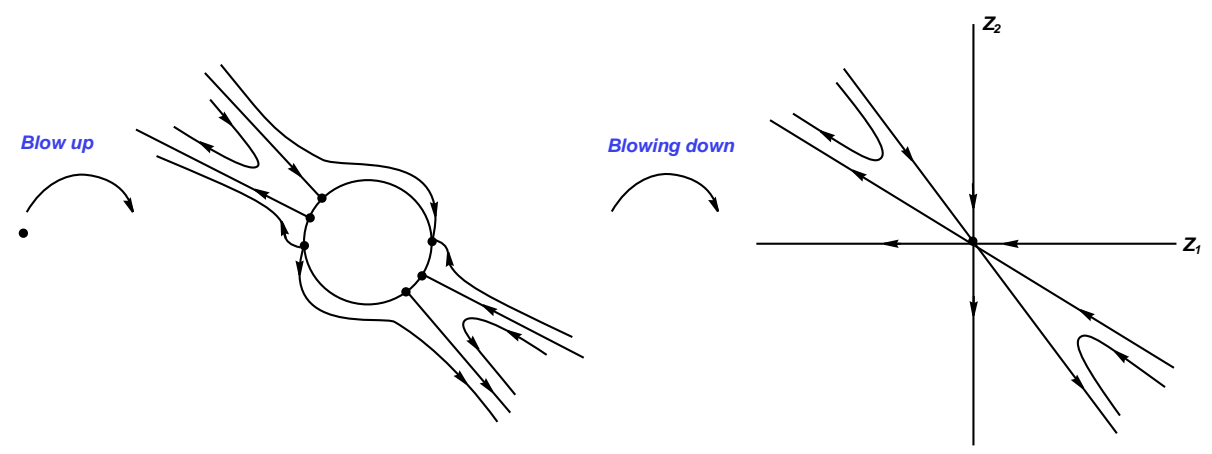

Figura 2.21: $b_{00}>0$ e $b_{10}>0$.

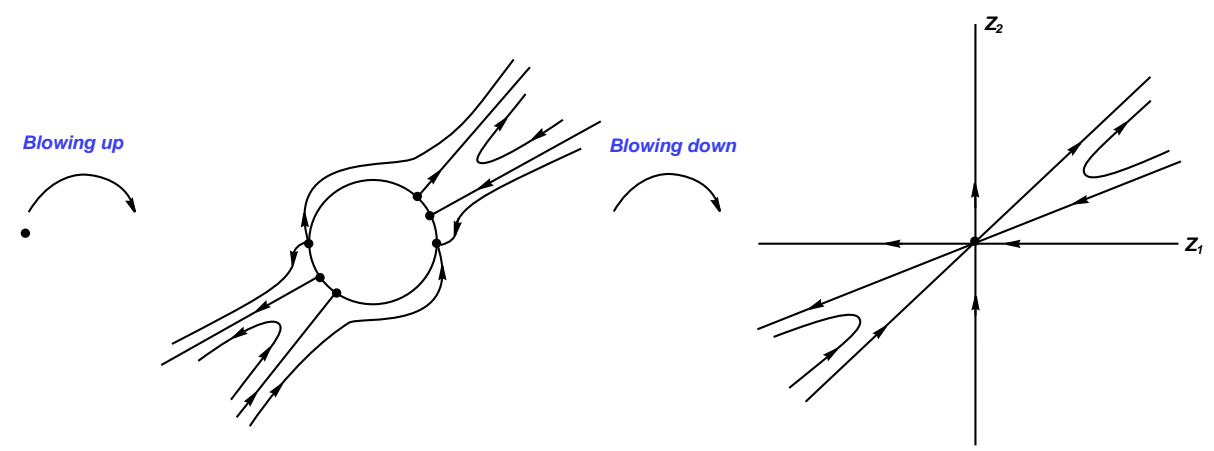

Figura 2.22: $b_{00}>0$ e $b_{10}<0$.
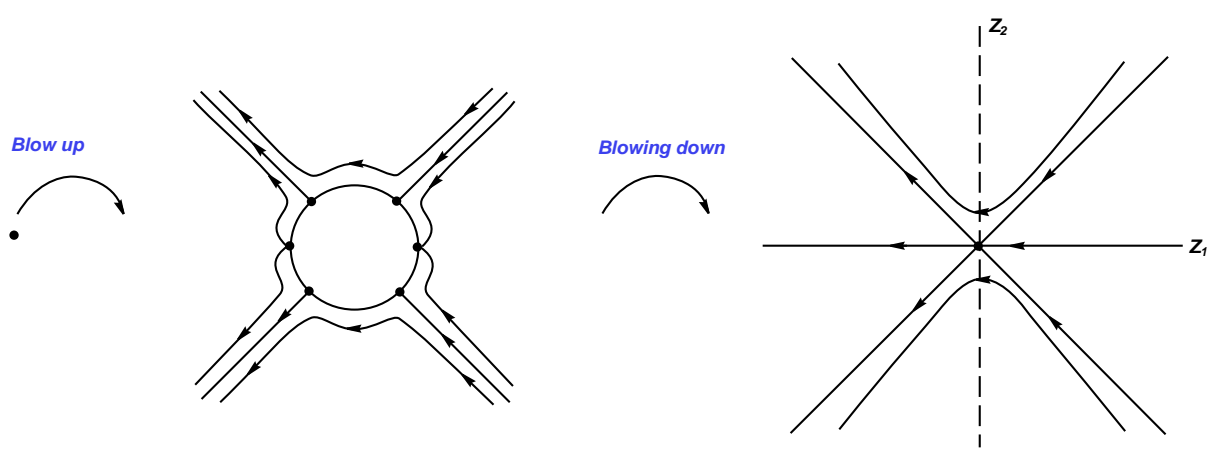

Figura 2.23: $b_{00}<0$. 
- $p>0, q>0$ e $b_{00}=0$. Como $q=b_{10}^{2}>0$ segue que $b_{10} \neq 0$.

Assim existem dois pontos singulares na direção $z_{1}$ : $(0,0)$ que, como já visto, é do tipo nó estável e $\left(0,-\frac{p}{b_{10}}\right)$ que é do tipo sela, pois

$$
J X\left(0,-\frac{p}{b_{10}}\right)=\left[\begin{array}{cc}
-p & 0 \\
0 & p
\end{array}\right] \Rightarrow \Delta=-p^{2}<0 .
$$

Como $b_{00}=0$, na direção $z_{2}$ temos $(0,0)$ singularidade do tipo sela. Fazendo um blowing down obtemos que a origem do campo vetorial (2.36) é formada por dois setores hiperbólicos separados por dois setores parabólicos como mostrado nas Figuras 2.24 e 2.25 .
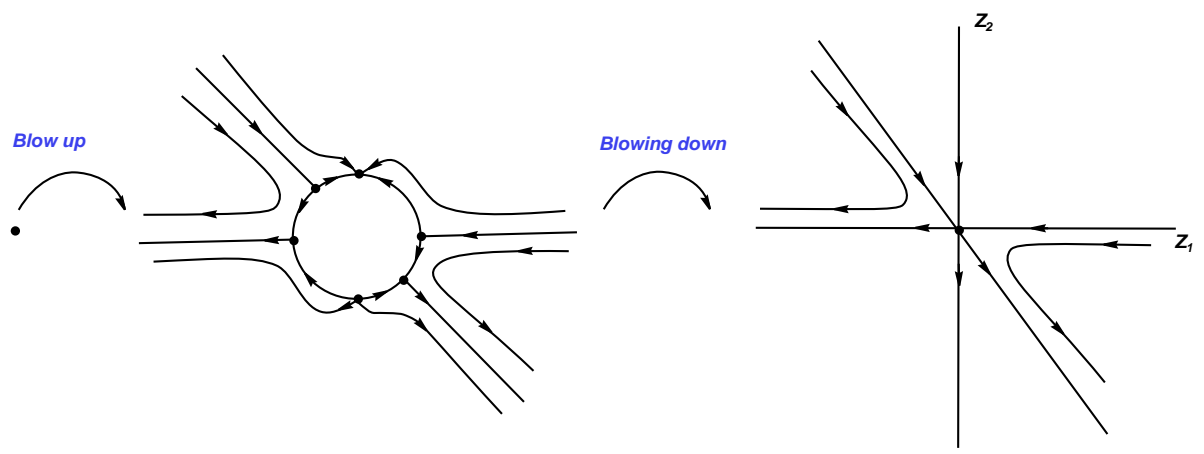

Figura 2.24: $b_{10}>0$.
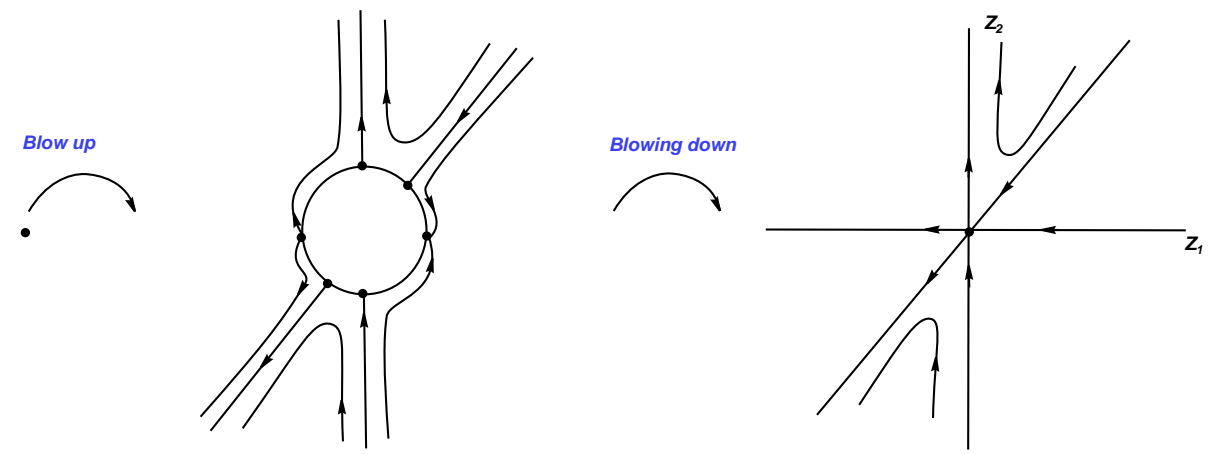

Figura 2.25: $b_{10}<0$. 
- $p>0, q<0$. O campo vetorial (2.37) tem um único ponto singular em $u=0$, o nó estável $(0,0)$. Além disso, $(0,0)$ não é singularidade na direção $z_{2}$, pois $q=$ $b_{10}^{2}-4 p b_{00}<0$ de onde segue que $b_{00} \neq 0$. Portanto, fazendo um blowing down obtemos que a origem do campo vetorial (2.36) é formada por dois setores elípticos separados por dois setores parabólicos como mostrado na Figura 2.26.
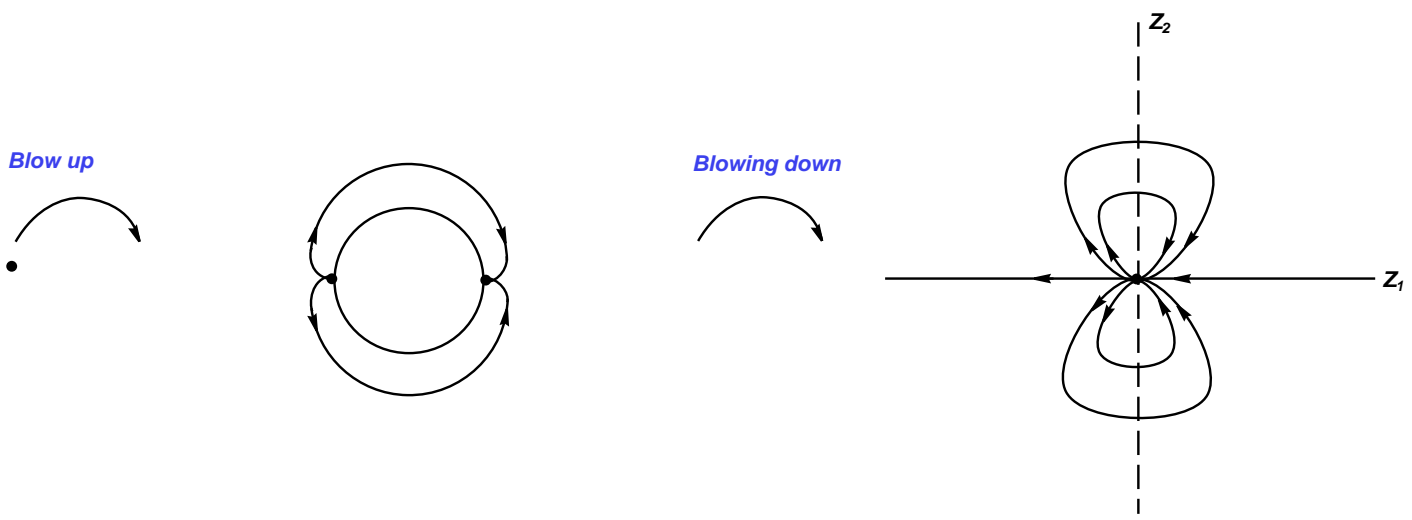

Figura 2.26: $p>0$ e $q<0$.

- $p>0, q=0$. Neste caso, temos que (2.34) é da forma:

$$
\begin{aligned}
& P=\frac{\left(2 b_{00}+b_{10} x\right)\left(2 b_{00}+b_{10} x\right)}{4 b_{00}}, \\
& Q=\frac{\left(2 b_{00}+b_{10} x\right)\left(2 b_{00} x+b_{10} y\right)}{2 b_{00}}
\end{aligned}
$$

Portanto, dividindo o sistema acima por $2 b_{00}+b_{10} x$ obtemos um campo linear.

- $p=0, q>0$ e $b_{00} \neq 0$. Em $u=0$ o campo vetorial (2.37) tem dois pontos singulares: $(0,0)$ e $\left(0,-\frac{b_{10}}{b_{00}}\right)$ sendo que o primeiro é linearmente nulo e o segundo é do tipo sela
com

$$
J X\left(0,-\frac{b_{10}}{b_{00}}\right)=\left[\begin{array}{cc}
\frac{b_{10}^{2}}{b_{00}} & 0 \\
0 & -\frac{b_{10}^{2}}{b_{00}}
\end{array}\right] \Rightarrow \Delta=-\frac{b_{10}^{4}}{b_{00}^{2}}<0
$$

Para estudar o retrato de fase numa vizinhança de $(0,0)$ façamos um segundo blow up na direção $z_{1}$. 
Aplicando a mudança de coordenadas $u=u, v=w u$ em (2.37) e dividindo por $u$ obtemos

$$
\begin{aligned}
& \dot{u}=-b_{10} u-2 b_{00} u^{2} w+b_{00} u^{2} w^{2}, \\
& \dot{w}=b_{10} w-b_{10} w^{2}+2 b_{00} u w^{2}-2 b_{00} u w^{3} .
\end{aligned}
$$

Em $u=0$ este campo vetorial tem dois pontos singulares: $(0,0)$ e $(0,1)$ com

$$
\begin{gathered}
J X(0,0)=\left[\begin{array}{cc}
-b_{10} & 0 \\
0 & b_{10}
\end{array}\right] \Rightarrow \Delta=-b_{10}^{2}<0 \quad \mathrm{e} \\
J X(0,1)=\left[\begin{array}{cc}
-b_{10} & 0 \\
0 & -b_{10}
\end{array}\right] \Rightarrow \begin{array}{l}
T=-2 b_{10} \\
T^{2}=4 \Delta=4 b_{10}^{2}
\end{array} .
\end{gathered}
$$

Então $(0,0)$ é ponto singular do tipo sela e $(0,1)$ é do tipo nó estável se $b_{10}>0$ e instável se $b_{10}<0$.

Finalmente, lembrando que na direção $z_{2},(0,0)$ não é singularidade e fazendo um blowing down primeiramente em $(u, v)=\left(z_{1} v, v\right)$ e, em seguida em $\left(z_{1}, z_{2}\right)=(u, u v)$ obtemos que a origem de (2.36) é uma singularidade do tipo sela-nó (veja Figuras $2.27,2.28,2.29$ e 2.30$)$.

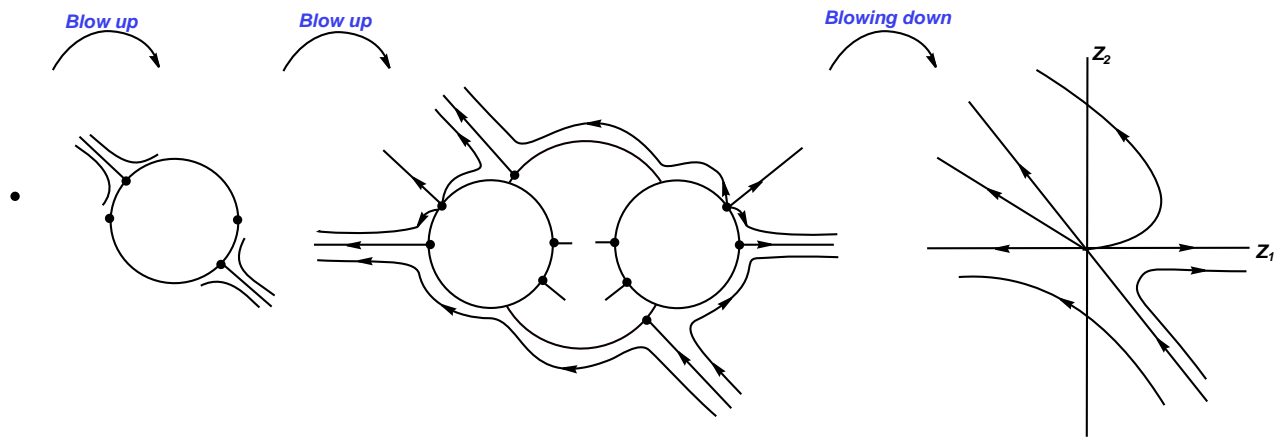

Figura 2.27: $b_{10}<0$ e $b_{00}<0$. 

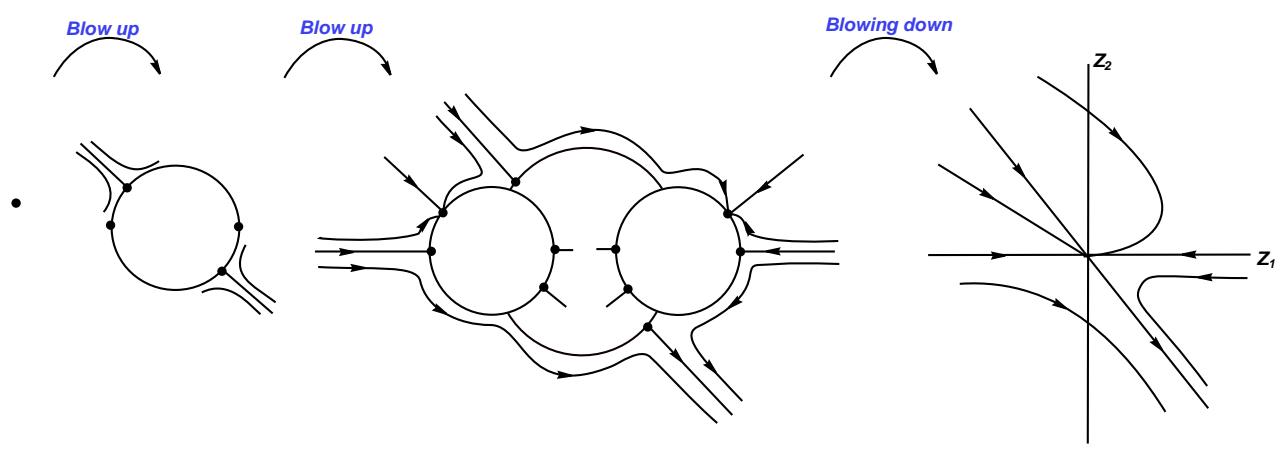

Figura 2.28: $b_{10}>0$ e $b_{00}>0$.

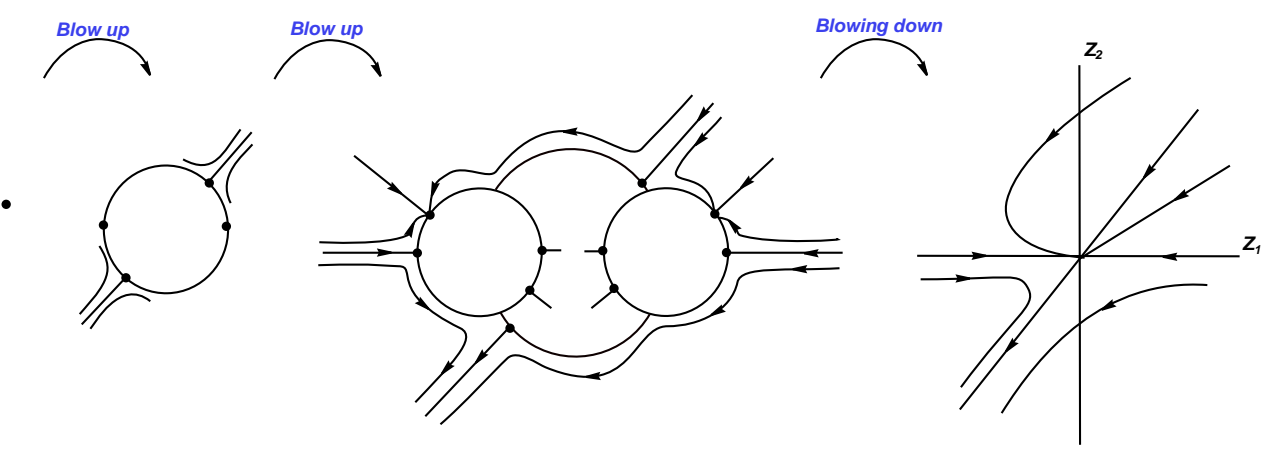

Figura 2.29: $b_{10}>0$ e $b_{00}<0$.

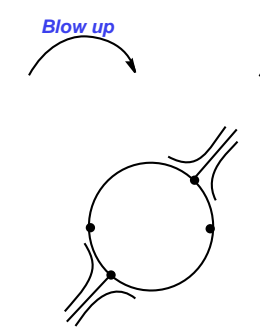

$\overbrace{}^{\text {Blow up }}$
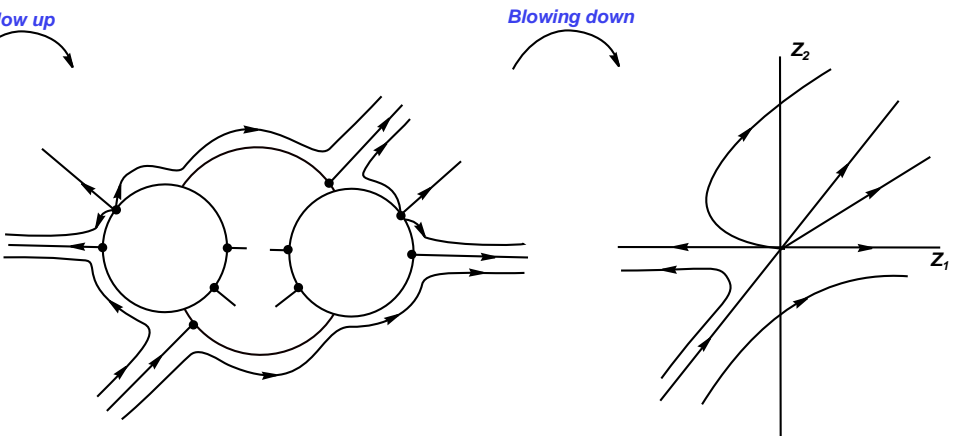

Figura 2.30: $b_{10}<0$ e $b_{00}>0$.

- $p=0, q>0$ e $b_{00}=0$. Neste caso, (0,0) é o único ponto singular de (2.37) na direção $z_{1}$ e é linearmente nulo. Além disso, como $b_{00}=0$ temos que $(0,0)$ é singularidade do tipo sela na direção $z_{2}$.

Para estudar o retrato de fase numa vizinhança de $(0,0)$ façamos um segundo blow 
$u p$ na direção $z_{1}$.

Aplicando a mudança de coordenadas $u=u, v=w u$ em (2.37) e dividindo por $u$ temos o sistema:

$$
\begin{aligned}
& \dot{u}=-b_{10} u, \\
& \dot{w}=-b_{10} w^{2}+b_{10} w .
\end{aligned}
$$

Em $u=0$ existem duas singularidades: $(0,0)$ e $(0,1)$ com:

$$
\begin{gathered}
J X(0,0)=\left[\begin{array}{cc}
-b_{10} & 0 \\
0 & b_{10}
\end{array}\right] \Rightarrow \Delta=-b_{10}^{2}<0 \quad \mathrm{e} \\
J X(0,1)=\left[\begin{array}{cc}
-b_{10} & 0 \\
0 & -b_{10}
\end{array}\right] \Rightarrow \begin{array}{l}
T=-2 b_{10} \\
T^{2}=4 \Delta=4 b_{10}^{2}
\end{array} .
\end{gathered}
$$

Logo, $(0,0)$ é do tipo sela e $(0,1)$ do tipo nó estável se $b_{10}>0$ e instável se $b_{10}<0$. Fazendo um blowing down em $(u, v)=\left(z_{1} v, v\right)$ e, depois em $\left(z_{1}, z_{2}\right)=(u, u v)$ obtemos que a origem de (2.36) é uma singularidade do tipo sela-nó (veja Figuras 2.31 e 2.32).

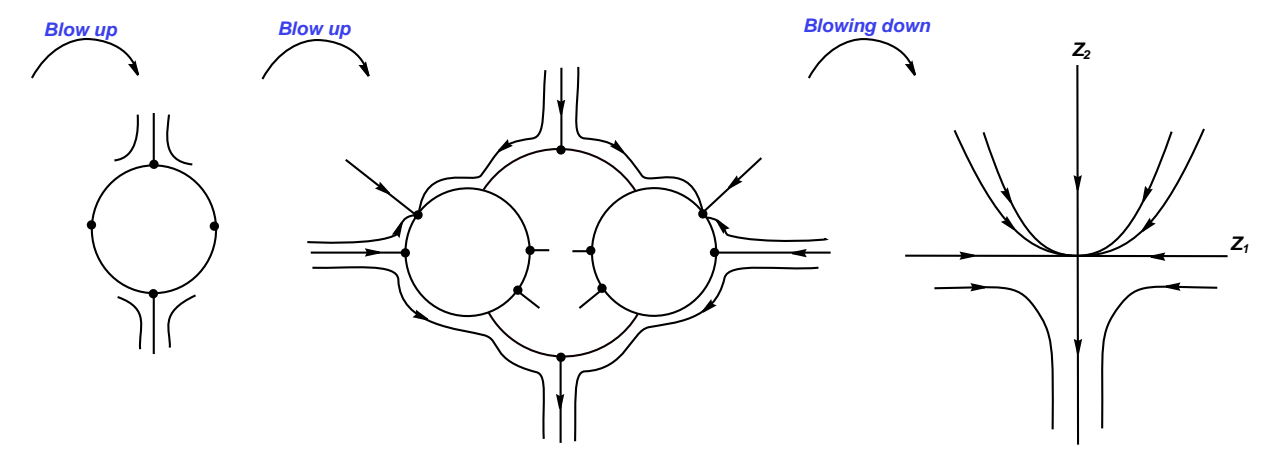

Figura 2.31: $b_{10}>0$. 

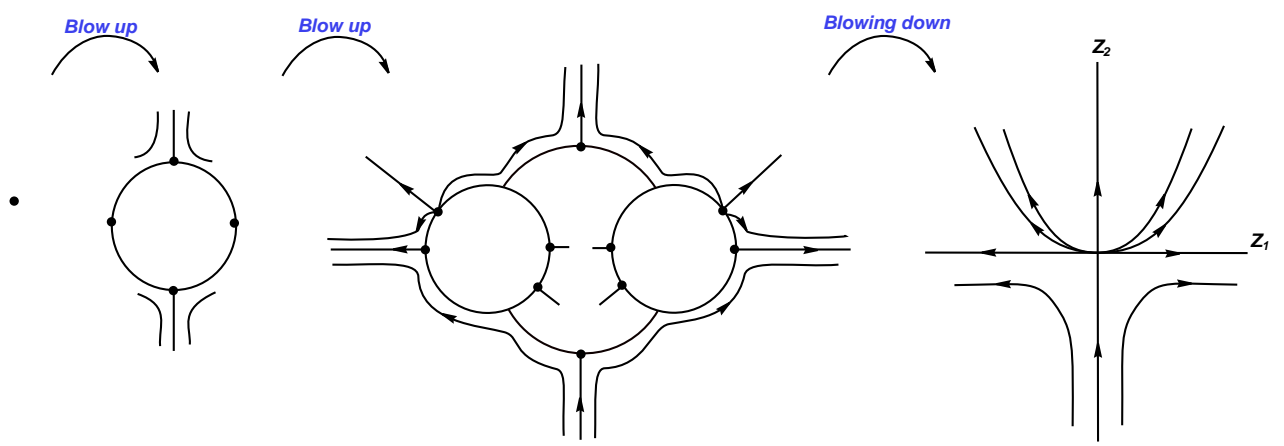

Figura 2.32: $b_{10}<0$.

- $p=0$ e $q=0$. Note que essas condições implicam que o sistema (2.34) é da forma

$$
\begin{aligned}
& P=b_{00}, \\
& Q=2 b_{00} x .
\end{aligned}
$$

isto é, (2.34) é campo linear.

Enfim, podemos desenhar os pontos singulares infinitos no disco de Poincaré (veja Figura 2.33 para o caso $p>0$ e $q>0$, Figura 2.34 para $p>0$ e $q<0$ e Figura 2.35 para $p=0$ e $q>0)$.

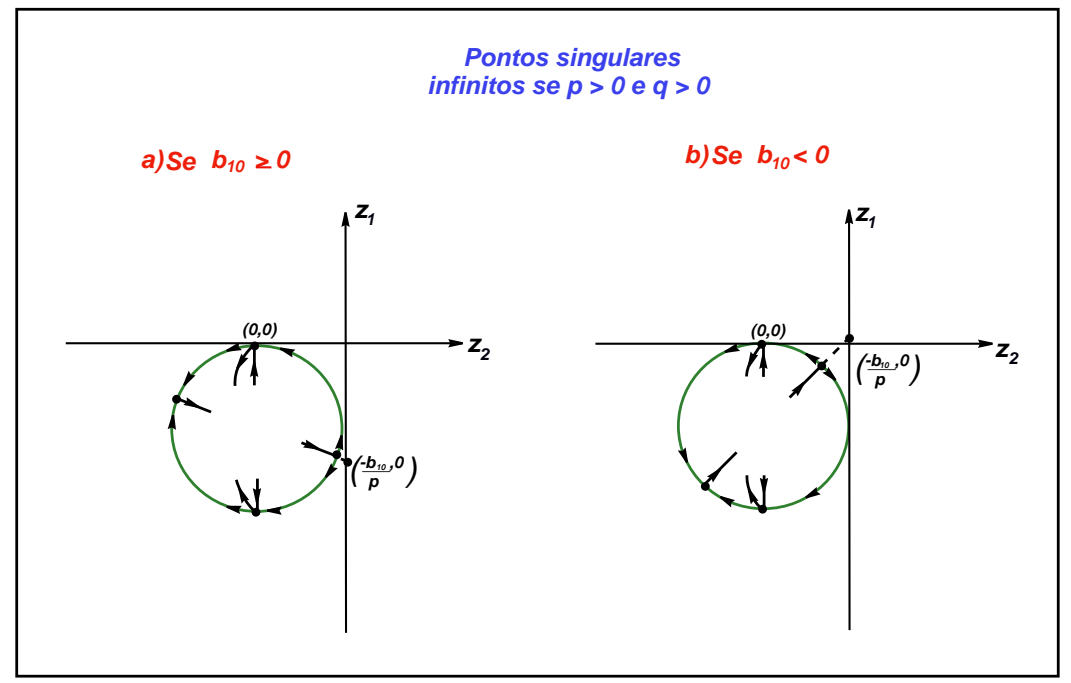

Figura 2.33: Pontos singulares infinitos para o caso $(P)$ com $p>0$ e $q>0$. 


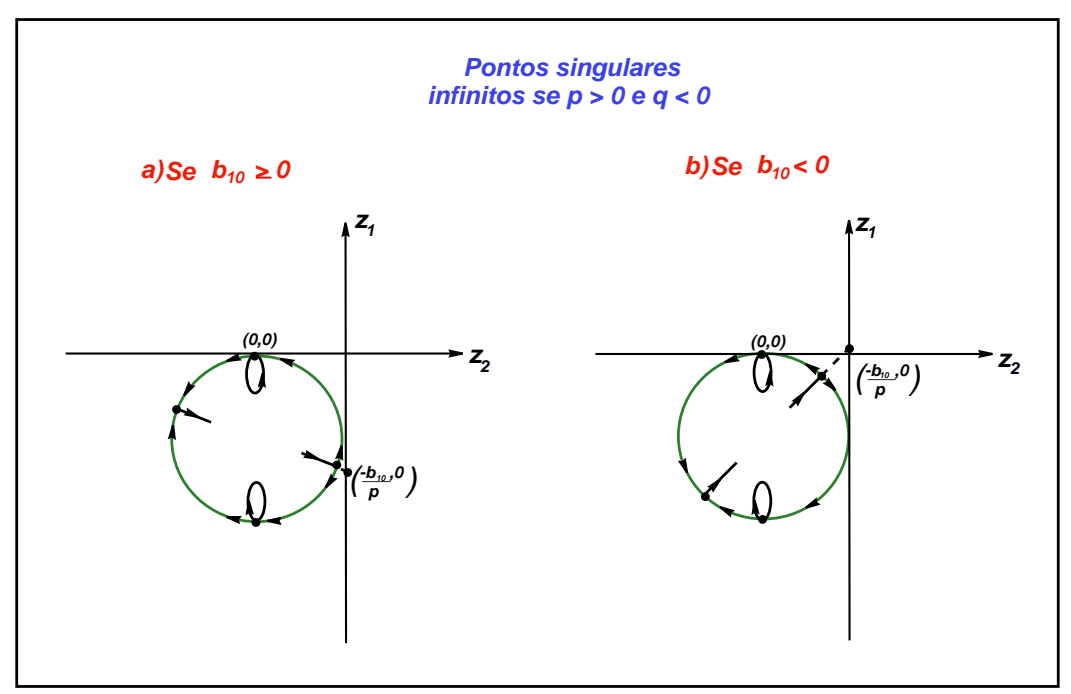

Figura 2.34: Pontos singulares infinitos para o caso $(P) \operatorname{com} p>0$ e $q<0$.

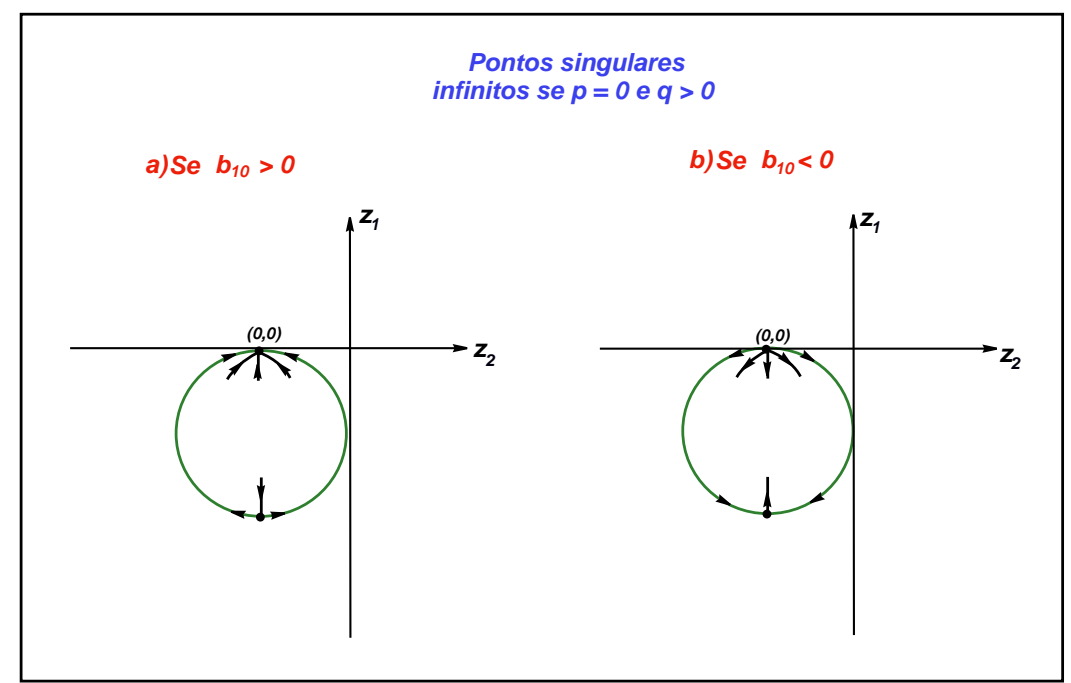

Figura 2.35: Pontos singulares infinitos para o caso $(P)$ com $p=0$ e $q>0$.

\section{Análise dos pontos singulares finitos.}

Também dividimos este estudo em casos.

- $p>0, q>0$. Existem dois pontos singulares finitos:

$$
\left(x_{1}, y_{1}\right)=\left(\frac{-b_{10}+\sqrt{q}}{2 p}, \frac{b_{10}^{2}-2 b_{00} p-b_{10} \sqrt{q}}{2 p^{2}}\right)
$$




$$
\left(x_{2}, y_{2}\right)=\left(\frac{-b_{10}-\sqrt{q}}{2 p}, \frac{b_{10}^{2}-2 b_{00} p+b_{10} \sqrt{q}}{2 p^{2}}\right)
$$

com

$$
\begin{gathered}
J X\left(x_{1}, y_{1}\right)=\left[\begin{array}{cc}
\sqrt{q} & 0 \\
0 & \sqrt{q}
\end{array}\right] \Rightarrow \begin{array}{l}
T=2 \sqrt{q} \\
T^{2}=4 \Delta=4 q
\end{array}, \\
J X\left(x_{2}, y_{2}\right)=\left[\begin{array}{cc}
-\sqrt{q} & 0 \\
0 & -\sqrt{q}
\end{array}\right] \Rightarrow \begin{array}{l}
T=-2 \sqrt{q} \\
T^{2}=4 \Delta=4 q
\end{array}
\end{gathered}
$$

Logo, são respectivamente nó instável e estável ${ }^{3}$.

- $p>0$ e $q<0$. Sob essas condições não existem pontos singulares finitos.

- $p=0$ e $q>0$. Existe um único ponto singular finito; $\left(-\frac{b_{00}}{b_{10}}, \frac{b_{00}^{2}}{b_{10}^{2}}\right)$ com

$$
J X\left(-\frac{b_{00}}{b_{10}}, \frac{b_{00}^{2}}{b_{10}^{2}}\right)=\left[\begin{array}{cc}
b_{10} & 0 \\
0 & b_{10}
\end{array}\right] \Rightarrow \begin{aligned}
& T=2 b_{10} \\
& T^{2}=4 \Delta=4 b_{10}^{2}
\end{aligned} .
$$

Consequentemente a singularidade é um nó estável se $b_{10}<0$ e instável se $b_{10}>0$.

Finalmente, reunindo as informações obtidas para os pontos singulares infinitos e finitos, podemos fazer um esboço do retrato de fase global do campo vetorial (2.34) (veja Figura 2.36 para $p<0$ e $q>0$, Figura 2.37 para $p>0$ e $q<0$, Figura 2.38 para $p=0$ e $q>0)$.

Em resumo temos:

Proposição 2.6.1. O retrato de fase de um campo vetorial polinomial quadrático planar com uma integral primeira racional de grau 2 da forma (P) dada na Proposição (2.0.7) é topologicamente equivalente aos seguintes retratos de fase da Figura 2.1:

(i) $\left(P_{1}\right)$ se $p>0$ e $q>0$;

(ii) $\left(P_{2}\right)$ se $p>0$ e $q<0$;

(iii) $\left(P_{3}\right)$ se $p=0$ e $q>0$.

\footnotetext{
${ }^{3}$ Observe que $x_{1}-x_{2}=\frac{\sqrt{q}}{2 p}>0$ o que implica que $x_{1}>x_{2}$. Além disso, $y_{1}-y_{2}=\frac{-2 b_{10} \sqrt{q}}{2 p^{2}}$, isto é, $y_{1}<y_{2}$ se $b_{10}>0$ e $y_{1}>y_{2}$ se $b_{10}<0$.
} 
Se $p>0$ e $q>0$

a) $b_{10} \geq 0$

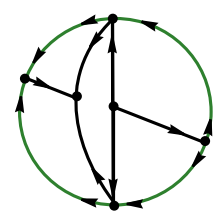

b) $b_{10}<0$

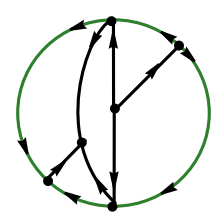

Figura 2.36: Retratos de fase no disco de Poincaré para o caso $(P)$.

Se $p>0$ e $q<0$

a) $b_{10} \geq 0$

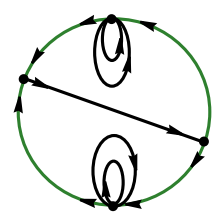

b) $b_{10}<0$

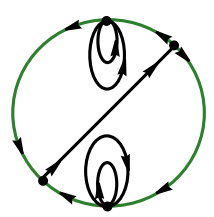

Figura 2.37: Retratos de fase no disco de Poincaré para o caso $(P)$.

$$
\text { Se } p=0 \text { e } q>0
$$

$$
\text { a) } b_{10}>0
$$

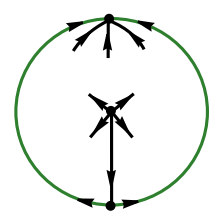

b) $b_{10}<0$

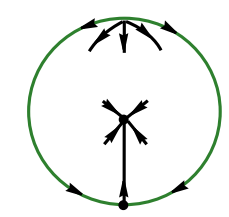

Figura 2.38: Retratos de fase no disco de Poincaré para o caso $(P)$.

\subsection{Duas retas paralelas}

Se a integral primeira racional é da forma $\frac{x^{2}-1}{D(x, y)}$, segue de $(2.1)$ que $a_{02}=a_{01}=a_{10}=$ $a_{11}=0, a_{00}=-1$ e $a_{20}=1$. Além disso, da primeira e segunda equação de (2.2) seguem, respectivamente, que $b_{11}=b_{02}=0$. O sistema cuja integral primeira tem a forma acima é: 


$$
\begin{aligned}
& P=-b_{01}+b_{01} x^{2} \\
& Q=b_{10}+2\left(b_{00}+b_{20}\right) x+b_{10} x^{2}+2 b_{01} x y .
\end{aligned}
$$

Definamos $p=b_{00}+b_{20}$. Mudando o sinal da variável independente (se necessário) podemos assumir que $b_{01} \geq 0$.

\section{Análise dos pontos singulares infinitos.}

Na carta $U_{1}$ o campo vetorial (2.40) é da forma:

$$
\begin{aligned}
& \dot{z_{1}}=b_{10}+b_{01} z_{1}+2 p z_{2}+b_{10} z_{2}^{2}+b_{01} z_{1} z_{2}^{2}, \\
& \dot{z_{2}}=-b_{01} z_{2}+b_{01} z_{2}^{3} .
\end{aligned}
$$

Se $b_{01} \neq 0,\left(-\frac{b_{10}}{b_{01}}, 0\right)$ é o único ponto singular infinito, com:

$$
J X\left(-\frac{b_{10}}{b_{01}}, 0\right)=\left[\begin{array}{cc}
b_{01} & 2 p \\
0 & -b_{01}
\end{array}\right] \Rightarrow \Delta=-b_{01}^{2}<0 .
$$

Segue que o ponto singular é uma sela.

$\mathrm{Na}$ carta $U_{2}$ temos o campo:

$$
\begin{aligned}
& \dot{z_{1}}=-b_{01} z_{1}^{2}-b_{01} z_{2}^{2}-b_{10} z_{1}^{3}-2 p z_{1}^{2} z_{2}-b_{10} z_{1} z_{2}^{2} \\
& \dot{z_{2}}=-2 b_{01} z_{1} z_{2}-b_{10} z_{1}^{2} z_{2}-2 p z_{1} z_{2}^{2}-b_{10} z_{2}^{3} .
\end{aligned}
$$

do qual $(0,0)$ é ponto singular linearmente nulo. Para estudá-lo aplicamos um blow up.

Aplicando o blow up $z_{1}=u, z_{2}=u v$ segue:

$$
\begin{aligned}
& \dot{u}=-b_{01} u^{2}-b_{10} u^{3}-2 p u^{3} v-b_{01} u^{2} v^{2}-b_{10} u^{3} v^{2} \\
& \dot{v}=-b_{01} u v+b_{01} u v^{3} .
\end{aligned}
$$

Dividindo $\dot{u}$ e $\dot{v}$ por $u$ temos:

$$
\begin{aligned}
& \dot{u}=-b_{01} u-b_{10} u^{2}-2 p u^{2} v-b_{01} u v^{2}-b_{10} u^{2} v^{2}, \\
& \dot{v}=-b_{01} v+b_{01} v^{3} .
\end{aligned}
$$

Em $u=0$ o campo vetorial acima tem três singularidades: $(0,0),(0,1)$ e $(0,-1)$ com:

$$
J X(0,0)=\left[\begin{array}{cc}
-b_{01} & 0 \\
0 & -b_{01}
\end{array}\right] \Rightarrow \begin{aligned}
& T=-2 b_{01}<0 \\
& T^{2}=4 \Delta=4 b_{01}^{2}
\end{aligned},
$$




$$
J X(0,1)=J X(0,-1)=\left[\begin{array}{cc}
-2 b_{01} & 0 \\
0 & 2 b_{01}
\end{array}\right] \Rightarrow \Delta=-4 b_{01}^{2}<0
$$

Logo, $(0,0)$ é ponto singular do tipo nó estável e, $(0,1)$ e $(0,-1)$ são selas.

Aplicamos o blow up na $z_{1}=u v, z_{2}=v$ e encontramos:

$$
\begin{aligned}
& \dot{u}=-b_{01} u^{2} v-b_{01} v-b_{10} u^{3} v^{2}-2 p u^{2} v^{2}+b_{01} u^{2} v+b_{10} u^{3} v^{2}+2 p u^{2} v^{2}, \\
& \dot{v}=-2 b_{01} u v^{2}-b_{10} u^{2} v^{3}-2 p u v^{3}-b_{10} v^{3} .
\end{aligned}
$$

Dividindo $\dot{u}$ e $\dot{v}$ por $v$ temos:

$$
\begin{aligned}
& \dot{u}=b_{01} u^{2}-b_{01}-b_{10} u^{3} v-2 p u^{2} v+b_{10} u^{3} v+2 p u^{2} v \\
& \dot{v}=-2 b_{01} u v-b_{10} u^{2} v^{2}-2 p u v^{2}-b_{10} v^{2} .
\end{aligned}
$$

Em $v=0,(0,0)$ não é ponto singular.

Fazendo um blowing down, obtemos que a origem do campo vetorial (2.42) tem o retrato de fase local dado pela Figura 2.23.

Enfim, podemos desenhar os pontos singulares infinitos no disco de Poincaré (Veja Figura 2.39) para o caso $b_{01}>0$.

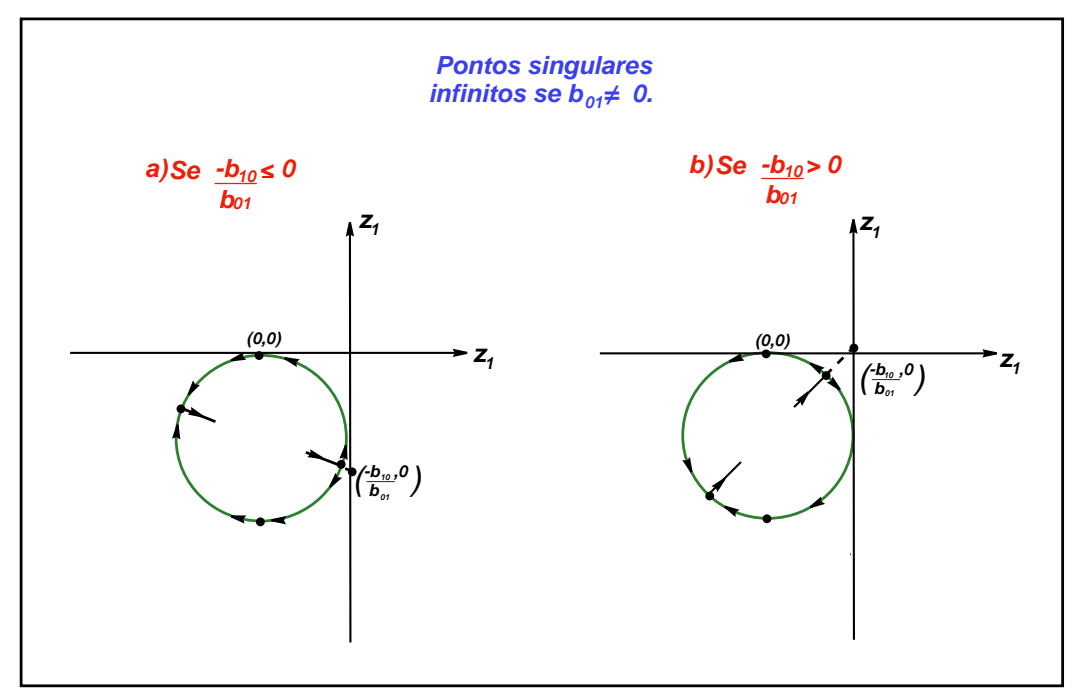

Figura 2.39: Pontos singulares infinitos para o caso $(P L)$ com $b_{01}>0$. 


\section{Análise dos pontos singulares finitos.}

Se $b_{01}>0$ os pontos singulares finitos são: $\left(x_{1}, y_{1}\right)=\left(1, \frac{-p-b_{10}}{b_{01}}\right)$ e $\left(x_{2}, y_{2}\right)=$ $\left(-1, \frac{-p+b_{10}}{b_{01}}\right) \mathrm{com}:$

$$
\begin{gathered}
J X\left(1, \frac{-p-b_{10}}{b_{01}}\right)=\left[\begin{array}{cc}
2 b_{01} & 0 \\
0 & 2 b_{01}
\end{array}\right] \Rightarrow \begin{array}{l}
T=4 b_{01}>0 \\
T^{2}=4 \Delta=16 b_{01}^{2}
\end{array}, \\
J X\left(-1, \frac{-p+b_{10}}{b_{01}}\right)=\left[\begin{array}{cc}
-2 b_{01} & 0 \\
0 & -2 b_{01}
\end{array}\right] \Rightarrow \begin{array}{l}
T=-4 b_{01}<0 \\
T^{2}=4 \Delta=16 b_{01}^{2}
\end{array} .
\end{gathered}
$$

Logo, são singularidades do tipo nó instável e estável, respectivamente.

Por propósitos práticos, façamos uma análise de como estas singularidades estão dispostas no plano.

Suponha $b_{10} \geq 0$ então $y_{1}=\frac{-p-b_{10}}{b_{01}} \leq 0$. Além disso, note que $y_{1}-y_{2}=-\frac{2 b_{10}}{b_{01}}<0$ o que implica em $y_{1}<y_{2}$ (veja Figura 2.40).

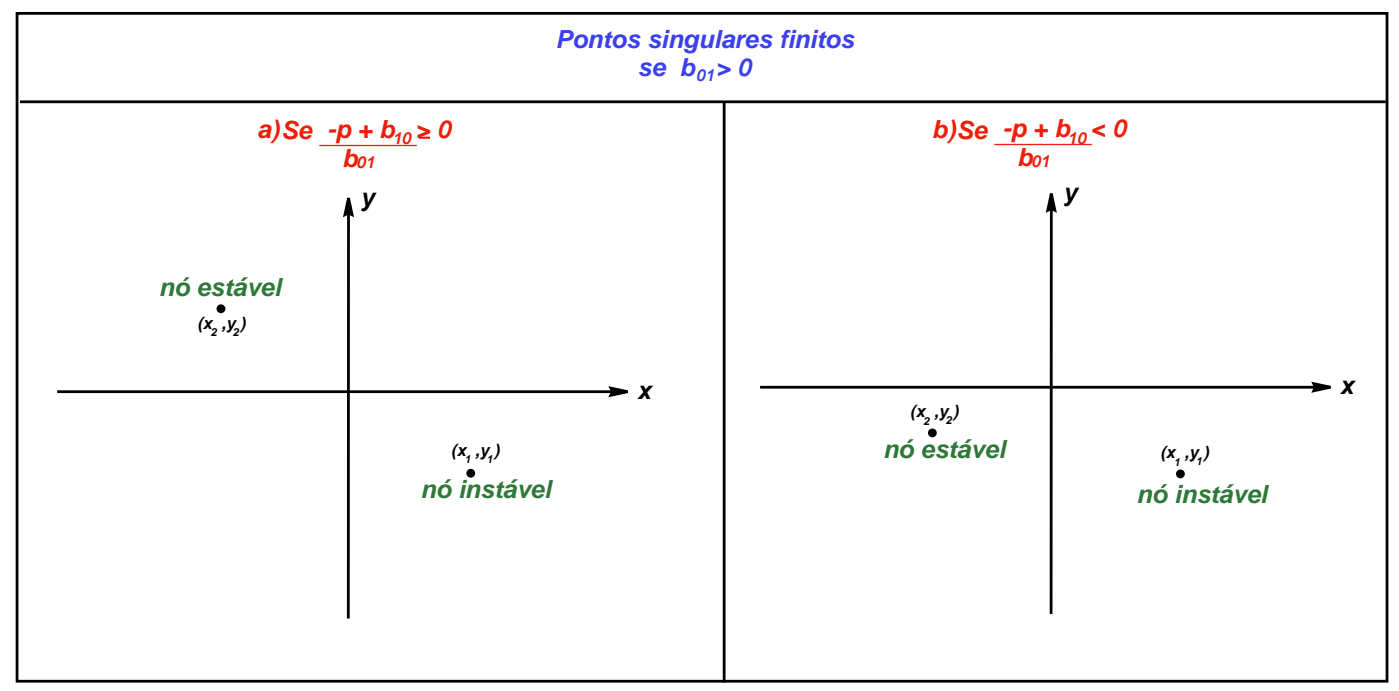

Figura 2.40: Pontos singulares finitos para o caso $(P L)$ com $b_{01}>0$.

Agora se $b_{10}<0$ temos $y_{2}=\frac{-p+b_{10}}{b_{01}}<0$ e $y_{1}-y_{2}>0$ e daí $y_{1}<y_{2}<0$ (veja Figura $2.40 \mathrm{~b}))$.

Finalmente, reunindo as informações obtidas para os pontos singulares infinitos e finitos, podemos fazer um esboço do retrato de fase global do campo vetorial (2.40) para o caso $b_{01} \neq 0$. Observe Figura 2.41. 


$$
\text { a) Se } \frac{-b_{10}}{b_{01}} \leq 0
$$

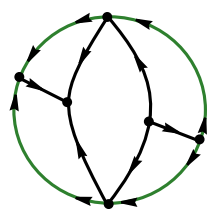

b) Se $\frac{-b_{10}}{b_{01}}>0$

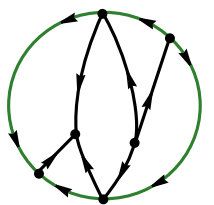

Figura 2.41: Retratos de fase no disco de Poincaré para o caso $(P L)$ com $b_{01} \neq 0$.

Note, na Figura 2.41 que os retratos a) e b) são topologicamente equivalentes.

Agora estudamos o caso $b_{01}=0$.

Neste caso, o campo vetorial (2.40) fica:

$$
\begin{aligned}
& \dot{x}=P=0, \\
& \dot{y}=Q=b_{10}+2\left(b_{00}+b_{20}\right) x+b_{10} x^{2} .
\end{aligned}
$$

Dividindo $P$ e $Q$ por $b_{10}+2\left(b_{00}+b_{20}\right) x+b_{10} x^{2}$, obtemos o campo:

$$
\begin{aligned}
& \dot{x}=P=0, \\
& \dot{y}=Q=1 .
\end{aligned}
$$

Portanto, não consideraremos este caso.

Em resumo temos:

Proposição 2.7.1. O retrato de fase de um campo vetorial polinomial quadrático planar com uma integral primeira racional de grau 2 da forma (PL) dada na Proposição (2.0.7) é topologicamente equivalente ao seguinte retrato de fase da Figura 2.1:

(i) $\left(P_{1}\right)$ se $b_{01} \neq 0$.

\subsection{Duas retas complexas paralelas}

Se a integral primeira racional é da forma $\frac{x^{2}+1}{D(x, y)}$, segue de $(2.1)$ que $a_{02}=a_{01}=$ $a_{10}=a_{11}=0, a_{00}=a_{20}=1$. Além disso, da primeira e segunda equação de (2.2) seguem, respectivamente, que $b_{11}=b_{02}=0$. Então o sistema cuja integral primeira tem a forma acima é: 


$$
\begin{aligned}
& P=b_{01}+b_{01} x^{2}, \\
& Q=-b_{10}+2\left(b_{00}-b_{20}\right) x+b_{10} x^{2}+2 b_{01} x y .
\end{aligned}
$$

Definamos $p=b_{00}-b_{20}$. Mudando o sinal da variável independente (se necessário) podemos assumir que $b_{01} \geq 0$.

\section{Análise dos pontos singulares infinitos.}

Na carta $U_{1}$ o campo vetorial (2.40) é da forma:

$$
\begin{aligned}
& \dot{z}_{1}=b_{10}+b_{01} z_{1}+2 p z_{2}-b_{10} z_{2}^{2}-b_{01} z_{1} z_{2}^{2} \\
& \dot{z}_{2}=-b_{01} z_{2}-b_{01} z_{2}^{3} .
\end{aligned}
$$

Se $b_{01} \neq 0$ existe um único ponto singular infinito: $\left(-\frac{b_{10}}{b_{01}}, 0\right)$, com:

$$
J X\left(-\frac{b_{10}}{b_{01}}, 0\right)=\left[\begin{array}{cc}
b_{01} & 2 p \\
0 & -b_{01}
\end{array}\right] \Rightarrow \Delta=-b_{01}^{2}<0
$$

e segue que o ponto singular é uma sela.

Na carta $U_{2}$ temos o campo:

$$
\begin{aligned}
& \dot{z}_{1}=-b_{01} z_{1}^{2}+b_{01} z_{2}^{2}-b_{10} z_{1}^{3}-2 p z_{1}^{2} z_{2}+b_{10} z_{1} z_{2}^{2}, \\
& \dot{z}_{2}=-2 b_{01} z_{1} z_{2}-b_{10} z_{1}^{2} z_{2}-2 p z_{1} z_{2}^{2}+b_{10} z_{2}^{3},
\end{aligned}
$$

do qual $(0,0)$ é ponto singular linearmente nulo. Para estudá-lo aplicamos um blow-up.

Aplicando o blow up $z_{1}=u, z_{2}=u v$ segue:

$$
\begin{aligned}
& \dot{u}=-b_{01} u^{2}-b_{10} u^{3}-2 p u^{3} v+b_{01} u^{2} v^{2}+b_{10} u^{3} v^{2} \\
& \dot{v}=-b_{01} u v-b_{01} u v^{3} .
\end{aligned}
$$

Dividindo $\dot{u}$ e $\dot{v}$ por $u$ temos:

$$
\begin{aligned}
& \dot{u}=-b_{01} u-b_{10} u^{2}-2 p u^{2} v+b_{01} u v^{2}+b_{10} u^{2} v^{2}, \\
& \dot{v}=-b_{01} v+b_{01} v^{3} .
\end{aligned}
$$

Em $z_{1}=0$ o campo vetorial acima tem apenas uma singularidade na origem sendo:

$$
J X(0,0)=\left[\begin{array}{cc}
-b_{01} & 0 \\
0 & -b_{01}
\end{array}\right] \Rightarrow \begin{aligned}
& T=-2 b_{01}<0 \\
& T^{2}=4 \Delta=4 b_{01}^{2}
\end{aligned} .
$$


Logo, $(0,0)$ é ponto singular do tipo nó estável.

Aplicamos o blow up $z_{1}=u v, z_{2}=v$ e encontramos:

$$
\begin{aligned}
& \dot{u}=b_{01} u^{2} v+b_{01} v-b_{10} u^{3} v^{2}+b_{10} u^{3} v^{2}, \\
& \dot{v}=-2 b_{01} u v^{2}-b_{10} u^{2} v^{3}-2 p u v^{3}+b_{10} v^{3} .
\end{aligned}
$$

Dividindo $\dot{u}$ e $\dot{v}$ por $v$ temos:

$$
\begin{aligned}
& \dot{u}=b_{01} u^{2}+b_{01}-b_{10} u^{3} v+b_{10} u^{3} v, \\
& \dot{v}=-2 b_{01} u v-b_{10} u^{2} v^{2}-2 p u v^{2}+b_{10} v^{2} .
\end{aligned}
$$

Em $v=0$, não existem pontos singulares.

Fazendo um blowing down, concluímos que, a origem do campo vetorial (2.46) tem o retrato de fase local formado por dois setores elipticos separados por dois setores parabólicos (veja Figura 2.26).

Assim, a disposição dos pontos singulares infinitos no disco de Poincaré é análoga a do caso (PL) para $b_{01}>0$ (Veja Figura 2.39).

Note que se $b_{01} \neq 0$ então não existem pontos singulares finitos. Logo, o retrato de fase global do campo vetorial (2.42) no disco de Poincaré é o ilustrado na Figura 2.42.

a) Se $\frac{-b_{10}}{b_{01}} \leq 0$

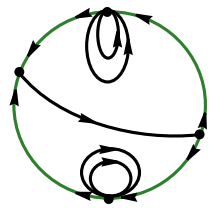

b) Se $\frac{-b_{10}}{b_{01}}>0$

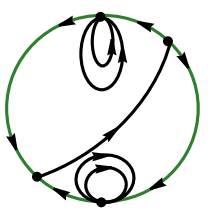

Figura 2.42: Retratos de fase no disco de Poincaré para o caso $(C L)$ com $b_{01} \neq 0$.

A seguir estudamos o caso $b_{01}=0$. Notamos que neste caso, (2.44) é da forma:

$$
\begin{aligned}
& P=0, \\
& Q=-b_{10}+2\left(b_{00}-b_{20}\right) x+b_{10} x^{2} .
\end{aligned}
$$

Então dividindo $P$ e $Q$ por $-b_{10}+2\left(b_{00}-b_{20}\right) x+b_{10} x^{2}$, obtemos

$$
\begin{aligned}
& P=0, \\
& Q=1 .
\end{aligned}
$$


Portanto, não consideraremos este caso.

Resumidamente, temos:

Proposição 2.8.1. O retrato de fase de um campo vetorial polinomial quadrático planar com uma integral primeira racional de grau 2 da forma (CL) dada na Proposição (2.0.7) é topologicamente equivalente ao seguinte retrato de fase da Figura 2.1:

(i) $\left(P_{2}\right)$ se $b_{01} \neq 0$.

\subsection{Uma reta dupla}

Se a integral primeira racional é da forma $\frac{x^{2}}{D(x, y)}$, segue de $(2.1)$ que $a_{00}=a_{02}=$ $a_{01}=a_{10}=a_{11}=0, a_{20}=1$. Além disso, da primeira e segunda equação de (2.2) seguem, respectivamente, que $b_{11}=b_{02}=0$. Temos então o sistema:

$$
\begin{aligned}
& P=b_{01} x^{2}, \\
& Q=2 b_{00} x+b_{10} x^{2}+2 b_{01} x y .
\end{aligned}
$$

Note que neste caso, $P$ e $Q$ têm $x$ como fator comum. Logo, dividindo (2.49) por $x$ obtemos um campo linear.

\subsection{Infinito degenerado}

Para completar o estudo de todos os retratos de fase dos campos vetoriais quadráticos com integral primeira racional de grau 2, pelo Lema 2.0.6, precisamos estudar os sistemas da forma:

$$
\begin{aligned}
& P=2 b_{00}+2 b_{10} x+b_{01} y+2 b_{20} x^{2}+b_{11} x y, \\
& Q=y\left(b_{10}+2 b_{20} x+b_{11} x y\right) .
\end{aligned}
$$

Definamos $q=b_{10}^{2}-4 b_{00} b_{20}$ e $r=b_{10} b_{11}-2 b_{01} b_{20}$. Observamos que $b_{20}=b_{11}=0$ não pode ocorrer, caso contrário, o sistema (2.50) é linear.

\section{Análise dos pontos singulares infinitos:}

Na carta $U_{1}$ o campo vetorial (2.50) é da forma: 


$$
\begin{aligned}
& \dot{z_{1}}=-z_{1} z_{2}\left(b_{10}+b_{01} z_{1}+2 b_{00} z_{2}\right) \\
& \dot{z}_{2}=-z_{2}\left(2 b_{20}+b_{11} z_{1}+2 b_{10} z_{2}+b_{01} z_{1} z_{2}+2 b_{00} z_{2}^{2}\right) .
\end{aligned}
$$

Então se $z_{2}=0$ decorre que $\dot{z}_{1}=\dot{z}_{2}=0$. Logo, todos os pontos no infinito são singulares.

Dividindo (2.51) por $z_{2}$ obtemos:

$$
\begin{aligned}
& \dot{z}_{1}=-z_{1}\left(b_{10}+b_{01} z_{1}+2 b_{00} z_{2}\right), \\
& \dot{z}_{2}=-2 b_{20}-b_{11} z_{1}-2 b_{10} z_{2}-b_{01} z_{1} z_{2}-2 b_{00} z_{2}^{2} .
\end{aligned}
$$

Assim, em $z_{2}=0$ o campo (2.52) tem a singularidade $\left(-\frac{b_{10}}{b_{01}}, 0\right)$ se, e somente se, $r=0$. E tem a singularidade $(0,0)$ se, e somente se, $b_{20}=0$.

Em $U_{2}$ o campo vetorial (2.50) é da forma:

$$
\begin{aligned}
& \dot{z_{1}}=z_{2}\left(b_{01}+b_{10} z_{1}+2 b_{00} z_{2}\right), \\
& \dot{z_{2}}=-z_{2}\left(b_{11} z_{1}+2 b_{20} z_{1}+b_{10} z_{2}\right) .
\end{aligned}
$$

Dividindo o sistema acima por $z_{2}$ :

$$
\begin{aligned}
& \dot{z_{1}}=b_{10}+b_{01} z_{1}+2 b_{00} z_{2}, \\
& \dot{z_{2}}=b_{11} z_{1}+2 b_{20} z_{1}+b_{10} z_{2} .
\end{aligned}
$$

É imediato que se $b_{10} \neq 0,(0,0)$ não é singularidade de $(2.54)$.

\section{Análise dos pontos singulares finitos.}

Dividimos este estudo em nove casos:

i) $q>0$ e $b_{20} r \neq 0$. Temos três pontos singulares:

$$
P_{1}=\left(\frac{-b_{10}-\sqrt{q}}{2 b_{20}}, 0\right), P_{2}=\left(\frac{-b_{10}+\sqrt{q}}{2 b_{20}}, 0\right), P_{3}=\left(\frac{b_{01} b_{10}-2 b_{00} b_{11}}{r},-\frac{q}{r}\right)
$$

com

$$
J X\left(P_{1}\right)=\left[\begin{array}{cc}
-2 \sqrt{q} & \frac{-r+b_{11} \sqrt{q}}{2 b_{20}} \\
0 & -\sqrt{q}
\end{array}\right] \Rightarrow \begin{aligned}
& T=-3 \sqrt{q}<0 \\
& T^{2}=9 q>4 \Delta=8 q
\end{aligned}
$$




$$
\begin{gathered}
\left.J X\left(P_{2}\right)=\left[\begin{array}{cc}
2 \sqrt{q} & \frac{-r+b_{11} \sqrt{q}}{2 b_{20}} \\
0 & \sqrt{q}
\end{array}\right] \Rightarrow \begin{array}{l}
T=3 \sqrt{q}>0 \\
T^{2}=9 q>4 \Delta=8 q \\
J X\left(P_{3}\right)=\left[\begin{array}{cc}
\frac{b_{11} q}{r} & -\frac{2 b_{01}\left(-b_{10} b_{11}+b_{01} b_{20}\right)+2 b_{00} b_{10}^{2}}{r} \\
-\frac{2 b_{20} q}{r} & -\frac{b_{11} q}{r}
\end{array}\right] \Rightarrow \Delta=-q<0
\end{array}\right]
\end{gathered}
$$

Logo, $P_{1}, P_{2}$ são respectivamente nó estável e instável e $P_{3}$ é singularidade do tipo sela. Juntando a isto o fato que todos os pontos no infinito são singulares segue que o retrato de fase do campo (2.50) neste caso é dado pela Figura 2.43.

Se $b_{20} r \neq 0$ e $q>0$

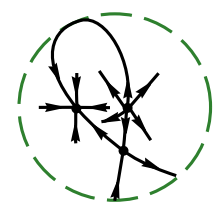

Figura 2.43: Retrato de fase no disco de Poincaré para o caso $(D I)$.

ii) $q=0$ e $b_{20} r \neq 0$. Neste caso, o único ponto singular finito é $\left(-\frac{b_{10}}{2 b_{20}}, 0\right)$ com:

$$
J X\left(-\frac{b_{10}}{2 b_{20}}, 0\right)=\left[\begin{array}{cc}
0 & -\frac{r}{2 b_{20}} \\
0 & 0
\end{array}\right] .
$$

Assim, $\left(-\frac{b_{10}}{2 b_{20}}, 0\right)$ é ponto singular nilpotente. Para estudá-lo aplicamos a Proposição (1.2.2). Primeiramente, transladamos o ponto singular para a origem. Para isso, aplicamos a seguinte mudança de coordenadas: $X=x+\frac{b_{10}}{2 b_{20}}, Y=y$, ao sistema (2.50). Obtemos: 


$$
\begin{aligned}
& P=Y-\frac{b_{10}^{4}}{4 r b_{00}^{2}} X^{2}-\frac{b_{11} b_{10}^{2}}{2 r b_{00}} X Y, \\
& Q=-\frac{b_{10}^{4}}{4 r b_{00}^{2}} X Y-\frac{b_{11} b_{10}^{2}}{2 r b_{00}} Y^{2}
\end{aligned}
$$

que é da forma: $(Y+F(X, Y), G(X, Y))$, com $F(X, Y)=-\frac{b_{10}^{4}}{4 r b_{00}^{2}} X^{2}-\frac{b_{11} b_{10}^{2}}{2 r b_{00}} X Y$, $G(X, Y)=-\frac{b_{10}^{4}}{4 r b_{00}^{2}} X Y-\frac{b_{11} b_{10}^{2}}{2 r b_{00}} Y^{2}$ e $F, G$ funções analíticas de grau 2.

Procuramos $Y$ tal que $Y=f(X)$ é solução da equação

$$
Y+F(X, Y)=0
$$

numa vizinhança de $(0,0)$.

Suponhamos que $Y=f(X)=a_{1} X+a_{2} X^{2}+a_{3} X^{3}+\ldots+a_{k} X^{k}+\ldots$ é a solução procurada. Substituindo essa expressão em (2.57) encontramos:

$a_{1} X+a_{2} X^{2}+a_{3} X^{3}+\ldots+a_{k} X^{k}+\ldots=\frac{b_{10}^{4}}{4 r b_{00}^{2}} X^{2}+\frac{b_{11} b_{10}^{2}}{2 r b_{00}}\left(a_{1} X^{2}+a_{2} X^{3}+a_{3} X^{4}+\right.$ $\left.\ldots+a_{k} X^{k+1}+\ldots\right)$.

Comparando os coeficientes $a_{i}^{\prime} s$ na equação acima obtemos:

$$
a_{1}=0 ; \quad a_{2}=\frac{b_{10}^{4}}{4 r b_{00}^{2}}+\frac{b_{11} b_{10}^{2}}{2 r b_{00}} a_{1} ; a_{3}=\frac{b_{11} b_{10}^{2}}{2 r b_{00}} a_{2}
$$

o que implica em: $a_{2}=\frac{b_{10}^{4}}{4 r b_{00}^{2}}, a_{3}=\frac{b_{11} b_{10}^{6}}{8 r^{2} b_{00}^{3}}$. Então temos

$$
\begin{aligned}
& F(X)=\frac{b_{10}^{4}}{4 r b_{00}^{2}} X^{2}+\frac{b_{11} b_{10}^{6}}{8 r^{2} b_{00}^{3}} X^{3}+\ldots, \\
& G(X, f(X)) \quad=-\frac{b_{10}^{4}}{4 r b_{00}^{2}} X\left(\frac{b_{10}^{4}}{4 r b_{00}^{2}} X^{2}+\frac{b_{11} b_{10}^{6}}{8 r^{2} b_{00}^{3}} X^{3}+\ldots\right)-\frac{b_{11} b_{10}^{2}}{2 r b_{00}}\left(\frac{b_{10}^{4}}{4 r b_{00}^{2}} X^{2}+\right.
\end{aligned}
$$

$$
\left.\frac{b_{11} b_{10}^{6}}{8 r^{2} b_{00}^{3}} X^{3}+\ldots\right)^{2}
$$

$$
=-\frac{b_{10}^{8}}{16 r^{2} b_{00}^{4}} X^{3}+O\left(x^{3}\right)=K x^{\kappa}+O\left(x^{\kappa}\right)
$$

$\left(\frac{\partial F}{\partial X}+\frac{\partial G}{\partial Y}\right)(X, f(X))=-\frac{3 b_{10}^{4}}{4 r b_{00}^{2}} X+O(x)=L x^{\lambda}+O\left(x^{\lambda}\right)$ 
Segue que $K=-\frac{b_{10}^{8}}{16 r^{2} b_{00}^{4}}, \kappa=3, L=-\frac{3 b_{10}^{4}}{4 r b_{00}^{2}}, \lambda=1$. Logo, $\kappa=2 \lambda+1$ e $L^{2}+$ $4 K(\lambda+1)=\frac{b_{10}^{8}}{16 r^{2} b_{00}^{4}} \geq 0$. Portanto, pelo item (3.b) da Proposição 1.2.2 concluímos que o ponto singular $\left(-\frac{b_{10}}{2 b_{20}}, 0\right)$ é formado pela união de um setor hiperbólico e um setor elíptico.

Lembrando que todo o infinito é singularidade, temos que o retrato de fase global do campo (2.50) é dado pela Figura 2.44.

Se $b_{20} r \neq 0$ e $q=0$

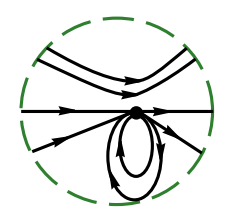

Figura 2.44: Retrato de fase no disco de Poincaré para o caso $(D I)$.

iii) $q<0$ e $b_{20} r \neq 0$, isto é, o único ponto singular é $P_{3}$. Observamos que de (2.55) segue que $T=0$ e $\Delta=-q>0$. Logo, $P_{3}$ é ponto singular do tipo centro. E, o retrato de fase do campo vetorial (2.50) neste caso, é o dado pela Figura 2.45.

Se $b_{20} r \neq 0$ e $q<0$

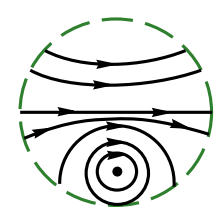

Figura 2.45: Retrato de fase no disco de Poincaré para o caso $(D I)$.

iv) $q>0$ e $b_{20} \neq 0$ e $r=0$, isto é, existem dois pontos singulares: $P_{1}$ e $P_{2}$ e, como visto no item $i$ ) são respectivamente nó estável e instável.

É importante notarmos que todos os pontos infinitos são singulares, porém, neste caso, $\left(-\frac{b_{10}}{b_{01}}, 0\right)$ é um ponto singular que se destaca em relação aos demais. Sua matriz Jacobiana é dada por: 


$$
J X\left(-\frac{b_{10}}{b_{01}}, 0\right)=\left[\begin{array}{cc}
b_{10} & \frac{2 b_{00} b_{10}}{b_{01}} \\
-b_{11} & -b_{10}
\end{array}\right] \Rightarrow \Delta=-q<0
$$

isto é, $\left(-\frac{b_{10}}{b_{01}}, 0\right)$ é ponto singular infinito do tipo sela.

Portanto, o retrato de fase de (2.50) é o dado pela Figura 2.46.

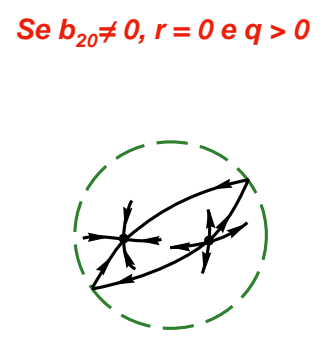

Figura 2.46: Retrato de fase no disco de Poincaré para o caso $(D I)$.

v) $q=0$ e $b_{20} \neq 0$ e $r=0$. Sob essas condições o campo vetorial (2.50) é da forma:

$$
\begin{aligned}
& P=\frac{\left(b_{10}+2 b_{20} x\right)\left(b_{10}+2 b_{20} x+b_{11} y\right)}{2 b_{20}}, \\
& Q=y\left(b_{10}+2 b_{20} x+b_{11} x y\right) .
\end{aligned}
$$

Dividindo a expressão acima por $b_{10}+2 b_{20} x+b_{11} x y$ obtemos um campo linear.

vi) $q<0$ e $b_{20} \neq 0$ e $r=0$. Neste caso, não existem pontos singulares finitos.

Assim como no item iv), $\left(-\frac{b_{10}}{b_{01}}, 0\right)$ é um ponto singular infinito que se destaca em relação aos demais, porém, aqui $T=0$ e $\Delta=-q>0$. Logo, ele é do tipo centro.

Então o retrato de fase global é o dado pela Figura 2.47.

vii) $b_{20}=0$ e $r=b_{10} b_{11} \neq 0$. Aqui temos dois pontos singulares finitos: $P_{3}$ que como já vimos é do tipo sela e $\left(\frac{b_{00}}{b_{10}}, 0\right)$ com 


$$
\text { Se } b_{20} \neq 0, r=0 \text { e } q<0
$$

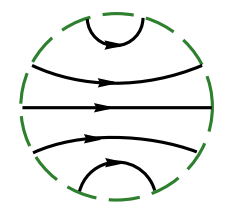

Figura 2.47: Retrato de fase no disco de Poincaré para o caso $(D I)$.

$$
J X\left(\frac{b_{00}}{b_{10}}, 0\right)=\left[\begin{array}{cc}
2 b_{10} & \frac{b_{01} b_{10}-b_{00} b_{11}}{b_{10}} \\
0 & b_{10}
\end{array}\right] \Rightarrow \begin{aligned}
& T=3 b_{10} \\
& T^{2}=9 b_{10}^{2}>4 \Delta=8 b_{10}^{2}
\end{aligned}
$$

Logo, $\left(\frac{b_{00}}{b_{10}}, 0\right)$ é do tipo nó estável se $b_{10}<0$ e instável se $b_{10}>0$.

Neste caso, $(0,0)$ é um ponto singular em $U_{1}$ que se destaca em relação aos demais.

Temos que

$$
J X(0,0)=\left[\begin{array}{cc}
-b_{10} & 0 \\
-b_{11} & -2 b_{10}
\end{array}\right] \Rightarrow \begin{aligned}
& T=-3 b_{10} \\
& T^{2}=9 b_{10}^{2}>4 \Delta=8 b_{10}^{2}
\end{aligned}
$$

isto é, a origem da carta $U_{1}$ é um ponto singular do tipo nó estável se $b_{10}>0$ e do tipo nó instável se $b_{10}<0$.

Concluímos portanto, que o retrato de fase global de (2.50), nessas condições, é o dado na Figura 2.48.

$$
\text { Se } b_{20}=0, r=b_{10} b_{11} \neq 0
$$

$$
\text { a) } \operatorname{Se} b_{10}>0
$$

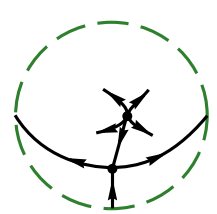

b) $\operatorname{Se} b_{10}<0$

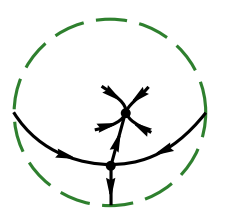

Figura 2.48: Retratos de fase no disco de Poincaré para o caso $(D I)$. 
viii) $b_{20}=b_{10}=0$ e $b_{00} b_{11} \neq 0$. Neste caso, não existem pontos singulares finitos.

Como no caso anterior, $(0,0)$ é ponto singular infinito em $U_{1}$ que se destaca dos demais. Porém aqui, ele é um ponto singular nilpotente (pois $b_{10}=0$ ). Para estudar seu retrato de fase devemos aplicar a Proposição 1.2.2.

Primeiramente, devemos colocar o sistema (2.52) na forma $(Y+F(X, Y), G(X, Y))$ com $F$ e $G$ satisfazendo as condições da Proposição 1.2.2. Para isto, dividimos (2.52) por $-b_{11}$ e, em seguida, fazemos a mudança de variáveis: $z_{1}=Y, z_{2}=X$. Obtemos

$$
\begin{aligned}
& \dot{X}=Y+\frac{b_{01}}{b_{11}} X Y+\frac{2 b_{00}}{b_{11}} X^{2}, \\
& \dot{Y}=\frac{b_{01}}{b_{11}} Y^{2}+\frac{2 b_{00}}{b_{11}} X Y,
\end{aligned}
$$

que está na forma desejada.

Procuramos $Y$ tal que $Y=f(X)$ é solução da equação

$$
Y+F(X, Y)=0
$$

numa vizinhança de $(0,0)$, onde $F(X, Y)=\frac{b_{01}}{b_{11}} X Y+\frac{2 b_{00}}{b_{11}} X^{2}$ e $G(X, Y)=\frac{b_{01}}{b_{11}} Y^{2}+$ $\frac{2 b_{00}}{b_{11}} X Y$.

Suponhamos que $Y=f(X)=a_{1} X+a_{2} X^{2}+a_{3} X^{3}+\ldots+a_{k} X^{k}+\ldots$ é a solução procurada.

Substituindo essa expressão em (2.58) encontramos:

$a_{1} X+a_{2} X^{2}+a_{3} X^{3}+\ldots+a_{k} X^{k}+\ldots=-\frac{2 b_{00}}{b_{11}} X^{2}-\frac{b_{01}}{b_{11}}\left(a_{1} X^{2}+a_{2} X^{3}+a_{3} X^{4}+\ldots+\right.$ $\left.a_{k} X^{k+1}+\ldots\right)$

Comparando os coeficientes $a_{i}^{\prime} s$ na equação acima obtemos:

$$
a_{1}=0 ; \quad a_{2}=-\frac{2 b_{00}}{b_{11}}-\frac{b_{01}}{b_{11}} a_{1} ; a_{3}=-\frac{b_{01}}{b_{11}} a_{2}
$$

o que implica em: $a_{2}=-\frac{2 b_{00}}{b_{11}}, a_{3}=\frac{2 b_{01} b_{00}}{b_{11}^{2}}$. Então temos 


$$
\begin{aligned}
& F(X)=\frac{-2 b_{00}}{b_{11}} X^{2}+\frac{2 b_{01} b_{00}}{b_{11}^{2}} X^{3}+\ldots \\
& G(X, f(X))=-\frac{4 b_{00}^{2}}{b_{11^{2}}} X^{3}+O\left(x^{3}\right)=K x^{\kappa}+O\left(x^{\kappa}\right) \\
& \left(\frac{\partial F}{\partial X}+\frac{\partial G}{\partial Y}\right)(X, f(X))=\frac{6 b_{00}}{b_{11}} X+O(x)=L x^{\lambda}+O\left(x^{\lambda}\right)
\end{aligned}
$$

Segue que $K=-\frac{4 b_{00}^{2}}{b_{11^{2}}}, \kappa=3, L=\frac{6 b_{00}}{b_{11}}, \lambda=1$. Logo, $\kappa=2 \lambda+1$ e $L^{2}+4 K(\lambda+1)=$ $\frac{4 b_{00}^{2}}{b_{11}^{2}} \geq 0$. Portanto, pelo item (3.b) da Proposição 1.2 .2 concluímos que $(0,0)$ é ponto singular do tipo sela elíptica, isto é, formado pela união de um setor hiperbólico e um setor elíptico.

Assim, o retrato de fase global de (2.50), nessas condições, é o dado na Figura 2.49.

$$
\text { Se } b_{20}=b_{10}=0 \text { e } r=b_{11} b_{00} \neq 0
$$

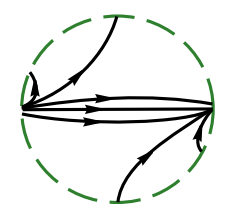

Figura 2.49: Retrato de fase no disco de Poincaré para o caso $(D I)$.

ix) $b_{20}=b_{10}=b_{00}=0$ e $b_{11} \neq 0$. Nestas condições, o campo vetorial (2.50) é da forma:

$$
\begin{aligned}
& P=b_{01} y+b_{11} x y, \\
& Q=b_{11} y^{2} .
\end{aligned}
$$

Dividindo a expressão acima por $y$ obtemos um campo linear.

Em resumo temos:

Proposição 2.10.1. O retrato de fase de um campo vetorial polinomial quadrático planar com uma integral primeira racional de grau 2, com todos os pontos do infinito singulares é topologicamente equivalente aos seguintes retratos de fase da Figura 2.1:

(i) $\left(D I_{1}\right)$ se $b_{20} r \neq 0$ e $q>0$; 
(ii) $\left(D I_{2}\right)$ se $b_{20} r \neq 0$ e $q=0$;

(iii) $\left(D I_{3}\right)$ se $b_{20} r \neq 0$ e $q<0$;

(iv) $\left(D I_{4}\right)$ se $b_{20} \neq 0, r=0$ e $q>0$;

(v) $\left(D I_{5}\right)$ se $b_{20} \neq 0, r=0$ e $q<0$;

(vi) $\left(D I_{6}\right)$ se $b_{20}=0, r=b_{10} b_{11} \neq 0$;

(vii) $\left(D I_{7}\right)$ se $b_{20}=b_{10}=0$ e $b_{11} b_{00} \neq 0$;

\subsection{Considerações Finais}

Gostaríamos de fazer algumas considerações sobre o estudo realizado.

Do estudo dos campos vetoriais quadráticos com uma integral primeira racional de grau 2, em [5], Llibre e Cairó obtiveram 25 diferentes retratos de fase globais no disco de Poincaré. Neste texto, obtivemos 18 diferentes retratos de fase no disco de Poincaré. Essa diferença se dá, porque nesse trabalho estudamos apenas os campos quadráticos da forma $X=(P, Q)$ com $P$ e $Q$ coprimos, pois os casos onde $P$ e $Q$ têm fator comum não nulo se reduzem ao estudo de campos lineares.

Gostaríamos de mencionar também, que durante nossos estudos, fizemos o uso do software P4 [3], programa computacional que estuda o retrato de fase global de campos vetoriais polinomiais. 



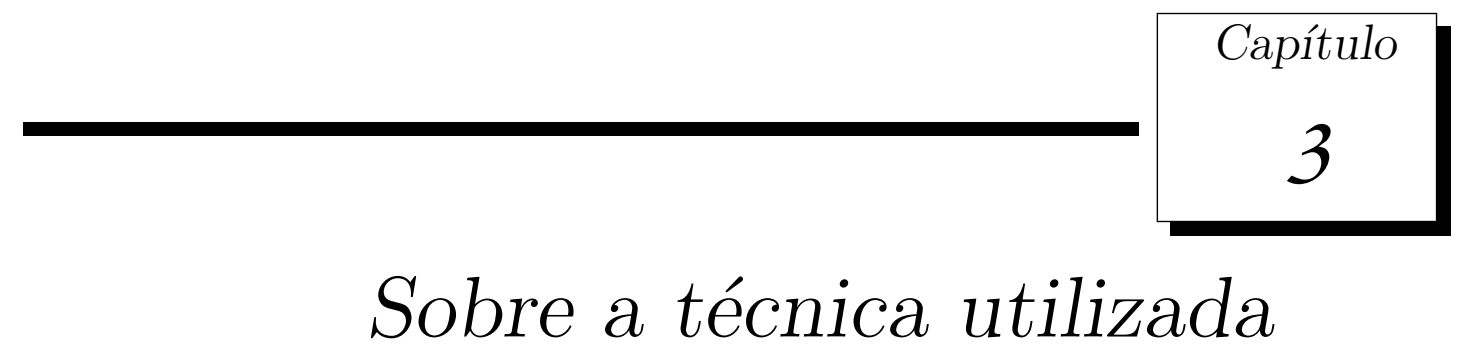

Nesse capítulo, destacamos outros trabalhos que investigam o retrato de fase de campos de vetores polinomiais planos utilizando a mesma técnica descrita nessa dissertação. São eles:

1. A. Cima, J. Llibre (1990) - Algebraic and topological classification of the homogeneous cubic vector fields in the plane.

2. J. Chavarriga, B. Garcia, J. Llibre, J.S. Pérez Del Rio e J.A. Rodrigues (2005) Polynomial first integrals of quadratic vector fields.

3. B. Garcia, J. Llibre e J.S. Pérez Del Rio (2005) - Phase portraits of the quadratic vector fields with a polynomial first integral.

4. L.Cairó, J. Llibre (2007) - Phase portraits of cubic polynomial vector fields of LotkaVolterra type having a rational first integral of degree 2.

5. C. A. Buzzi, D.J. Tonon (2009) - Quadratic planar systems with two parallel invariant straight lines.

6. J. Llibre, R.D.S. Oliveira (2009) - Phase portraits of quadratic polynomial vector fields having a rational first integral of degree 3.

7. A. Mahdi (2008) - Integrable polynomial differential systems and their perturbations.

Em 1900, Hilbert participou do Congresso Internacional de Matemática em Paris, onde propôs 23 grandes problemas para serem investigados no próximo século. Sobre a segunda parte do décimo sexto problema, ele disse: [12, 15] 
Gostaria de apresentar uma questão que, ao que me parece, pode ser resolvida pelo mesmo método de variação contínua de coeficientes, e cuja resposta é de correspondente valor para a topologia de familias de curvas definidas por equações diferenciais. Esta é a questão quanto ao número máximo e a posição de "Poincaré's boundary cycles"(ciclos limites), para uma equação diferencial de primeira ordem e de grau d da forma:

$$
\frac{d y}{d x}=\frac{X}{Y}
$$

onde $X$ e $Y$ são funções integrais racionais de grau d em $x$ e $y$.

Segundo Smale, este é um dos problemas mais desafiadores propostos por Hilbert. Um número muito grande de matemáticos tem procurado a solução deste problema, mas esta, todavia, está muito longe de ser obtida, mesmo para o caso $d=2$. Também a questão da finitude dos ciclos limites está longe de alcançar seu desenvolvimento definitivo. Em 1923, Dulac publicou o artigo Sur les cycles limites onde "provou"a finitude dos ciclos limites para um dado sistema planar polinomial. Mais tarde, a sua prova foi concluída por Il'yashenko [15] e Écalle [12]. O teorema de finitude não diz nada sobre a existência do limitante superior uniforme para os ciclos limites em função do grau. Em outras palavras, a resposta para a questão abaixo não é conhecida:

Considere

$$
\frac{d x}{d t}=P(x, y), \quad \frac{d y}{d t}=Q(x, y)
$$

onde $P$ e $Q$ são polinomiais. Existe um limitante $K$ para o número de ciclos limites da forma $K \leq d^{q}$, onde $d$ é o máximo dos graus de $P$ e $Q$ ?

Devido à dificuldade em resolver esta questão, pesquisadores passaram a estudar problemas menos desafiantes que poderiam ser resolvidos, como por exemplo, classificar os retratos de fase globais dos sistemas (3.1), onde $d=1,2$ e 3 e, desta maneira, encontrar o número de ciclos limites existente para cada valor de $d$.

A seguir, descreveremos alguns destes trabalhos e a técnica utilizada em cada um para a obtenção dos retratos de fase.

Em 1990, Cima e Llibre, em [8] estudaram o sistema (3.1), onde $P$ e $Q$ são polinômios homogêneos de grau 3.

Neste trabalho, inicialmente é obtida a classificação algébrica de tais sistemas, isto é, dado um sistema arbitrário do tipo (3.1), é possível saber a qual classe de equivalência ele pertence por meio de suas características algébricas. 
Em seguida, é feito um estudo dos retratos de fase desses sistemas, onde $P$ e $Q$ são polinômios homogêneos de grau $n$ e, $P$ e $Q$ são coprimos, isto é, não têm fator comum. É provado também que esses resultados podem ser aplicados para determinar genericamente o comportamento no infinito dos sistemas (3.1), não necessariamente homogêneos. Por último, foram obtidos todos os possíveis retratos de fase dos campos vetoriais da forma (3.1), com $P$ e $Q$ polinômios homogêneos onde $\operatorname{grau}(P)=\operatorname{grau}(Q)=3$.

Dizemos que um campo vetorial $X$, ou equivalentemente que o sistema (3.1), é integrável em um conjunto aberto $U$ de $\mathbb{R}^{2}$, se ele tem uma integral primeira.

A busca por integrais primeiras é uma ferramenta clássica para descrever o retrato de fase de um sistema diferencial bidimensional. Por este motivo, muitos pesquisadores passaram a estudar os campos vetoriais integráveis.

Em 2006, Chavarriga e outros, em [7], classificaram algebricamente os sistemas quadráticos com uma integral primeira polinomial e forneceram formas normais explícitas para tais sistemas e suas integrais primeiras polinomiais. A técnica utilizada para a obtenção de tais formas normais e suas integrais primeiras é baseada em mudança de coordenadas afim e na manipulação algébrica dos sistemas.

A partir da classificação algébrica fornecida em [7], em 2006, Garcia, Llibre e Pérez Del Rio, em [13] classificaram topologicamente os retratos de fase de todos os sistemas diferenciais polinomiais quadráticos com integral primeira polinomial.

A técnica utilizada para encontrar os retratos de fase dos sistemas quadráticos com integral primeira polinomial é a mesma utilizada por Cairó e Llibre em [5], e descrita nesta dissertação.

A análise dos pontos singulares finitos é feita com base no estudo realizado em [7], o qual estabelece condições sobre os auto-valores da matriz Jacobiana de um sistema quadrático com integral primeira polinomial.

O estudo dos pontos singulares infinitos é feito através da compactificação de Poincaré.

Após o estudo dos pontos singulares finitos e infinitos, são obtidos os retratos de fase no disco de Poincaré.

Se $H(x, y)$ é um polinômio de grau $n+1$, então o sistema

$$
\left\{\begin{array}{l}
\dot{x}=-\frac{\partial H}{\partial y} \\
\dot{y}=\frac{\partial H}{\partial x}
\end{array}\right.
$$


é chamado sistema Hamiltoniano de grau $n$. No mesmo artigo, também é provado que todos os retratos de fase encontrados são realizáveis por sistemas Hamiltonianos de grau 2 , e que, todos os retratos de fase dos sistemas diferenciais polinomiais lineares com uma integral primeira polinomial, são também realizáveis por sistemas Hamiltonianos de grau 1. Então, a seguinte questão aparece em aberto: Todos os retratos de fase de sistemas diferenciais polinomiais de grau $n$ com uma integral primeira polinomial, são realizáveis por sistemas Hamiltonianos de grau $n$ ?

Dados $P$ e $Q$ dois polinômios reais nas variáveis $x$ e $y$, dizemos que $X=(P, Q): \mathbb{R}^{2} \rightarrow$ $\mathbb{R}^{2}$ é um campo vetorial polinomial cúbico se o máximo dos graus dos polinômios $P$ e $Q$ é 3. Dizemos que um campo de vetores polinomial cúbico é do tipo Lotka-Volterra, se $x$ é um fator de $P$, e $y$ um fator de $Q$.

Os sistemas de Lotka-Volterra normalmente modelam a evolução temporal do conflito de espécies em biologia. Existem muitos outros fenômenos naturais modelados por sistemas de Lotka-Volterra, tais como acoplamento de ondas laser em Física e a evolução de elétrons, íons e espécies neutras no plasma.

Em 2007, Cairó e Llibre classificaram todos os retratos de fase globais dos campos vetoriais polinomiais cúbicos do tipo Lotka-Volterra com uma integral primeira racional de grau 2.

Em busca dos retratos de fase de tais sistemas, inicialmente é mostrado que qualquer campo de vetores polinomial cúbico do tipo Lotka-Volterra $(P, Q)$ com uma integral primeira racional de grau 2, não polinomial, pode ser escrito na forma: $P=x\left(a+b x+d x^{2}-\right.$ $\left.e y^{2}\right), Q=y\left(-a-c y+d x^{2}-e y^{2}\right)$ com integral primeira: $H=\frac{a+b x+c y+d x^{2}+e y^{2}}{x y}$.

Dessa forma, é obtido um campo vetorial com 5 parâmetros. Com o intuito de reduzir esse número de parâmetros, é aplicada uma mudança de coordenadas lineares ao campo acima. Dessa mudança de coordenadas, resultam 16 formas normais, sendo que cada uma delas tem apenas 2 parâmetros. Sabendo agora que qualquer campo de vetores polinomial cúbico $(P, Q)$ do tipo Lotka-Volterra, com uma integral primeira de grau 2 e com $P$ e $Q$ coprimos pode ser escrito como um dos 16 campos vetoriais obtidos, o último passo é encontrar os retratos de fase de cada um desses 16 campos vetoriais. Novamente, a técnica utilizada para obter os retratos de fase é a utilizada por Cairó e Llibre em [5], e descrita nesta dissertação. Primeiramente, é feita a classificação dos pontos singulares infinitos a partir da compactificação de Poincaré. Em seguida, é feita a classificação dos pontos 
singulares finitos, por último, o retrato de fase no disco de Poincaré.

Observe que, se $X$ é um campo de vetores polinomial do tipo Lotka-Volterra, então, o eixo $x$ e o eixo $y$ são invariantes pelo fluxo de $X$. Naturalmente, existem muitos tipos de sistemas que têm duas retas invariantes. A classe de sistemas mais conhecida provavelmente é a classe dos sistemas de Lotka-Volterra. É fácil ver que, se um campo vetorial tem duas retas transversais invariantes então uma mudança linear de coordenadas transforma este campo vetorial em um do tipo Lotka-Volterra.

Surge então, o interesse em estudar os sistemas com duas retas paralelas invariantes.

Em [4], Buzzi e Tonon classificaram os retratos de fase globais no disco de Poincaré dos campos de vetores polinomiais quadráticos $X=(P, Q)$ com duas retas paralelas invariantes. Assumindo, sem perda de generalidade, que essas retas invariantes são $x=1$ e $x=-1, X$ pode ser escrito na seguinte forma: $X=\left((x+1)(x-1), a+b x+c y+d x^{2}+e x y+f y^{2}\right)$. Observe que esse sistema tem 6 parâmetros independentes. Após uma mudança de coordenadas lineares, é possível reduzir o estudo desse sistema ao estudo de 5 formas normais, tomando apenas parâmetros essenciais. Finalmente, o problema se resume a encontrar os retratos de fase de cada um desses cinco campos. Novamente, a técnica utilizada para obtenção dos retratos de fase é a utilizada por Cairó e Llibre em [5] e descrita nesta dissertação.

Em 2008, Llibre e Oliveira classificaram todos os retratos de fase globais dos campos vetoriais quadráticos $X=(P, Q)$ com uma integral primeira racional de grau 3. Inicialmente, eles definiram $P$ e $Q$, onde

$$
P=-\frac{\partial H}{\partial y} H_{2}^{2}, Q=\frac{\partial H}{\partial x} H_{2}^{2} \text { e } H=\frac{H_{1}}{H_{2}}=\frac{\sum_{i+j=0}^{3} e_{i j} x^{i} y^{j}}{\sum_{i+j=0}^{3} a_{i j} x^{i} y^{j}}
$$

Como $\operatorname{grau}(P, Q) \leq 5$ para se obter todos os sistemas quadráticos com integral primeira de grau 3, seguiram dois caminhos.

Primeiramente, anularam os coeficientes de grau 3, 4 e 5 de $P$ e $Q$ e mostraram que tal sistema não apresenta solução para $H$ integral racional cúbica.

Em seguida, consideraram o caso em que $P$ e $Q$ têm um fator comum $R$ de grau 1,2 ou 3 , e o máximo dos graus de $\frac{P}{R}$ e $\frac{Q}{R}$ é 2 .

Para encontrar todos os sistemas satisfazendo essas condições, o primeiro passo foi reduzir o número de variáveis do sistema. Sem perda de generalidade, assumindo que o 
numerador $H_{1}$ de $H$ é um polinômio de grau 3 nas variáveis $x$ e $y$, obtiveram que $H_{1}$ ou é um polinômio cúbico irredútivel ou é dado pelo produto de uma cônica e uma reta. Dessa forma, o estudo foi reduzido ao estudo de 13 casos. Para cada um dos 13 casos encontraram $P=-\frac{\partial H}{\partial y} H_{2}^{2}$ e $Q=\frac{\partial H}{\partial x} H_{2}^{2}$ e $R$ tal que

$$
\begin{aligned}
& P-R(x, y) \bar{P}(x, y)=0 \\
& Q-R(x, y) \bar{Q}(x, y)=0
\end{aligned}
$$

onde

$$
\begin{gathered}
\bar{P}(x, y)=c_{00}+c_{10} x+c_{01} y+c_{20} x^{2}+c_{11} x y+c_{02} y^{2}, \\
\bar{Q}(x, y)=d_{00}+d_{10} x+d_{01} y+d_{20} x^{2}+d_{11} x y+d_{02} y^{2}, \\
\bar{R}(x, y)=b_{00}+b_{10} x+b_{01} y+b_{20} x^{2}+b_{11} x y+b_{02} y^{2}+b_{30} x^{3}+b_{21} x^{2} y+b_{12} x y^{2}+b_{03} y^{3},
\end{gathered}
$$

Esses sistemas foram resolvidos com a ajuda do Mathematica.

Em seguida, utilizando mudança de coordenadas lineares, foram encontradas as formas normais para o campo $X$ e para a integral primeira $H$.

Finalmente, é feito um estudo local dos pontos singulares finitos e infinitos para cada uma das formas normais encontradas. A partir desse estudo, foram obtidos os retratos de fase globais no disco de Poincaré.

Seguindo investigação semelhante, Mahdi, em [19], classifica os retratos de fase dos sistemas cúbicos com uma integral primeira racional de grau dois que possuem um centro. Inicialmente, é mostrado que, qualquer sistema cúbico da forma (3.1) com um centro na origem e com uma integral primeira racional $H$ de grau dois, pode ser escrito em uma das duas formas abaixo:

$$
\left\{\begin{array}{l}
\frac{d x}{d t}=y\left(-d+a^{2} d-2 a d x-b x^{2}+d x^{2}\right) \\
\frac{d y}{d t}=b x-a^{2} x+a b x^{2}-a d y^{2}-b x y^{2}+d x y^{2}
\end{array}\right.
$$

com uma integral primeira

$$
H=\frac{b x^{2}+d y^{2}}{-1+a^{2}-2 a x+x^{2}+y^{2}}
$$

$\mathrm{Ou}$

$$
\left\{\begin{array}{l}
\frac{d x}{d t}=\left(1-a^{2}\right)(x+2 d y)+2 a x^{2}+4 d a x y-x^{3}+2(b-d) x^{2} y+x y^{2} \\
\frac{d y}{d t}=\left(a^{2}-1\right)(2 b x+y)-2 a b x^{2}+2 a d y^{2}-x^{2} y+2(b-d) x y^{2}+y^{3}
\end{array}\right.
$$


com uma integral primeira

$$
H=\frac{b x^{2}+x y+d y^{2}}{-1+a^{2}-2 a x+x^{2}+y^{2}}
$$

onde $a \in[0,1)$.

Enfim, são obtidos os retratos de fase dos sistemas acima. A técnica utilizada para encontrar os retratos de fase é a utilizada em [5].

Quase todos os trabalhos, acima descritos, deram origem a um segundo trabalho sobre o estudo dos ciclos limites que bifurcam de um centro, quando perturbado dentro da classe à qual os campos de vetores polinomiais pertencem. 



\section{Referências Bibliográficas}

[1] A. F. Andreev. Investigation of the behaviour of the integral curves of a system of two differential equations in the neighborhood of a singular point. Translation of Amer. Math. Soc., 8, (1958), 183-207.

[2] A. A. Andronov, E.A. Leontovich, I.I. Gordon and A.G. Maier. Qualitative theory of second order dynamic systems. Jonh Willey \& Sons, New YorkToronto, 1973.

[3] J. C. Artés, F. Dumortier, C. Herssens, J. Llibre, and P. De Maesschalck. Computer program $\mathrm{P}_{4}$ to study phase portraits of plane polynomial diferential equation. http://mat.uab.es/ artes/p4/p4.htm, 2005.

[4] C. A. Buzzi, D.J. Tonon. Quadratic Planar systems with two parallel invariant straight lines. Qualitative Theory of Dynamical Systems 7, (2009) $295-316$.

[5] L. Cairó, J. Llibre. Phase portraits of quadratic polynomial vector fields having a rational first integral of degree 2. Nonlinear Anal., Ser. A: Theory and Methods 67, (2007), 327-348.

[6] L. Cairó, J.Llibre. Phase portraits of cubic polynomial vector fields of LotkaVolterra type having a rational first integral of degree 2. Journal of Physics A: Mathematical and Theoretical 40 (2007), 6329-6348.

[7] J. Chavarriga, B.Garcia, J. Llibre, J.S. Pérez Del Rio and J.A. Rodrigues. Polynomial first integrals of quadratic vector fields. J. Differential Equations 230 (2006), 393-421. 
[8] A. Cima, J. Llibre. Algebraic and topological classification of the homogeneous cubic vector fields in the plane. J. Math, Anal. Appl. 147, (1990), $420-448$.

[9] G. Darboux. Mémoire sur les équations différentielles algébriques du premier ordre et du premier degré. Bull. Sci. Math. 2 (1978), 60-96; 123-144; 151200.

[10] F. Dumortier. Singularities of vector fields. Monografias de Matemática, 32, IMPA/ CNPq, 1978.

[11] F. Dumortier. J. Llibre and J.C. Artés. Qualitative theory of planar differential systems. UniversiText, Springer-Verlag, 2006.

[12] J. Écalle. Introduction aux fonctions analysables et preuve constructive de la conjecture de Dulac. Actualités Math. Hermann, Paris, 1992.

[13] B. Garcia, J. Llibre and J.S.Pérez Del Rio. Phase portraits of the quadratic vector fields with a polynomial first integral. Rend. Circ. Mat. Palermo (2) 55 (2006), 420-440.

[14] P. Hartman. Ordinary differential equations. Reprint second edition. Birkhäuser, Boston, Mass, 1982.

[15] Y. Il'Yashenko. Finiteness theorems for limit cycles. Trans. of Math. Monographs. Amer. Math. Soc., vol. 94, 1991.

[16] J. P. Jouanolou. Équations de Pfaff algébriques. Lectures Notes in Math., vol. 708, Springer, New York, 1979.

[17] J. Llibre, R.D.S. Oliveira. Phase portraits of quadratic polynomial vector fields having a rational first integral of degree 3. Nonlinear Analysis 70 (2009), 3549-3560.

[18] J. Llibre. Integrability of polynomial differential systems. Handbook of differential equations, Ordinary Differential Equations, Elsevier, vol.1, (2004), $437-533$. 
[19] A. Mahdi. Integrable polynomial differential systems and their perturbations. Ph Thesis, UAB, (2008).

[20] J. Sotomayor. Curvas definidas por equações diferenciais no plano. $13^{0}$ Colóquio Brasileiro de Matemática, IMPA, (1981).

[21] J. Sotomayor and R. Paterlini. Quadratic vector fields with finitely many periodics orbits. Springer Lectures Notes in Math. 1007 (1983), 753-766.

[22] Ye Yan Quian and others. Theory of limit cycles. Trans. of Mathematical Monographs, vol. 66, Amer. Math. Soc., Providence, RI, second edition, (1984). 



\section{Índice Remissivo}

Blow up, 11

direcional, 15

polar, 11

Compactificação de Poincaré, 24

Conjunto invariante, 3

Curva algébrica invariante, 4

Disco de Poincaré, 29

Equivalência topológica, 24

Esfera de Poincaré, 23

Fluxo, 3

Integral primeira, 5

racional de grau 2, 6

Ponto singular, 6

degenerado, 7

finito, 28

hiperbólico, 7

infinito, 28

nilpotente, 8

não degenerado, 7

semi-hiperbólico, 7

linearmente nulo, 9

Retrato de fase, 6
Sistema integrável, 101

Trajetória maximal, 3 